\title{
GENETIC MODIFICATION OF SHORT ROTATION POPLAR BIOMASS FEEDSTOCK FOR EFFICIENT CONVERSION TO ETHANOL
}

\author{
Final Report
}

Ronald J. Dinus

Environmental Sciences Division

June 2000

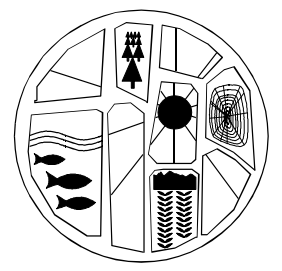




\section{GENETIC MODIFICATION OF SHORT ROTATION POPLAR BIOMASS FEEDSTOCK FOR EFFICIENT CONVERSION TO ETHANOL}

Final Report

Report Prepared by

Dr. Ronald J. Dinus, Consultant

2490 Goshen Road

Bellingham, WA 98226-9556

Telephone/FAX: 360-966-4027

email:dinus@telcomplus.net

Environmental Sciences Division

Date published: June 2000

Research supported by

U.S. Department of Energy

Office of Fuels Development

Activity No. EB 5203000

Prepared for

Bioenergy Feedstock Development Program

Environmental Sciences Division

Oak Ridge National Laboratory

managed by

University of Tennessee-Battelle, LLC

for the

U.S. Department of Energy

under contract DE-AC05-00OR22725 


\section{CONTENTS}

Page

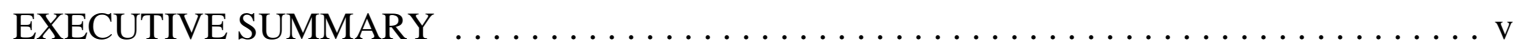

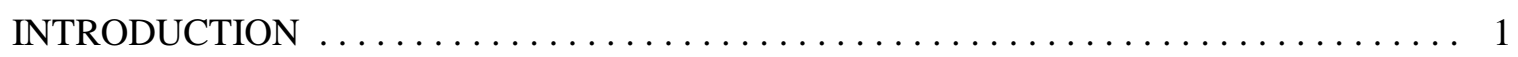

FEEDSTOCK QUALITY AND ETHANOL CONVERSION PROCESSES $\ldots \ldots \ldots \ldots \ldots$.

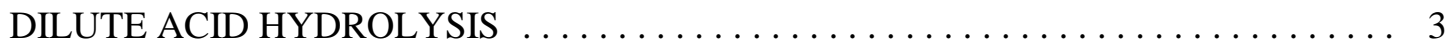

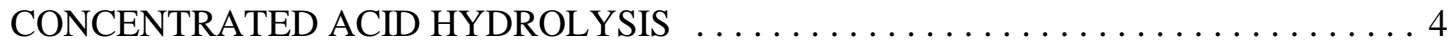

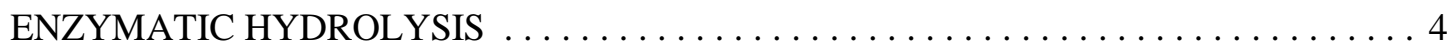

FERMENTATION OF C5 SUGARS TO ETHANOL $\ldots \ldots \ldots \ldots \ldots \ldots \ldots \ldots \ldots$

KNOWN IMPACTS OF CHANGES IN FEEDSTOCK QUALITY $\ldots \ldots \ldots \ldots \ldots \ldots . \ldots$

CHARACTERISTICS OF COMMERCIALLY AVAILABLE FEEDSTOCKS $\ldots \ldots \ldots 6$

DESIRABLE CHANGES IN FEEDSTOCK QUALITY $\ldots \ldots \ldots \ldots \ldots \ldots \ldots \ldots$

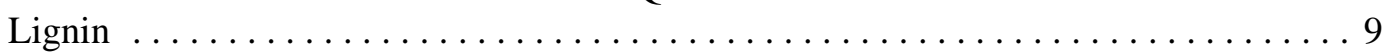

Cellulose ...................................... 10

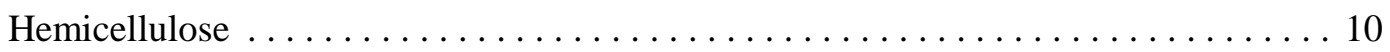

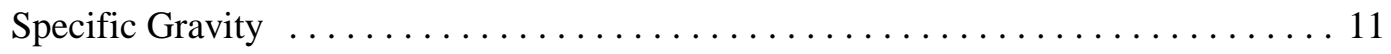

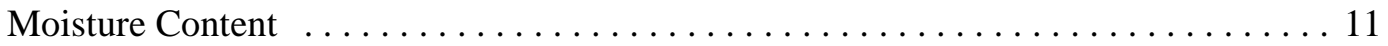

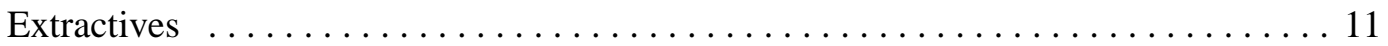

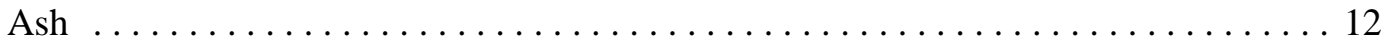

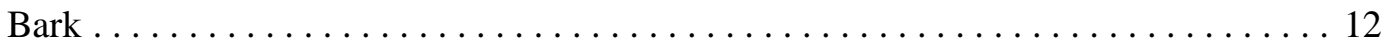

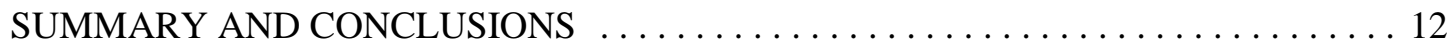

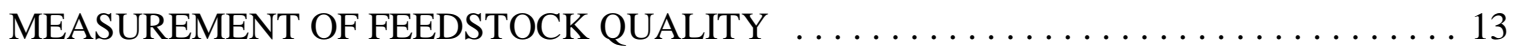

LIGNIN, CELLULOSE, AND HEMICELLULOSE CONTENTS $\ldots \ldots \ldots \ldots \ldots \ldots \ldots$

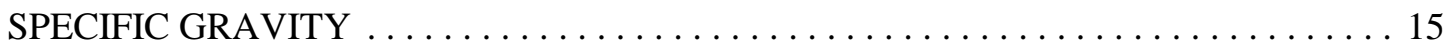

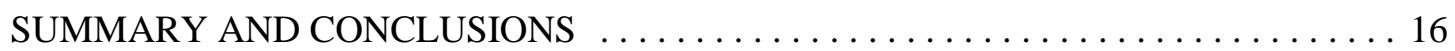

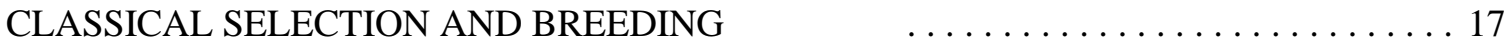

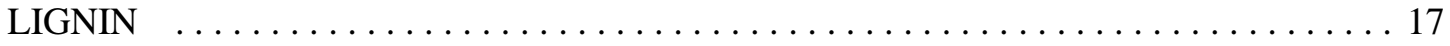

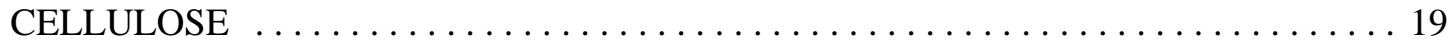

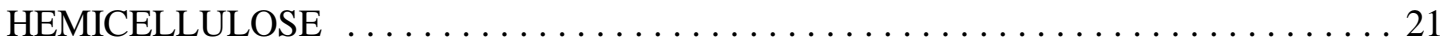

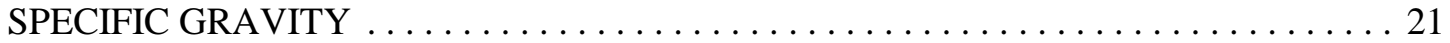

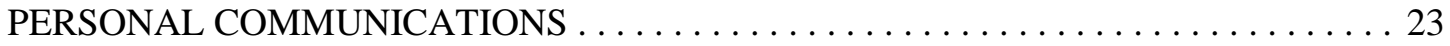

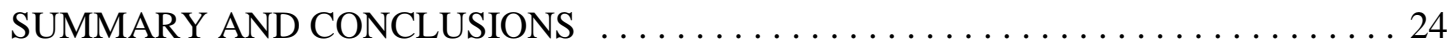

GENETIC MARKERS AND MAPS . . . . . . . . . . . . . . . . . . . . . 27

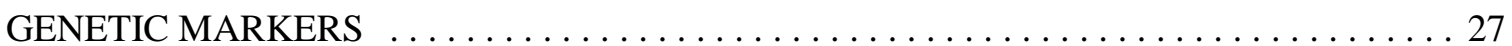

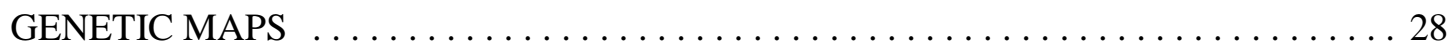




\section{CONTENTS (cotinued)}

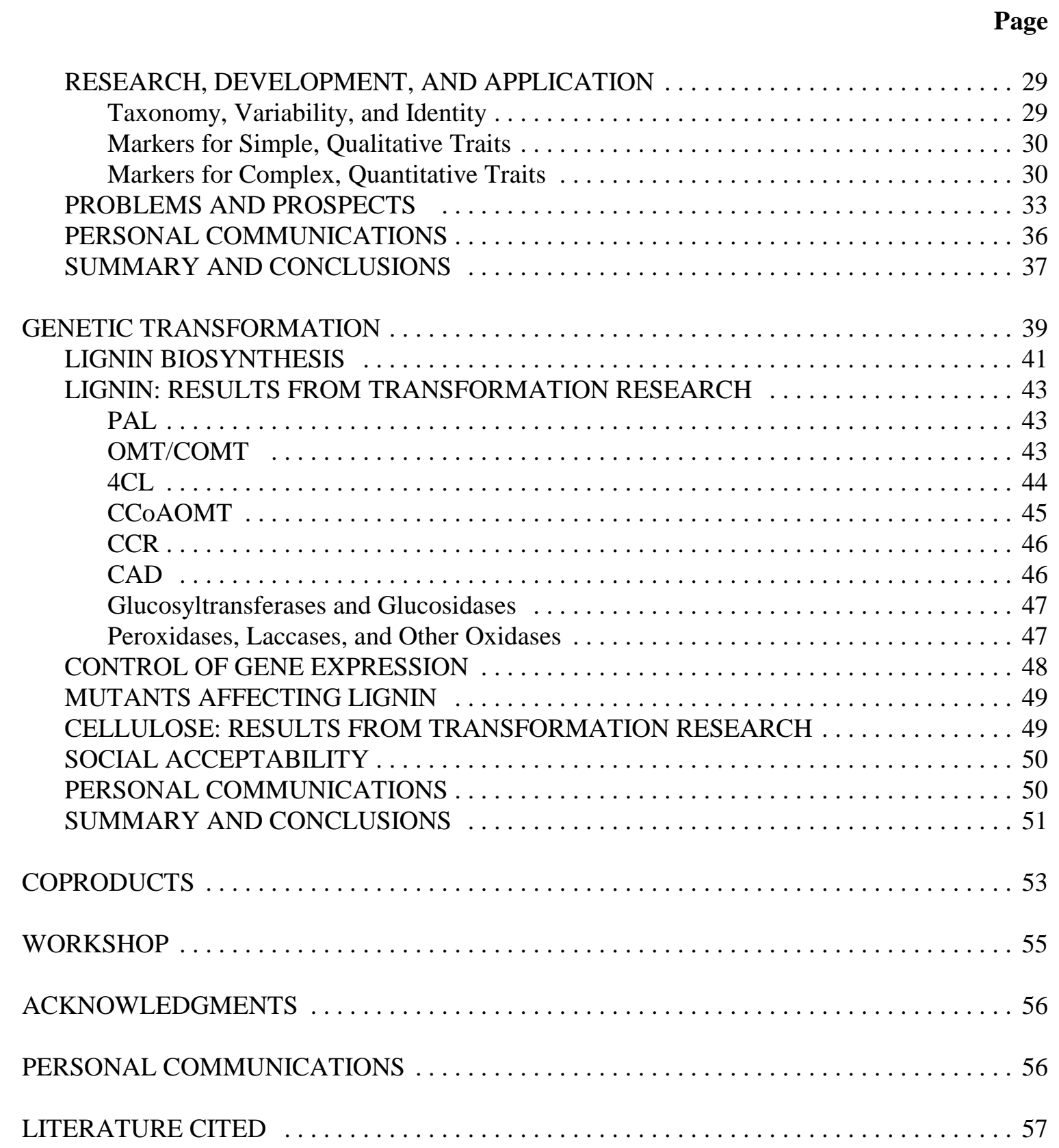




\section{EXECUTIVE SUMMARY}

Poplar breeders in the United States have focused on raising adaptability, growth rates, and pest/stress resistance. Significant increases have been achieved via classical selection and breeding along with intensified cultural practices. Efficiencies have also been gained in harvesting and handling. Considering the aforementioned improvements in productivity, the time seems optimal for choosing feedstock qualities to modify and for undertaking the necessary research and development. Indeed, shortened rotations resulting from past research mean that opportunities for modifying feedstock qualities can be exploited more easily. Many poplars are propagated clonally, thereby permitting rapid, inexpensive propagation of valuable variants. Thus, poplars seem an ideal venue for testing and applying techniques to improve feedstock quality, e.g., reduced lignin content.

Opportunities for manipulating feedstock quality have long been recognized but have largely gone unrealized because of uncertainties over which traits to modify for what process. Quality changes can affect process efficiency in numerous ways, but reliable information concerning impacts is sparse. Process specialists must cooperate with breeders and growers to identify leverage points and quantify impacts such that economic weights can be combined with genetic information to plan and execute effective modification strategies. The outcome could be poplar varieties better suited to process requirements, and more likely to reduce ethanol production costs than varieties with only increased growth.

Measurement methods that are accurate, rapid, and inexpensive, and that rely on small, nondestructively collected samples are needed for genetic modification of feedstock quality. Recent advances in instrumentation, methods, and economy have been significant and are opening the way to efficient modification of wood chemical and physical properties and, perhaps, even to specifying processing conditions for different feedstocks.

Classical selection and breeding can be used to reduce lignin content, albeit slowly. Raising cellulose content is more difficult, given its weak genetic control and negative relationship to growth. Both might be altered more efficiently via interspecific hybridization to expand ranges of genetic variation and clonal propagation of outstanding offspring. As an alternative, breeding might better focus on specific gravity or dry wood production, a highly heritable trait likely to have indirect but favorable effects on lignin and cellulose contents. Positive outcomes would accrue in production (e.g., harvesting and transportation) as well as in processing (e.g., more cellulose and less lignin). This tack seems particularly useful in that products would be attractive to a variety of customers.

Developments in molecular biology have led to detection of genetic markers associated with useful traits. The pace of development has been rapid, and further improvements in utility and economy can be expected, if only from undertakings such as the Human Genome Project. In theory, markers can be used to screen breeding materials and/or offspring from crosses among selected parents at early ages. Only those with desired markers would be tested in the field or moved into production, and years of testing large numbers of individuals with uncertain value could be avoided. Markers are available for identifying poplar genotypes, a considerable aid to 
managing breeding programs, and for use in research on poplar taxonomy, hybridization, and genetic variation. Such markers may soon be extended to early determinations of gender and monitoring potential for gene flow from transgenic trees. As concerns selection, markers available to date have proven useful only for qualitative traits, e.g., disease resistance, or that are highly heritable, difficult and/or expensive to measure, and of high value. Most traits affecting feedstock quality, however, are quantitatively, rather than qualitatively, inherited, and useful markers have proven difficult to identify. Also of concern is unreliability over the diversity of populations and families used in breeding, tree ages used even in short-rotation forestry, and environments encountered in commercial programs. In consequence, most investigators argue that marker-aided selection is not ready for application, and that investigations should focus on identifying and dissecting quantitative trait loci. Results could one day allow describing quantitative traits in terms of gene numbers, individual gene effects, and modes of action as well as discerning the basis of heterosis. In addition, candidate genes thus identified might be used to investigate gene function and perhaps transform individuals with other desirable traits.

Genetic transformation has been advocated as a desirable and feasible means of improving wood physical and chemical properties. A host of U.S. and offshore investigators are pursuing research on this front to good effect, particularly as concerns lignin and cellulose contents. Transgenic poplar trees with significantly reduced lignin and increased cellulose contents have been produced and are being evaluated under controlled field conditions. Transformants are growing faster than nonmodified controls, and are morphologically and anatomically normal.
Commercialization is only a matter of time, perhaps 5-10 years.

Several concerns could impede commercialization. Perhaps the major obstacle is public concern that transgenic trees will spread so-called foreign genes to natural populations. One means of minimizing, if not avoiding, this risk is to render transformants sexually sterile. Research has progressed rapidly, and genetic constructs should be ready for use in poplars within 5-10 years. Other benefits may also accrue; eliminating reproductive structures could channel more energy, water, and nutrients into wood production. Concern also exists about mechanisms for commercialization. Developing and applying transformation technology is expensive. Licensing fees are also a worrisome issue, since organizations engaged in transformation research generally patent their creations. Timely technology transfer may require fostering growth of independent laboratories that license genetic constructs, effect transformation, and propagate transformants for pools of breeders, nurseries, and/or growers.

Coproducts clearly could increase feedstock values, and trees could be modified to produce a variety of useful materials. Even so, genetically modifying poplars to yield valuable products, e.g., pharmaceuticals, while also striving to increase growth and feedstock quality would complicate matters and raise costs. The more that coproduct characteristics diverge from those optimized for ethanol conversion, the greater the difficulty and expense. Some coproducts, however, are compatible with ethanol production, e.g., high value solid wood products such as molding and trim, veneers, and furniture parts. Accordingly, this seems the best approach to development of poplar coproducts over the near term.

Balanced support of additional research, development, and technology 
transfer is essential to ensure that dividends from the aforementioned advances are realized in a reasonable time frame. Poplar feedstock bred for rapid growth and high dry wood substance production and genetically transformed to have lower lignin and heightened cellulose contents stand to have tremendous impact on ethanol production efficiency.

\section{RECOMMENDATIONS FOR RESEARCH, DEVELOPMENT, AND APPLICATION}

\section{Ethanol Conversion Processes}

- The impacts of changes in poplar feedstock quality on process efficiency must be clarified. Analyses to date have focused largely on costs of growing, harvesting, handling, transportation, and storage. Few have addressed changes in chemical and/or physical properties, which can affect process efficiency in numerous ways and at a variety of points in the process. Breeders and growers must know which traits to modify and how to modify them for which process, as well as how they will be compensated before investing time and dollars to effect change. Definitive, real-life trials with feedstocks of differing qualities are needed to determine effects of changes on each of the many steps in individual processes, and to quantify the economic value of such changes. Research on these fronts is of the highest priority.

\section{Feedstock Quality Measurement}

- $\quad$ Efficient genetic modification requires that feedstock characteristics be measured accurately, inexpensively, and nondestructively. Sponsoring further research on and development of efficient instrumentation and methods should bring yet new capabilities, heightened availability, and further cost reductions. Fostering establishment and growth of independent testing laboratories also seems a worthy goal.

\section{Classical Selection and Breeding}

- $\quad$ Poplar breeding and growing programs seem sufficiently mature to add objectives targeted at improving feedstock quality. Breeding efforts in future should be designed to reduce costs and raise efficiencies at all significant leverage points in the production and processing system. Accomplishing this requires selection indices that combine genetic data with economic weights associated with impacts on process leverage points. Stimulating collaborative research, development, and application activities among breeding and process specialists is a critical need and demands immediate attention.

- Genetic information about important quality traits, as inferred above, is adequate for the short term. For the long term, however, more and better information is needed. More reliable data on extent of genetic variation and control would be useful. Of particular concern, however, is data on relationships among quality traits and growth, correlations across ages, and genotype $\mathrm{X}$ environment interactions. Results are prerequisite to development of the selection indices described above, and the associated research is therefore of high priority.

- Interspecific hybridization offers one means for increasing the generally narrow ranges of genetic variation in most quality traits, especially lignin and cellulose contents. Future 
breeding efficacy demands that such techniques be evaluated. Poplar species are quite numerous, and many can be cross bred with relative ease.

Research on expanding variability and acquiring heterosis, therefore, holds considerable promise and commands at least moderate priority.

- Data on genetic variation and control of hemicellulose content in poplars are especially scarce. Recent reports on Eucalyptus species suggest significant variation and strong control. Research on genetic parameters therefore seems warranted, given the potential for increasing carbohydrate content and lessening hemicellulose-lignin linkages.

- Modifying quality traits in addition to lignin, cellulose, and specific gravity could have merit. Reduced moisture content could have effects similar to or complementary to high specific gravity. Reducing extractives, ash, and bark amounts, naturally low in poplars, could have favorable, though incremental, impacts. Information is limited on the extent to and means by which such traits can be modified. Research to acquire needed genetic data seems warranted, but is best pursued within investigations of the most influential traits.

\section{Genetic Markers and Maps}

- Genetic marker technologies, even now, are being used to identify poplar genotypes in classical selection and breeding programs. Continued development of gender determination markers would do much to reduce costs and increase efficiency. Extension to monitoring potential gene flow from transgenic trees to natural populations would constitute a valuable contribution to public acceptance of genetic transformation and, therefore, deserves high priority.

- The fact that genetic markers currently seem useful only within families and for a limited suite of traits greatly restricts application. Costs of both development and application are high and could drain resources from classical selection and breeding. Meaningful use awaits development of markers that are generalizable across species; populations and families; environments; ages; and generations. To best achieve these ends, future research should be aimed at identifying and analyzing quantitative trait loci, especially those related to cell wall biosynthesis. Quantitative trait loci analyses for trait dissection and candidate gene analysis and expression, as well as mechanisms underlying heterosis, also deserve more attention. Future research in these areas should be pursued with diverse genetic backgrounds, large sample sizes, and clonal replication across multiple sites.

- Attention should also be given to developing consensus genetic maps such that markers and other genetic information can be transferred freely across genetic backgrounds, including both poplars and other well-known model species.

- $\quad$ Research to verify and validate marker applications should be given high priority and should involve paired comparison trials, i.e., trees chosen on the basis of marker technologies versus trees obtained via classical selection and breeding, spanning all or most of a rotation and involving several environments. Such trials should give particular attention to establishing the 
economic feasibility of marker applications.

- Advances in marker technology are likely to be accompanied by formation of not only more but also more sophisticated university/industrial cooperatives and private service laboratories. Fostering growth of such enterprises would facilitate application of any marker technologies that prove useful to poplar breeding.

\section{Genetic Transformation}

- Methods for poplar transformation are available, and a number of genes have been inserted and expressed in various species and hybrids. Poplars actually are considered models for such research, given the ease with which many can be manipulated and regenerated from cell and tissue cultures. Improvements in transformation efficiency and extension to wider arrays of genotypes, however, remain important research needs.

- Genetic transformation has been used successfully to reduce lignin and increase cellulose contents. To date, most genetic interventions in lignin biosynthesis have been done at the level of precursor synthesis. Support of this largely successful research should be amplified and extended to include that on the physiology, biochemistry, and genetics of precursor transport, storage, deposition, and polymerization. Intervention at late points in lignin biosynthesis could have less effect on other metabolic processes than precursor manipulation. Given the utility of low lignin content, accelerated research on such topics seems essential.
- Information on cellulose biosynthesis is less abundant than that on lignin. Accordingly, arguments given above for continued support of lignin research also apply to that on cellulose. Modifying both lignin and cellulose contents should have significantly beneficial effects on ethanol production efficiency.

- Expression of transgenes in appropriate tissues and developmental stages is critical to successful genetic transformation. Much transformation research done to date has been conducted with constitutive promoters. Results have been spectacular, but xylem specific promoters are needed if outcomes are to be practical and stable. Research on identifying, isolating, and applying native promoters warrants acceleration.

- Despite initial successes, the possibility remains that modification of lignin/cellulose biosynthesis via genetic transformation will have detrimental effects on survival, growth, pest resistance, and/or other tree functions over the length of a rotation. Provision for research on stability and delayed responses is therefore important.

- $\quad$ Potential spread of so-called foreign genes from transgenic trees into natural populations is a major worry to the public. Minimizing this risk seems best accomplished by inducing sexual sterility in transformed trees. Research is this area has been productive, but continued support is critical for eventual realization of the substantial benefits resulting from genetic transformation.

- Mechanisms for commercialization of transformed trees also merit attention. 
Few breeders; nurseries; and growers, including some large but research-shy companies, have the financial wherewithal to license genetic constructs or plant materials, transform their own breeding materials, and/or engage in joint ventures with transformation research organizations. Accordingly, developing and stimulating means for timely technology transfer may be necessary.

\section{Coproducts}

- Profitability of ethanol conversion could be enhanced by modifying trees to yield high value coproducts, but the modifications required to produce many such materials do not seem feasible. Changes compatible with ethanol processing, e.g., solid wood products, may be workable, especially over the near-term. Stimulating production of enzymes used in ethanol processing also seems lucrative, and research on this front deserves expansion. 


\section{INTRODUCTION}

The Bioenergy Feedstock Development Program, Environmental Sciences Division, Oak Ridge National Laboratory is developing poplars (Populus species and hybrids) as sources of renewable energy, i.e., ethanol. Notable increases in adaptability, volume productivity, and pest/stress resistance have been achieved via classical selection and breeding and intensified cultural practices. Significant advances have also been made in the efficiencies of harvesting and handling systems. Given these and anticipated accomplishments, program leaders are considering shifting some attention to genetically modifying feedstock physical and chemical properties, so as to improve the efficiency with which feedstocks can be converted to ethanol.

This report provides an in-depth review and synthesis of opportunities for and feasibilities of genetically modifying feedstock qualities via classical selection and breeding, marker-aided selection and breeding, and genetic transformation. Information was collected by analysis of the literature, with emphasis on that published since 1995, and interviews with prominent scientists, breeders, and growers. Poplar research is well advanced, and literature is abundant. The report therefore primarily reflects advances in poplars, but data from other species, particularly other shortrotation hardwoods, are incorporated to fill gaps.

An executive summary and recommendations for research, development, and technology transfer are provided immediately after the table of contents. The first major section of the report describes processes most likely to be used for conversion of poplar biomass to ethanol, the various physical and chemical properties of poplar feedstocks, and how such properties are expected to affect process efficiency. The need is stressed for improved understanding of the impact of change on both overall process and individual process step efficiencies. The second part documents advances in trait measurement instrumentation and methodolgy. The importance of these and future developments is emphasized, since trait measurement constitutes the largest cost associated with adding additional traits to improvement efforts, regardless of genetic approach.

In subsequent sections, recent and projected advances in classical selection and breeding, marker-aided selection, and genetic transformation are documented and used to evaluate the feasibility of individual approaches. Interviews with specialists engaged in research and development on each approach were given particular emphasis in gauging feasibilities and defining future needs and directions. Summaries of important findings and major conclusions are presented at the end of individual sections. Closing portions describe the targeted workshop, conducted in December 1999 and list interviewees and literature cited in the text. Information obtained at the workshop was used to improve accuracy, refine conclusions, and recommend priorities for future research, development, and technology transfer. 


\section{FEEDSTOCK QUALITY AND ETHANOL CONVERSION PROCESSES}

Conversion of biomass feedstocks to ethanol generally involves using acids and/or enzymes to separate constituents and split cellulose and hemicellulose into individual sugar molecules. Simultaneously or subsequently, sugars are fermented to ethanol. Sugars derived from hemicellulose, however, are sometimes used or sold for manufacture of coproducts in an effort to raise value of the biomass and improve economics (see Coproducts). Residual lignin and other wastes are captured and partially dried for use as fuel to generate process heat, steam, and electricity. Some lignin may be separated and sold as a coproduct. Over the last several decades, a variety of conversion processes have been investigated. Although several are being used or are nearing commercialization, no clear winners have emerged and research, especially on cost reduction, continues.

Given the many origins, forms, and compositions of commercially available biomass, no single process is likely to be ideal for all feedstocks. Feedstocks containing high levels of contaminants, e.g., heavy metals, seem more amenable to acid than enzymatic hydrolysis. Moreover, processes may well have differing sensitivities to changes in feedstock quality. For these reasons, brief descriptions are given below of those processes now receiving significant research and development. Major process requirements and limitations are discussed along with general characteristics so as to highlight potential advantages/disadvantages of changes in feedstock quality.

Processes deemed most likely to be used in freeing sugars from biomass feedstocks for ethanol production include: Dilute Acid Hydrolysis, Concentrated Acid Hydrolysis, and Enzymatic Hydrolysis.

\section{DILUTE ACID HYDROLYSIS}

Regardless of process, biomass
feedstocks must be broken or shredded to
particles small enough to permit diffusion of
heat, acid, and/or enzymes into their
structures. For dilute and concentrated acid
hydrolysis, fractionation to particle sizes on
the order of chips used in commercial
pulping for papermaking is satisfactory.
In dilute acid hydrolysis, dilute acid,
e.g., $1.5 \%$ sulfuric acid, is added and the
mixture heated to moderate temperatures
(140-160 ${ }^{\circ} \mathrm{C}\left(280-320^{\circ} \mathrm{F}\right)$ ), thereby
xreleasing most hemicellulosic (C5 and C6)
sugars. Cellulosic sugar (glucose, C6) is
then isolated by raising reactor temperatures to $200-240^{\circ} \mathrm{C}\left(390-460^{\circ} \mathrm{F}\right)$. The elevated temperature readily frees glucose, but degrades some freed sugars. Substantial reductions in overall sugar yields can occur, and large proportions of C5 sugars are degraded and rendered unavailable for fermentation to ethanol or use as coproducts. In addition, degradation products inhibit organisms/enzymes used for fermentation (Gregg and Saddler 1996).

Dilute acid hydrolysis has been used on a production scale in some controlled economies, e.g., the former Soviet Union, where low yields and loss of C5 sugars are tolerated (Glassner 09/01). The process is not likely to be commercially viable in the United States without modification to a twostep system in which hemicellulosic sugars are removed prior to temperature increase. Division into separate steps, however, raises capital and operating costs. Current low prices for potential coproducts further lowers economic attractiveness. Recent research suggests that a more desirable alternative involves eliminating the high temperature step, and coupling the first low temperature step with enzymatic hydrolysis. 


\section{CONCENTRATED ACID HYDROLYSIS}

Following reduction to optimal particle size, concentrated acid, roughly twice as concentrated as that used in the dilute acid approach, and moderate temperatures, similar to those described above, are used to release both C5 and C6 sugars. Breakdown and removal are accomplished in a single step, degradation is minimal, and high yields are obtained (Goldstein and Easter 1992). Moreover, the process is robust enough to handle difficult feedstocks, i.e., those having variable composition and containing large amounts of contaminants, e.g., municipal solid wastes. Probably the most serious drawback of concentrated acid hydrolysis is the cost of purchasing and recovering acid. Some scientists (Ruth 08/18) affirm that acid recovery has been improved, although further cost reductions would help. Research on concentrated acid hydrolysis is aggressive, and the process is considered one of the most promising (Goldstein and Easter 1992).

\section{ENZYMATIC HYDROLYSIS}

Enzymatic hydrolysis, or saccharification, represents an attractive approach to breaking cellulose and hemicellulose into sugars. Since enzymes are selective catalysts and operate under mild $\mathrm{pH}$ and temperature conditions, they can be expected to give high yields and cause negligible degradation. Also, they are of natural origin and biodegradable. The challenge lies in minimizing costs of enzyme production, use, and recovery.

In contrast to acid hydrolysis, enzymatic processing requires pretreatment beyond simple reduction to appropriate particle sizes. Enzymes are large molecules, and the physical structure of biomass naturally limits access and activity. A variety of pretreatments, physical and chemical, has been evaluated. Recent research, however, seems centered on steam explosion and/or mild acid hydrolysis The latter approach is quite similar to the dilute acid hydrolysis described above. Adding dilute acid and heating to moderate temperatures frees hemicellulosic sugars, increases porosity of the residual structure, and renders cellulose susceptible to enzyme attack. Higher acid concentrations and temperatures would lessen overall yields and create breakdown products inhibitory to enzymes (Gregg and Saddler 1995). Due to some natural acidity and type of lignin, simple steam explosion may be adequate for pretreatment of poplars (Beatson 08/02).

Following pretreatment, cellulase enzymes are added to hydrolyze cellulose to glucose for simultaneous or subsequent fermentation.

Interest in enzymatic hydrolysis is great, and much research has been and is being done to resolve limiting factors and reduce costs (Wooley et al. 1999). Major research areas include: improvement of the fungi/bacteria used to produce enzymes; proportions of cellulase types; simultaneous versus sequential fermentation; reduction of hydrolysis time; batch versus continuous enzyme production and hydrolysis; and enzyme recovery (Gregg et al. 1998). Efficiency of enzyme recovery seems critical; adsorption and binding of enzymes to residual lignin removes significant quantities of enzymes from hydrolytic reactions and restricts recovery (Gregg and Saddler 1996, Sewalt et al. 1997a). Lignin quantity, rather than composition, seems the major factor (Sewalt et al. 1997b). For these reasons, modification of lignin content is more important to enzymatic processes than to acid-based processes. Nonetheless, a variety of organizations view the process with optimism and see numerous opportunities for cost reductions (Glassner 09/01). 


\section{FERMENTATION OF C5 SUGARS TO ETHANOL}

In earlier times, fermentation of $\mathrm{C} 5$ sugars was considered problematic (Gregg and Saddler 1995) due to limited effectiveness of organisms/enzymes for converting them to molecules suitable for fermentation to ethanol. Degradation products from harsh pretreatments were also found to inhibit activity. Much research was therefore devoted to improve efficiency via selection and/or genetic engineering, and suitable organisms/enzymes should soon be commercially available. Inhibitory breakdown products have been reduced via coupling with steam explosion/dilute acid hydrolysis pretreatment, but further research is needed to limit formation of such compounds, especially acetic acid. All things considered, however, individuals prominent in the field (e.g., Glassner 09/01, Jeffries 10/14) seem satisfied that C5 sugars can be used to increase overall ethanol yields and no longer view this step as an economic barrier.

Given the problems of converting C5 sugars to ethanol noted above, considerable research has also been done on using them to manufacture high-value coproducts (see Coproducts). In general, however, lucrative markets are limited. This could change in future, and markets for coproducts whose values exceed that of ethanol may expand. Cargill Corp. and Dow Chemical Inc., e.g., are forming a joint venture to manufacture biodegradable plastics from lactic acid, which is derived from xylose, a major constituent of hemicellulose. In addition, DuPont Co. recently announced a commitment to secure $25 \%$ of its revenues from nondepletable resources, rather than petroleum, by 2010. Whatever the case, conversion processes releasing $\mathrm{C} 5$ sugars in high yields with minimum degradation are likely to be preferred over others.

\section{KNOWN IMPACTS OF CHANGES IN FEEDSTOCK QUALITY}

A number of authors have published economic and sensitivity analyses of factors expected to influence conversion efficiency. To date, most such analyses have been based on simulation models and largely have been confined to evaluating impacts of volume growth, i.e., feedstock quantity and costs of harvesting, handling, transporting, and storing feedstocks. In a recent example, Grado and Chandra (1998) demonstrated that factors most affecting costs of ethanol production from woody feedstocks were process yields and harvesting costs. Plantation yield played a lesser role, and inventory size and storage time as well as conversion facility size had even lesser effects. Few published models directly address effects, positive or negative, of changes in feedstock quality. Some have addressed the issue indirectly; e.g., Gregg et al. (1998) showed that enzyme costs, reaction time, and efficiency of enzyme recovery were major limiting factors in enzymatic hydrolysis. Thus, lower lignin content and lignin more easily separated from other constituents are likely to decrease processing costs, since lower lignin content means less inactivation of enzymes as well as higher cellulose content. Wooley et al. (1999) modeled a number of factors, affirmed critical importance of feedstock growth rates and costs, and evaluated effects of elevated carbohydrate contents. A hypothetical $20 \%$ increase in cellulose and hemicellulose contents was projected to increase ethanol yields and lower processing costs. Impact of the accompanying roughly $50 \%$ reduction in lignin content, however, was said to reduce combustible residues to the extent that some biomass would have to be used to produce process heat and steam.

No recent literature was found documenting actual experimental trials of feedstocks with differing qualities. The findings noted above nevertheless suggest, 
if only indirectly, that any reduction in lignin and/or increase in cellulose contents would raise process efficiency and lower production costs. Reducing lignin content of feedstocks slated for enzymatic hydrolysis would seem to have particularly high potential for payback. Conversion efficiency would be affected favorably at several points in the process. Moreover, modest reductions in lignin content, somewhat less than the $50 \%$ reduction described above (Wooley et al. 1999), should not harm process heat, steam, and power generation, since excess electrical power is often generated and sold at prices that do not enhance overall profitability.

Regrettably, actual experimental trials may not be possible for some time. Process engineers desire relatively large quantities of feedstocks vary significantly in quality for testing. Similarly, feedstock breeders and growers would prefer to know which traits to modify before investing time and money to produce biomass in the desired quantities. This "Catch 22" situation must be voided for progress to occur. After all, changes in quality can affect processing at a variety of points and in a multitude of ways (Fig. 1). For example, denser feedstocks containing less lignin and more cellulose are likely to decrease harvesting, transportation, handling, storage, and labor costs. They could also lower both capital and operating costs by requiring smaller reaction vessels, less process steam and chemicals and/or smaller quantities of enzymes. At the far end of processing, modified lignin could also affect quantity and quality of waste water streams; chromophores released from lignin are likely to vary with lignin quantity and composition. Clearly, definitive, reallife tests are needed to determine effects of feedstock quality changes on each of the many steps in individual processes.

\section{CHARACTERISTICS OF COMMERCIALLY AVAILABLE FEEDSTOCKS}

Botanical origin as well as morphological and anatomical characteristics of biomass sources differ considerably. Noteworthy variation also occurs in chemical composition (Table 1). Most commercially available biomass materials contain roughly $35-50 \%$ cellulose. Hemicellulose, a shorter chain polysaccharide, is the second most prevalent carbohydrate. This fraction consists predominantly of C5 sugars (e.g., xylose and arabinose), with xylose being the major C5 sugar in dicotyledonous woody plants, herbaceous energy crops, and agricultural residues. Various C6 sugars and acids (e.g., glucose, mannose, galactose, rhamnose, and glucuronic acid), however, are also represented. Such sugars often contain substituent groups that contribute to formation of inhibitory acids and phenolic glucosides during processing. Lignin, a phenylpropene 3-dimensional polymer, comprises another $10-25 \%$ of the mass and is the cementing material that holds cell walls and individual cells together. Lignin cannot be converted directly to ethanol and is, therefore, either used as fuel or sold as a coproduct (see Coproducts). Lignin has an energy yield close to that of coal. The remainder consists of extractives, protein, starch, soluble sugars, and/or ash.

Based on average usable carbohydrate content, the feedstock that is the concern of this report, i.e., short-rotation poplars, clearly is well suited for conversion to ethanol. That is, poplars inherently contain 

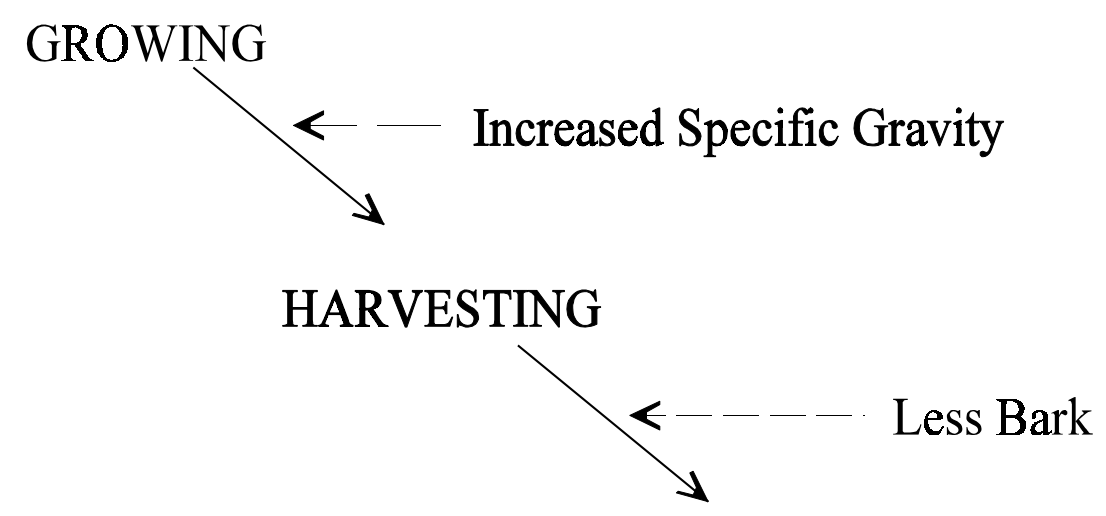

\section{TRANSPORT}

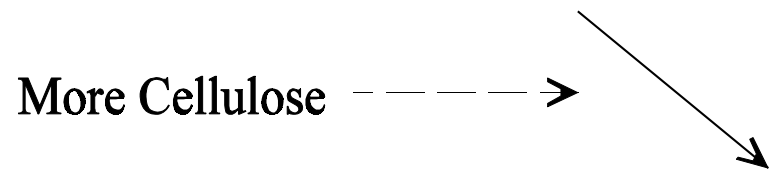

\section{PRETREATMENT}

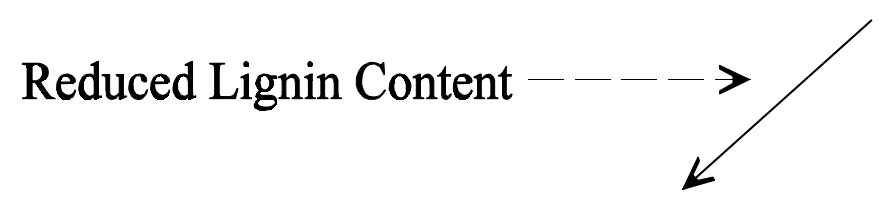

\section{CONVERSION}
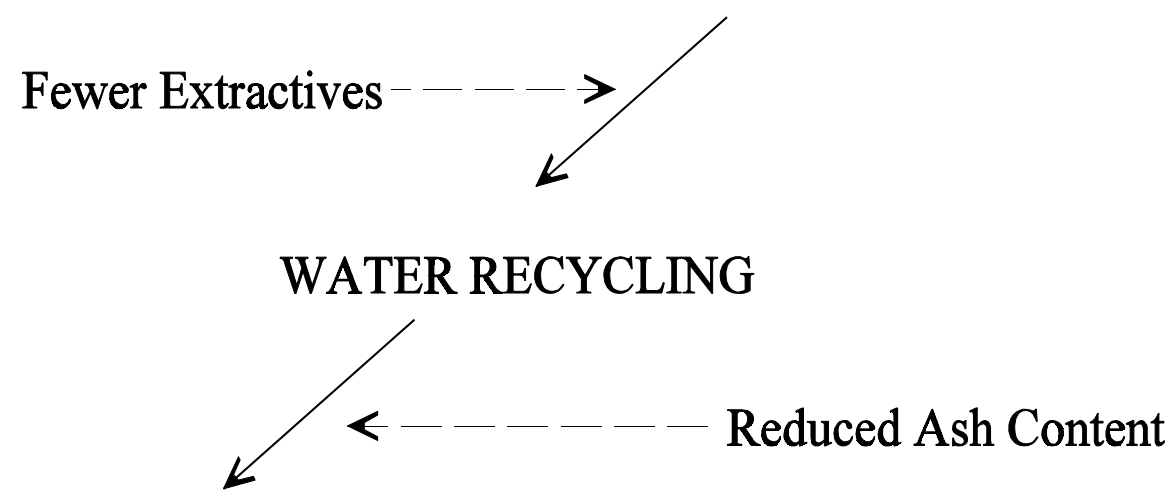

\section{WASTE DISPOSAL}

Fig. 1. Hypothetical illustration of an ethanol conversion process from tree growth to waste disposal and how genetic modification of certain feedstock quality traits might improve efficiencies of certain steps. Improvement of some qualities, e.g., lignin and cellulose contents and specific gravity, could have favorable impacts on several steps. 
Table 1. Generalized average chemical composition of major biomass sources as adapted from Wyman (1993)

\begin{tabular}{lcccc}
\hline \multirow{2}{*}{ Biomass source } & Cellulose & Hemicellulose & Lignin & Other $^{a}$ \\
\cline { 2 - 3 } & & & & \\
\hline & 50 & 23 & 22 & 5 \\
Short-rotation poplars & 45 & 30 & 15 & 10 \\
Herbaceous energy crops & 38 & 32 & 10 & 36 \\
Agricultural residues & 45 & $9^{b}$ & & 13 \\
Sorted municipal solid waste & & &
\end{tabular}

${ }^{a}$ Other $=$ extractives, protein, starch, soluble sugars, and/or ash.

${ }^{b}$ Such feedstocks often contain a further $9 \%$ of carbohydrates other than cellulose and hemicellulose.

more cellulose and less hemicellulose and extraneous materials than most other sources (Table 1), a distinct advantage if ethanol production is the objective. Viewed another way, i.e., in terms of holocellulose or the sum of cellulose and hemicellulose contents, McLaughlin et al. (1996) found that holocellulose content of switchgrass, an herbaceous energy crop, ranged from $54-57 \%$, compared to a range for poplars of 49-66\%. Recent analyses of 4- to 5-yearold hybrid poplar gave the following compositional data: cellulose $=46 \%$, hemicellulose $=24 \%$, holocellulose $=70 \%$, and lignin $=23 \%$ (Dinus 1999). Thus, short-rotation poplars, even absent genetic modification, seem as good as or better than other feedstocks considered useful for ethanol production. Although poplar lignin content tends to be higher than that of other sources (Table 1), levels are not excessive, and reduction by breeding or other means to levels in other commercially available biomass could cause dramatic increases in desirability.

\section{DESIRABLE CHANGES IN FEEDSTOCK QUALITY}

As described above, processes likely to be used for conversion of biomass to ethanol involve acid hydrolysis, enzymatic hydrolysis, or some combination thereof. Coupling dilute acid and enzymatic hydrolysis seems especially promising. No universally preferred process, however, has emerged, and a balance may be required to accommodate the many types of commercially available feedstocks. Even so, many feedstock quality characteristics are of common interest, and most will not differ markedly in effects on process types. Described below are physical and chemical properties of greatest value to most processes; exceptions are noted where appropriate.

Opportunities to match wood physical and chemical properties to ethanol conversion process requirements are manifold and have been long recognized (Dinus et al. 1990). Attempts to capitalize 
on such opportunities, however, have been limited by several concerns, not the least of which has been uncertainties about which properties to modify. Such uncertainties will persist until more information is made available concerning which traits are important, how important they are relative to one another, how their separate and combined effects influence processing, and whether increased efficiency can be effected more economically by biological means than by adjusting process technology.

Certain wood and supply characteristics are valuable regardless of process or product. For example, feedstock costs are critical since they are a major component of overall manufacturing expense. Assured supply across seasons and years is likewise important. Uniformity is also a key feature; uniform raw material is easier and cheaper to handle and process. Toward meeting these needs, past research on breeding and growing poplars for biomass energy focused on increasing adaptability, productivity, and pest/stress resistance (Tuskan 1998). Notable increases in yield and reductions in growing costs have been achieved via classical selection and breeding and intensified cultural practices (Wright and Tuskan 1998). Research on these fronts continues, and further gains are anticipated.

Viewed against this background, concerted efforts to modify feedstock quality seems timely. As noted in preceding sections, substantial ethanol yield enhancements and process cost reductions are likely to result. Higher cellulose/lignin ratios can have direct, measurable impact on process efficiency. In addition, shortened harvest rotations resulting from past improvements in growth mean that opportunities for modifying feedstock quality can now be readily exploited. Poplars routinely are propagated vegetatively, thereby allowing for rapid, inexpensive multiplication and planting of useful variants. Poplars therefore seem an ideal venue for testing and applying techniques for modifying quality.

\section{Lignin}

Reduced lignin content should be a major objective. Simply stated, less lignin equates to a higher proportion of fermentable carbohydrates and therefore higher ethanol yields. Reduction would also improve access to carbohydrates by reagents/enzymes, facilitate enzyme recovery, and lower residue and effluent quantities (Fig. 1). In addition, decreasing carbon and energy flow to lignin biosynthesis may increase amounts available for growth - a distinct advantage in terms of biomass supply and cost. On the other hand, modifying lignin biosynthesis could interfere with production of compounds needed for defense against insects and disease. The many unknowns on this front, however, should not forestall research on lignin modification. Rather, they underscore the need for adequate testing before widespread commercialization. Radical reduction of lignin content could lessen prospects for sale of high-value coproducts or necessitate purchase of process heat, steam, and power. The former prospect seems unlikely for the foreseeable future as current markets for lignin byproducts are only a million tons per year (Northey 10/06, Rasmussen 10/08). With regard to use as fuel, lignin residues often exceed requirements, and surplus electricity is sold at prices that do not add to overall profitability (Glassner 09/01). Since many currently accepted feedstocks contain less lignin than poplars (Table 1), reducing poplar lignin content to similar or lower levels is not likely to increase fuel and power costs.

Modifications of lignin composition and structure may also be helpful, although there is much uncertainty concerning what to modify and how any changes would affect processing. Desirable changes would 
be those that ease access to and removal of carbohydrates by heat and acids or enzymes, improve recycling of acids and enzymes, facilitate removal of lignin residues, and reduce volumes and chromophore contents of effluents. Lessening frequency of covalent linkages between lignin and hemicellulose would be especially advantageous. Changes might also be made to render lignin residues more useful for manufacture of coproducts having higher value than fuel.

Some investigators have long advocated increasing the proportion of syringyl to guaiacyl lignin precursors, i.e., raising syringyl/guaiacyl ratios (SGR). Syringyl monomers contain one more methoxyl group than guaicyl monomers, thereby reducing opportunities for crosslinking in native lignin. During the workshop conducted to review this report, however, participants argued against such changes since high SGR clearly facilitate alkaline pulping as used in paper manufacture, but would not ease carbohydrate removal in the acidic environments common to ethanol conversion processes (Chiang 12/07, Sewalt $12 / 07$, and 03/02). Indeed, lignin that is less condensed in vivo could be more of a problem than benefit. Degradation could occur, with breakdown products becoming mixed in the process stream to interfere with carbohydrate breakdown, fermentation, and separation of residues. Effluent volumes could also rise and perhaps contain more undesirable chromophores. Modifications causing lignin to be less reactive in acidic processing might be a better goal.

Until more is known about the benefits of modified lignin composition and structure, research and related efforts seem best concentrated on reducing lignin content.

\section{Cellulose}

Conversion of biomass to ethanol entails breakdown of cell wall carbohydrates to sugars and fermentation of said sugars to ethanol (Fig. 1). Accordingly, higher cellulose content directly translates to greater ethanol production and process efficiency. The inverse relationship between lignin and cellulose contents underscores advantages of reducing the former. Raising cellulose content per se, however, may bring additional benefits. For example, evidence is accumulating that cellulose content can be increased via genetic transformation, and that such modification increases growth (see Transformation). In addition, results from classical genetics research indicate positive correlation between specific gravity and cellulose content (see Classical Selection and Breeding). Higher specific gravity often results from increased proportions of fibrous cells and thicker cell walls. The thickening generally occurs in secondary cell walls where the concentration of cellulose is much greater than that of lignin. Thus, cellulose content and traits affecting it, e.g., lignin content and specific gravity, deserve consideration in efforts to modify feedstock quality.

\section{Hemicellulose}

Although means for reducing hemicellulose content are not immediately available, reduction would increase the amount of cellulose available for conversion to ethanol. Modifying hemicellulose composition may also prove beneficial. For example, recent evidence suggests that reducing lignin content via genetic transformation also provokes changes in quantities of C5 and C6 sugars (Hu et al. 1999). Increasing the proportion of $C 6$ 
sugars in hemicellulose, e.g., glucose, would raise overall process efficiency. Also, possibilities should be investigated for reducing number and length of side chains and reducing acid content so as to diminish frequency of linkages to lignin, decrease acid formation during processing, raise solubility, and improve access to process reagents. On the negative side, changing hemicellulose quantity and/or quality, as outlined above, would diminish quantities of xylose available for coproduct manufacture, and potentially decrease desirability of the biomass for paper manufacture. Growers could lose alternative markets and/or suffer from lower prices in those markets.

\section{Specific Gravity}

Increasing specific gravity along with volume productivity per acre is expected to have significant impact on conversion efficiency. Improving volume growth alone, of course, does much to increase feedstock supply and reduce costs. By also raising specific gravity, however, total mass of carbohydrate would be increased above and beyond that associated with rapid growth. Higher specific gravity primarily results from increases in fibrous cell numbers and thicker cell walls, i.e., total wood substance. Since secondary cell walls tend to have a higher cellulose to lignin ratio, total carbohydrate mass would be increased. As noted above, genetic correlations between specific gravity and cellulose content generally are positive; thus, raising specific gravity results in greater mass-more carbohydrate, less air, and potentially lower moisture content. Greater mass per unit volume means more carbohydrate harvested per unit area and per unit of harvesting equipment, transported per unit load, and stored per unit inventory capacity (Fig. 1). Lower costs should, therefore, accrue in these process steps. Greater mass of carbohydrate also means that more ethanol can be produced per unit processing equipment and time, since conversion equipment is density rather than volume dependent. Indeed, a processing unit of modest size using high specific gravity wood should be less expensive to operate than a larger one supplied with greater volumes of lighter wood. Given that genetic correlations between specific gravity and growth tend to be negative, improving both traits will require some extra effort by breeders. More selections will have to be bred and more offspring evaluated to ensure progress on both fronts.

\section{Moisture Content}

Lower moisture content would have much the same benefits as higher specific gravity. Less moisture means more wood substance manipulated during harvesting, transportation, storage, and processing (Fig. 1). As reported earlier (Dinus et al. 1990), some potential exists for reducing moisture content by means other than physical drying after harvest and/or in storage. That is, moisture content is under some degree of genetic control; heritabilities are variable but encouraging, and the trait is not strongly correlated, positively or negatively, to other quality traits. Some poplar breeders have expressed interest in the trait, but uncertainties are manifold. The trait is affected by a host of developmental, age, seasonal, and environmental variables. Since little new information has accumulated, modification must be preceded by more definitive research.

\section{Extractives}

Some compounds in this category, e.g., storage compounds such as fats, alcohols, and waxes, may contribute to ethanol yields. Starches and soluble sugars would have positive effects, but quantities are quite low in poplars. In consequence, overall effects of extractives could be negative. Some compounds, e.g., tannins, 
even if present in low concentrations, interfere with activity of hydrolytic and fermentative enzymes. In addition, certain extractives have been implicated in pest resistance. In general, roles, functions, and inheritance patterns of extractives remain unclear. Given the relatively low concentrations of extractives in poplar, roughly $1.2 \%$ on an O.D. wood basis (Dinus 1999), a better understanding of this trait and its components seems necessary before attempts are made to modify it. If modification is attempted, emphasis should placed on components known to inhibit processing and/or associated with waste disposal problems. Viewed another way, molecular genetics research could help untangle this puzzle.

\section{Ash}

Quantity/quality of ash can affect several process steps. Impact, however, should be minimal, given the relatively minor mass of ash in poplar wood, usually only a bit more than a half percent. Nonetheless, reducing residuals, including ash, could lower waste treatment and disposal costs. More importantly, certain elements, e.g., heavy metals, interfere with process chemistry, whether acid or enzymatic hydrolysis, and lower ash quantities would therefore benefit conversion efficiency, especially if enzymes, organisms, chemicals, and/or process water are recycled. In addition, ash could accumulate to harmful levels as residues are concentrated for use as boiler fuel. Ash is a major contributor to boiler scaling and slagging. Less ash equates to less scaling and slagging, leading in turn to more efficient generation of heat, steam, and electricity as well as lower boiler maintenance costs. An earlier report (Dinus et al. 1990) provided some, though conflicting, support for modifying ash content via genetic manipulation. Since ash content of bark tends to be substantially greater than that of wood, reduction might be better accomplished by modifying bark quantity. Any efforts to effect change, however, should be preceded by research on genetic variation, heritabilities, genetic correlations, and genotype $\mathrm{X}$ environment interactions. The last aspect is particularly important as both wood and bark ash contents naturally tend to be higher in regions having calcareous soils.

\section{Bark}

Harvesting, handling, and processing technologies are such that bark will not be removed before hydrolysis and fermentation. Since bark properties differ from those of wood, they can have different effects on processing. On the positive side, bark contains substantial quantities of carbohydrates. On the negative side, it also contains more extractives and ash than wood. Past reports indicate that bark thickness and proportion vary greatly with position within tree as well as tree size and age, growth rate, and site. Information on genetic parameters is scarce, and reliable estimates should be secured before modification is attempted. Given importance of lower bark/wood ratios as well as ash and extractives, research to acquire needed information seems warranted.

\section{SUMMARY AND CONCLUSIONS}

In sum, preferred feedstock characteristics include less lignin, more cellulose, and higher specific gravity. Lignin composition and structure could be important, but research is needed to identify which issues to address. Reduced moisture content seems of lesser importance.

Importance of extractives is uncertain, but benefits might accrue from lowering amounts of components likely to inhibit process reagents and/or pose waste treatment and disposal problems. Lower 
bark quantities would be advantageous, especially since they would also reduce ash and extractives contents. All such changes should be sought only in the context of accelerated growth rates and maximum uniformity.
With the relative importance of traits affecting feedstock quality having been noted above, the remainder of this report focuses on the key issues, i.e., modification of lignin and cellulose contents and specific gravity via classical selection and breeding, marker-aided selection and breeding, and/or genetic transformation. Side effects on other traits caused by such modification are documented to the extent possible.

\section{MEASUREMENT OF FEEDSTOCK QUALITY}

Until recently, most organizations engaged in genetic modification, especially classical selection and breeding programs, whether aimed at solid wood, paper, or energy products, either did not consider wood physical and chemical properties or assigned them a low priority (Zobel and Jett 1995). A variety of reasons have been advanced to explain this low level of activity, e.g., uncertainties about which traits to modify, limited genetic information, and high measurement costs.

Costs of trait measurement are a particular concern, since they constitute the largest share of expenses associated with adding a trait to breeding programs and assessing the impacts of modification, regardless of approach. Ideally, traits should be measurable via low cost, rapid methods that use small, nondestructively collected samples (whole or partial increment cores). Wood volume, a typical measure of productivity, can be measured for pennies per tree. At the other extreme, traditional wet chemistry assays of wood chemical constituents can take days or weeks and cost \$500-1000 per sample (Dinus 1999). In addition, comprehensive chemical assays via traditional methods often requires degrading one or more components to measure others, leaving some to be measured by subtraction. Moreover, traditional methods often require large samples; $10 \mathrm{~g}$ or more of O.D. wood for chemical analyses and whole disks for specific gravity determinations. Obtaining such quantities can require harvesting of entire trees, thereby eliminating valuable genotypes or necessitating expensive preservation measures. In addition, genetic modification via classical selection and breeding involves testing large numbers of parents and offspring, and measurement costs can easily exceed the budgets of even the most robust programs.

Fortuitously, interests of the biofuels industry coincide with those of the forest products industry. In recent years, wood products and paper companies acting alone, collectively, or cooperatively with university and government laboratories have intensified development of improved instruments and methods for measuring quality traits. This heightened activity is testimony that genetically modifying wood properties is not only feasible but also profitable. Indeed, development of reliable, rapid, and low-cost methods requiring small, nondestructively collected samples is well under way. Advances have been considerable and research is accelerating. Examples applicable to assessing biomass feedstock quality are discussed below. 


\section{LIGNIN, CELLULOSE, AND HEMICELLULOSE CONTENTS}

Traditional methods for assessing lignin content tend to be labor intensive and costly (Dinus and Welt 1997, Dinus 1999). Recent innovations in instrumentation and methods have increased convenience as well as lowered costs (Purnell 10/18). Some example methods include reflectance near infra-red (RNIR), Fourier transform infrared (FTIR), and pyrolysis molecular beam mass spectrometry (pyMBMS).

RNIR and FTIR assays have been evaluated and are being utilized by a variety of organizations. Wright et al. (1990) found that results from RNIR analyses of powdered wood (5 $\mathrm{g}$ or less) were correlated, significantly and positively, with those of traditional analyses for cellulose contents of and pulp yields from several pine species. Michell and Schimleck (1995) used a similar approach to predict chemical needs for and pulp yields from Eucalyptus species. The same authors suggested that their method could also be used to estimate cellulose, xylan, and lignin contents (Schimleck and Michell 1998). Using a similar approach, Clarke and Wessels (1995) measured holocellulose, alphacellulose, and lignin contents of several Eucalyptus species. Samples were small amounts of sawdust collected while preparing samples for traditional assays. After calibration to baseline wet chemistry data, the equipment could assay up to 60 samples per day. Costa E Silva et al. (1998) found FTIR analysis a reliable means for determining lignin content and its correlation to growth and wood density in 9-year-old Picea sitchensis. Crocker (1995) reviewed literature on RNIR and FTIR applications through 1995 and used FTIR to estimate genetic variation in and heritability of lignin content in Pinus taeda.

RNIR and FTIR capabilities are available at a variety of U.S. industrial, university, and government laboratories.
Costs are less than $\$ 100$ per sample for comprehensives assays and substantially lower for estimates of only major constituents.

A relatively new approach, pyMBMS, has been tested on a variety of lignocellulosic materials including wood, herbaceous fiber, and pulps; results correlate well with those from traditional wet chemistry (Tuskan et al. 1999a). pyMBMS provides estimates of virtually all constituents, i.e., contents of lignin, cellulose, hemicellulose, and most individual hemicellulosic sugars along with proportions of syringyl and guaiacyl lignin.

The method requires only $10-15 \mathrm{mg}$ of O.D. ground wood, is rapid (about 3 min per duplicate sample), and is relatively inexpensive at $\$ 15$ per sample (Tuskan 08/01). Davis et al. (1999) and Tuskan et al. (1999a) deemed pyMBMS reliable and affordable for assaying genetic variability in wood chemical properties. Undoubtedly, it could also be used to confirm changes effected by genetic transformation.

Viewed in another dimension, comprehensive pyMBMS assays might prove useful for detecting natural variation in lignin composition, thereby leading to identification of mutants such as the bmr alleles in corn. Only one such mutant has been found in trees, the null CAD allele in Pinus taeda (MacKay et al. 1997), but others are likely to exist (Sederoff 09/29). Such mutants could prove useful in modifying lignin quantity/quality, whether by selection and breeding, marker-aided selection, or genetic transformation.

Taking a different tack, Donaldson et al. (1997) measured lignin contents of middle lamellae in wood from 16-year-old Pinus radiata. High clonal repeatabilities were observed, and the trait seemed an inexpensive indicator of overall lignin content.

Lignin quality assay has also received attention in recent times as a result of accelerated research on genetic transformation. Capabilities have expanded 
and, more importantly, costs have fallen. Combined lignin content and SGR assays can now be performed on less than $1 \mathrm{~g}$ O.D. ground wood for $\$ 250$ or less per sample (Dinus 1999).

\section{SPECIFIC GRAVITY}

Methods for measuring specific gravity are discussed here because of its utility in raising dry wood substance production and potential use as an indirect means of increasing cellulose content. Densiometric techniques have been used for years, but recent improvements have increased utility and reduced costs. Sample collection has likewise been improved.

Specific gravity is a complex trait, i.e., it is a composite of several underlying traits. Consequently, increases in specific gravity can be the result of any one or more individual causes. The situation is especially complicated in hardwoods since changes in cell types, numbers, and sizes are all involved (Zobel and van Buijtenen 1989, Dinus et al. 1990).

Accordingly, assay methods have been refined to quantify not just specific gravity but also individual contributing factors, e.g., cell wall thickness. In response to demands from Australian forest products companies, Evans and Downes (1995) developed an automated system (Silviscan), combining X-ray densitometry and image analysis, to measure softwood specific gravity, cell size, and cell wall thickness. Instrumentation and software are capable of analyzing samples prepared from $12 \mathrm{~mm}$ pith to bark increment cores at the rate of 4 $\mathrm{mm} / \mathrm{min}$, or perhaps 10,000 per year. Costs for comprehensive analyses are projected at $\$ 100$ per sample. By resolving specific gravity into its various components, techniques such as Silvascan provide more useful information on wood quality than earlier methods. Efforts are under way to further automate the system and extend application to hardwoods, initially
Eucalyptus species.

Tuskan et al. (1999a) used computer tomography X-ray densitometry for comprehensive assay of specific gravity in hybrid poplar. The method utilizes X-ray scanning of dry, preferably extracted, 5 or 12 $\mathrm{mm}$ increment cores. Preparation and machining beyond extraction and drying are not necessary. Specific gravities of early and late wood, juvenile and mature wood, and/or whole cores can be obtained via postscanning software processing. The method is fast, roughly 30 cores every $18 \mathrm{~min}$, with costs averaging $\$ 10$ per inch of core, or roughly $\$ 60$ for a 6 in DBH tree (Tuskan 08/01).

As noted above, advances in sample collection methods have also accrued. A variety of all-terrain vehicles, fitted with hydraulically or electrically driven corers, have been developed for extracting increment cores. Using such a system, Crocker (1995), working with two other individuals, identified, marked, and collected $12 \mathrm{~mm}$ bark-to-bark cores from 123 35-year-old Pinus taeda trees in less than $8 \mathrm{hr}$. This may not be a record but clearly illustrates the extent to which efficiency has been improved.

Several short-cut, indirect procedures have also been developed to expedite and cheapen collecting specific gravity data. One example is the Pilodyn, a hand held instrument that drives a flat-nosed steel pin into wood samples with known force (Raymond 1995). Degree of penetration is read from a convenient scale: the less the penetration, the greater the specific gravity. As a result, Pilodyn observations vary inversely with specific gravity. Breeders of Eucalyptus species routinely use the instrument for measurements in situ. Raymond (1995) reported quite strong heritabilities as well as phenotypic and genotypic correlations. Tibbits and Hodge (1998) assessed genetic variation in and heritability of basic wood density in 4- to 8-year-old Eucalyptus nitens, and found 
strong genetic correlations between Pilodyn readings and densities determined via the traditional immersion method. Their findings confirmed those of Greaves et al. (1996) for the same species. According to Costa E Silva et al. (1998), Pilodyn measurements taken on 9-year-old Picea sitchensis were sufficiently reliable to construct selection indices for improving yield and wood quality. These authors considered Pilodyn measurements an inexpensive, fast, and reliable substitute for more costly traditional measurements.

For short-rotation poplars, on the other hand, Rousseau (09/29) indicated that the Pilodyn, as presently configured, has limited utility. Desired levels of precision were not obtained even after bark removal and multiple measurements. Considerable variation also occurred among users. He suggested, however, that the instrument could prove useful for culling individuals with particularly low specific gravity. One cannot help but speculate that this or a similar instrument can be developed for poplars. The payback from fast measurements on standing trees could be substantial.

\section{SUMMARY AND CONCLUSIONS}

The foregoing examples illustrate that trait measurement methods and their costs should no longer be considered obstacles to modifying feedstock quality. Measurement techniques that are reliable, rapid, and inexpensive, and that use small, nondestructively collected samples have become available. Rising interest around the world in improving wood properties ensures further advances in instrumentation and methodology. Expanded research and development on modification of feedstock quality should likewise bring additional capabilities and wider availability. Further cost reductions can also be expected as user demand increases and independent/cooperative testing laboratories enter the field.

Several cautionary notes, however, must be mentioned (Purnell 10/18). Small, nondestructively collected samples are essential, but provision must be made to ensure that results are representative of whole-tree characteristics. In addition, methods and analyses must be tailored to need. Tools should fit tasks, and the simpler and more useful the outcome, the better. General interpretation of RNIR analyses, e.g., lignin content, may be sufficient for practical breeders. In contrast, more thorough analyses, e.g., pyMBMS assay of lignin composition, may be needed for genetic transformation. The goal should be just the right data, detail, and reliability at lowest cost. 


\section{CLASSICAL SELECTION AND BREEDING}

Effective, efficient selection and breeding requires that traits of interest possess usable levels of genetic variation, meaningful degrees of genetic control, and favorable correlations with other important traits. Stability across ages and environments is also important. Since trait measurement constitutes a major share of breeding activity and cost, assay methods must be inexpensive, reliable, and convenient.

In an earlier review, Dinus et al. (1990) summarized available information on traits affecting feedstock quality and provided tables of means, ranges, heritabilities, and correlations among traits. More recently, Zobel and Jett (1995) reviewed world literature on genetic control and modification of wood properties. Like its predecessor publication, Zobel and van Buijtenen (1989), this book concentrated largely on physical properties. Both publications, however, contain valuable data on variation in, heritablility of, and relationships among important chemical traits. A wealth of information on Eucalyptus species is provided in the proceedings of an outstanding Australian symposium (Potts et al. 1995). More recently, Dinus and Welt (1997) adapted information from these sources and added new data in an effort to demonstrate feasibility of improving wood and fiber properties via genetic modification. Both chemical and physical properties were emphasized, as were advances in measurement methods.

In this section, the aforementioned reviews are used as a baseline, and are updated with findings from more recent literature and personal communications with poplar breeders and growers. Information on genetic variation in and control of lignin and cellulose contents in short-rotation poplars is emphasized, with an eye toward determining the feasibility of modifying them. Specific gravity, given its more or less direct relationship to cellulose content, is also discussed. Hemicellulose content is given nominal attention.

\section{LIGNIN}

Reviews cited above indicated existence of genetic variation in lignin content among and within species. Though statistically significant in some reports, however, variability among genetic entities was small, often only a few percentage points but sometimes as large as nine percentage points. Heritabilities were low to moderate. Little data were found on relationships between lignin content and other traits, but most authors considered it independent of growth. Many older reports, however, raised more questions than answers in that experiments involved small numbers of families or clones, limited replication and only one or a few test sites.

On the whole, results in current literature are from larger, more robust experiments. As one example, Bertolucci et al. (1995) reported findings from a replicated, six-year-old, multi-site trial of Eucalyptus grandis hybrids. Results seem especially relevant since the test involved only nine elite clones previously selected for growth rate. Variation among clones in lignin content was significant, and broad sense heritability on a clone mean basis was estimated as 0.82 . Clone $\mathrm{X}$ site interaction was also significant, indicating the need to identify clones stable across sites or deploy individual clones to sites on which they perform to maximum potential.

Correlations between lignin content and other traits were not reported.

Variation in lignin content, assayed via FTIR, was measured for 30 clones of nine-year-old, fast-grown Picea sitchensis 
(Costa E Silva et al. 1998). Variability was limited, but heritability was high, suggesting that reduced lignin content could be obtained, albeit slowly, via selection, propagation, and planting of the best clones. Some difficulty was expected, however, in that lignin content varied directly with growth. Selecting for reduced lignin content could therefore limit genetic gain in growth rate. Adverse effects on growth might be avoided if and when genetic parameters and appropriate economic weights are combined in a selection index. In ongoing hybrid poplar research sponsored by the Bioenergy Feedstock Development Program, wood physical and chemical properties were quantified via traditional analyses (Dinus 1999). Assayed were one Populus trichocarpa and two Populus deltoides parents, plus four F1 offspring and ten F2 individuals. Lignin content averaged $23.4 \%$, and varied from $20.5-25.4 \%$, a range of 4.9 percentage points. SGR values averaged 2.4 , and ranged from 2.0-2.6, a differential of 0.6. Means and ranges from a related test of the same parents and F1 hybrids were similar, even though sample trees had been grown in widely different environments. Phenotypic variation in these trials were somewhat larger than that in some older trials described above, possibly a result of sample trees having been different species and interspecific hybrids. In related experiments, 16 interspecific hybrids, derived from several species, showed even larger ranges in both lignin content (9.3 percentage points) and SGR (1.1). In all three of the aforementioned trials, trees with low lignin content tended to have high SGR.

Such findings suggest that the limited variability in lignin content observed within species can be increased via interspecific hybridization. This should not be difficult or costly since most U.S. poplar breeders already utilize interspecific hybridization to some extent. Moreover, the approach seems attractive in view of the variety of interfertile species available for creation of hybrids and backcrosses (Stettler et al. 1996).

Evidence has also accumulated that breeding to increase growth and yield favorably alters lignin properties. Wood from improved Pinus radiata showed different fracture patterns than that from nonimproved controls (Donaldson 1995). Differences were attributed to lesser lignification in the boundary between the $\mathrm{S} 1$ and S2 layers in cell walls of improved trees. Frequency of phenolic groups in lignin may also have been a factor.

Concerning correlations between lignin content and other traits, the aforementioned reviews provided little in the way of reliable data, and little new information was found in recent literature. Malan and Arbuthnot (1995) summarized available information on wood properties of Eucalyptus grandis grown in South Africa. Phenotypic correlations between lignin content and specific gravity were modest and mostly negative. Costa E Silva et al. (1998) found an unfavorable relationship between lignin content and growth of young Picea sitchensis. More and better information on trait relationships is badly needed.

So-called brown midrib or bmr mutations that reduce lignin content or modify its properties have been identified in corn (Zea mays). Another affecting lignin biosynthesis is known in Arabidopsis thaliana. In corn, one or more bmr mutants is being incorporated into otherwise improved inbred lines via backcrossing. These are then outcrossed to create low lignin hybrid varieties (Keeling 09/03, Hettenhaus 09/01) for dairy silage with improved digestibility and yield. Such varieties may also favorably affect ethanol production from corn stover, and the National Renewable Energy Laboratory plans to evaluate several in the near future (Thomas 10/08). Just recently, a mutation 
affecting lignin biosynthesis was found in loblolly pine (MacKay et al. 1997). Recent advances in chemical and genomic analyses should facilitate discovery of additional mutations. Poplars are logical candidates to search, and the ease with which they can be bred and cloned make them an ideal venue for deploying mutant alleles affecting feedstock quality.

Over a decade ago, Savidge (1986) analyzed prospects for genetically improving wood properties and argued that genetic control of lignin content was independent of that governing cellulose content and specific gravity. He concluded that lignin content could be reduced without harming tree growth and development. In general, these arguments still hold, although much information is still needed.

Modifying lignin content via classical selection and breeding is possible, but opinions differ on ease and cost of effecting change. Heritabilities, though variable, are as strong as or stronger than those for growth traits, but the narrow range of variability will limit progress. Nevertheless, most breeders consulted during course of this review were optimistic, believing that even small changes could have dramatic financial impact.

\section{CELLULOSE}

Despite its importance, relatively little information is available on genetic variation in and control of cellulose content beyond that provided in the reviews noted above. Most recent information springs from research on Eucalyptus species, and findings are not always consistent. In general, cellulose content varies roughly from 7-15 percentage points among genetic entries examined in most published trials, new or old, a range exceeding those for lignin and hemicellulose contents.

Examples from earlier literature illustrate the problems of and/or opportunities in attempting to alter cellulose content. Substantial genetic variation in cellulose content was found among trees within loblolly pine families (Jett et al. 1977). Much of the variation, however, was nonadditive, thereby making the classical pine breeding approach impractical.

Substantial gains, however, seemed possible via cross pollination of outstanding trees and clonal propagation and planting of the best progeny. Some years later, this approach was employed with Eucalyptus hybrids by Aracruz Florestal S.A. in Brazil (Zobel et al. 1983). Breeding and selecting, in turn, for growth, specific gravity, and cellulose content coupled with clonal propagation increased pulp yields from 47 to $51 \%$. Parallel increases in uniformity were also obtained and most likely contributed to reduced harvesting, transport, and processing costs.

Olson et al. (1985) found only minor genetic variation in and control of holocellulose in 75 clones of Populus deltoides three years after planting at one location. Variation in alpha-cellulose content, however, was larger, varying from 48.2 to $55.8 \%$. Broad sense heritability (0.34) was stronger than that for both holocellulose content and growth. The authors considered both variability in and heritability of alpha-cellulose content sufficient for genetic improvement.

Correlations with growth, however, were negative, and simultaneous improvement seemed impractical, i.e., selecting for cellulose content was seen as reducing gains in growth. The authors nonetheless recommended that materials selected and preserved for advanced generation breeding include sufficient clones to maintain variability in alpha-cellulose content. This recommendation recognized that correlations are imperfect and subject to change with age, and that trait valuations may likewise change with time. Imperfect correlations mean that screening more individuals could yield selections excelling in both growth and cellulose content. The 
issue becomes one of how much breeders can afford to spend on the additional effort.

A survey of published estimates for genetic parameters of wood and fiber properties in Eucalyptus species (Raymond 1995) found that genetic control of most traits was moderate to strong. Individual tree and family heritabilities for kraft pulp yield, an indirect measure of cellulose content, ranged from $0.30-0.56$ and $0.18-0.62$, respectively. Phenotypic and genetic correlations between pulp yield and basic wood density varied from $-0.24-0.31$ and $-0.11-0.67$, respectively. Those between pulp yield and growth, though negative, were weaker.

In contrast, Bertolucci et al. (1995)

did not find significant variability in screened pulp yield among selected Eucalyptus clones, and broad sense heritability was lower than those for most other traits. The authors, however, did find that wood consumption varied significantly, and was under strong genetic control. Wood consumption was defined as the quantity of wood required to produce a ton of pulp; the lower the consumption, the greater the efficiency and profit. The differential was said to result from the impact of lignin and extractives content on pulp yield, whereas wood density was the major factor influencing wood consumption. Wood density varied significantly and proved highly heritable.

Clarke and Wessel (1995) evaluated wood properties of trees from a six-year-old Eucalyptus species/provenance trial in South Africa. Nine species, each represented by several provenances, were represented. Cellulose, pentosan, and lignin content varied significantly among species; only cellulose and pentosan contents, however, varied among provenances within species. Cellulose content ranged from $48-55 \%$, pentosans from $17-22 \%$, and lignin from $20-24 \%$, in line with those given elsewhere herein.
In the hybrid poplar investigation described above (Dinus 1999), cellulose contents averaged $46 \%$, ranging from 42.0-52.6\%. This 10.6 percentage point range is in line with or larger than earlier estimates for poplar.

In sum, genetic variability in cellulose tends to be larger than that in other chemical constituents. Interspecific hybridization seems an effective way to further increase variation. Although quite variable, broad sense heritabilities, on the whole, seem sufficiently large for long-term selection and breeding, provided that outstanding individuals are clonally propagated and planted. If naught else, the rather strong relationships between cellulose content and specific gravity and/or cell wall thickness provide an indirect means for improvement. In consequence, lignin contents would be reduced proportionately. Several inconsistencies nevertheless persist. Some reports show cellulose content and specific gravity varying inversely with growth, while others indicate the opposite. Even though such correlations are weak, they imply that breeding for cellulose content, directly or indirectly, could have adverse effects on gains in growth rate. The issue becomes one of how much breeders can afford to spend on selecting, breeding, and propagating outliers that excel in both traits.

Breeders would be helped by more and better data on variation in and heritability of cellulose content, and especially on genetic relationships among traits. The many energy and industrial plantations established over the past two or so decades contain a wealth of material for such research and should be exploited to the fullest possible extent.

\section{HEMICELLULOSE}

Earlier reviews, e.g., Dinus et al. (1990), disclosed only nominal variation in poplar species and hybrids. Though statistically significant in some instances, 
the limited variability was considered inadequate for breeding. In contrast, recent analysis of nine Eucalyptus clones showed significant variation and rather strong genetic control (Bertolucci et al. 1995). Assays of representatives from two poplar species and their hybrids (Dinus 1999) showed phenotypic variation in hemicellulose content on a par with that for lignin content. Further research on genetic variation and control as well as implications for energy production may be warranted, particularly in view of the potential for increasing C6 sugar content and lessening frequencies of covalent linkages to lignin.

\section{SPECIFIC GRAVITY}

Specific gravity or basic wood density is important in manufacture of numerous products. New, less expensive, and more comprehensive measurement methods have fostered use of the trait as an index of wood quality. Positive correlations with dry wood substance and cellulose content suggest that improvement could enhance ethanol production. Even so, determining specific gravity still costs more than measuring growth (Williams and Neale 1992). Thus, decisions to include the trait require clear understanding of costs and benefits.

Though often viewed and manipulated as a single trait, specific gravity is a composite trait dependent primarily upon proportions of late to early wood, cell type, size and number, and cell wall thickness. Chemical composition, e.g., relative contents of lignin and cellulose, also affect the trait but to uncertain degrees. In softwoods, e.g., Pinus taeda, proportions of late to early wood and thickness of latewood fiber walls have greatest influence. Specific gravity of hardwoods is governed by these factors as well as by proportions and sizes of a wider variety of other cell types.

Factors contributing to specific gravity can vary together or independently, and individual trees can have similar specific gravities for different reasons. Given the potential for variable causes and outcomes, specific gravity can be altered by changing one or more contributing factors.

Benefiting from efforts to increase specific gravity therefore requires understanding what is changed and how that in turn affects process and product.

As recounted in reviews cited above, genetic variation in specific gravity is significant and more than adequate for improvement. Heritability values range from roughly 0.4 to 0.7 , values as high or higher than those for any other traits of interest to commercial tree breeders. Thus, opportunities for modifying specific gravity by classical selection and breeding are excellent.

In recent experiments involving several poplar species and hybrids, Herpka (1990) found that specific gravity and volume growth varied significantly among families and clones within families. Broad sense heritabilities for specific gravity were moderate to high, and greater than those for growth. The relationship of specific gravity to both volume growth and dry wood production per unit area, though negative, generally was weak. According to the author, variability in and heritabilities of survival, growth, and specific gravity were so large that trees outstanding in terms of dry wood production could be selected without difficulty, even though the traits were negatively correlated.

In a survey of literature on Eucalyptus species grown in Brazil, Ferreira and Santos (1995) described results from young plantations of six species and a variety of provenances. They concluded that wood density varied substantially, especially among species. Correlations between density and wall thickness were considered useful.

Raymond (1995) surveyed literature on breeding of Eucalyptus species and documented heritabilites for various wood properties. In general, basic wood density, 
whether measured directly or estimated via Pilodyn readings, was under rather strong genetic control. Family and individual tree heritabilities for density per se, though variable, averaged 0.65 and 0.57 , respectively. Individual tree heritabilities for Pilodyn penetration readings averaged 0.49 . Genetic correlations between traditional density determinations and Pilodyn measurements were, as expected, negative and strong. Relationships between density and tree height and volume or growth were highly variable.

Basic wood density varied significantly among nine elite Eucalyptus clones (Bertolucci et al. 1995). Broad sense heritability was the highest found for any wood property, and density was considered the primary factor contributing to variation among clones in terms of wood consumption per ton of pulp. Wood density was therefore deemed an important trait for future breeding.

Results from a well-replicated, multisite trial of Eucalyptus nitens showed significant genetic variability in wood density (Tibbits and Hodge 1998). Trees from six provenances and 300 openpollinated families were measured at seven years of age. Narrow sense heritabilities for density and pulp yield, a proxy for cellulose content, were 0.42 and 0.37 , respectively. Both exceeded that for basal area growth (0.19). Neither density nor pulp yield exhibited significant genotype $\mathrm{X}$ environment interactions. Limited Pilodyn measurements of density gave a heritability of 0.46 , and were strongly correlated with density assayed via the traditional immersion method. Genetic correlations of pulp yield to density and growth were favorable though modest. That between density and growth, however, was unfavorable but weak.

In conjunction with evaluations of lignin content of young Picea sitchensis, Costa E Silva et al. (1998) also examined variation in and inheritance of Pilodyn estimates of specific gravity. Variation was substantial and inheritance strong. As in the case of lignin content, however, the correlation with growth was unfavorable.

Malan and Arbuthnot (1995)

summarized literature concerning variation in and relationships among wood density and related traits of Eucalyptus grandis grown in South African. Density was not influenced greatly by ray or vessel characteristics, but was related to fiber wall thickness and fractional volume of cell wall substance. Phenotypic correlations between density and lignin content were generally negative and strong. In contrast to other reports, however, those involving cellulose were not significant.

As discussed above, phenotypic and genetic correlations between specific gravity and other traits vary greatly. Those with latewood proportions and fiber wall thickness are positive and strong. On the whole, those with cellulose also tend to be positive and useful. Associations with growth, however, range from slightly negative to slightly positive, but typically are weak. Given this weakness, breeders have ample opportunity to find trees for breeding and cloning that excel in both traits and that are likely to yield large quantities of dry wood substance. In the final analysis, this becomes a numbers game and is decided by how much additional effort can be devoted to selecting and testing the extra trees needed to break the correlation and identify productive outliers.

One correlation, often not given sufficient attention, is that between traits at young and older ages. In softwood species, juvenile wood specific gravity is correlated positively and strongly with that of mature wood and whole trees, even when juvenile wood specific gravity is measured only a few years after planting (Lowe and Byram 1995). The situation is somewhat different in poplars, but several authors have addressed the issue. In Populus tremuloides, e.g., Yanchuk et al. (1983) 
found that specific gravity was moderate near the pith, declined gradually through roughly the tenth annual ring, and then rose again across subsequent rings. Herpka (1990) documented a similar pattern in poplar species and hybrids, finding also that specific gravity at two or four years of age was moderately to strongly predictive of the trait at later ages. Thus, poplars with desirable levels of specific gravity and/or dry wood substance production can be selected at early ages.

\section{PERSONAL COMMUNICATIONS}

Individuals engaged in poplar breeding and related research were interviewed to gather impressions concerning feasibility of modifying feedstock quality traits via classical selection and breeding. Regardless of background, most agreed on importance of lignin and cellulose. Certain other traits, e.g., growth and adaptability, were considered more important by breeders, but quality traits seemed worth considering, if more genetic/economic information and proper incentives were provided. Commercial breeders, understandably, were more optimistic than their academic counterparts.

Breeders generally acknowledged that poplar breeding materials were not nearly so limiting as in the past. Sizable breeding populations of poplar species and/or hybrids have been or are being assembled in most regions of the United States. Rising concerns over fiber supplies have prompted forest products companies to undertake and/or accelerate efforts. The significant stimulatory role played by the Bioenergy Feedstock Development Program was applauded.

Concerning generation and harvest intervals, breeders indicated that selections, whether new ones from the wild or proven ones from past breeding efforts, can be mated within 3-10 years of have been identified. Intervals varied among regions, with those in the Pacific Northwest being shortest. These relatively short generation intervals imply considerable potential for maximizing genetic gain per unit time.

Many breeders produce in excess of 100 cross-pollinated families annually, each containing 100 or more seedling offspring. Seedlings are cloned, planted, and screened to eliminate those that do not root well, grow slowly, and/or suffer pest attack. Screening and multiplication require roughly five years, after which superior clones are moved into production. Yet higher numbers could be achieved without unduly raising costs. Such numbers seem sufficient to maintain high selection intensities and overcome any negative correlations between quality traits and productivity.

Responses to queries concerning time to harvest indicated that most breeders, regardless of product, planned on commercial harvest within 6-10 years of planting. Despite this short interval, many breeders expected to have new, improved clones available for planting the next rotation. Coppicing therefore did not figure heavily in their programs. Coupled with the modest heritabilities of lignin and cellulose contents, these characteristics of poplar breeding, testing, and utilization tend to favor selection and breeding for feedstock quality traits.

On the negative side, most breeders viewed the limited genetic variability in lignin and cellulose contents as an obstacle, one that is likely to limit extent and speed of improvement. Uncertainties about nature and strength of correlations with other traits, especially growth, were also a concern. Breeders are loath to sacrifice gain in growth, until the economic merits of adding other traits is clear. Marker and transformation specialists viewed these aspects as increasing the attractiveness of their approaches. 
Of equal or greater concern to breeders was the apparent high cost of measuring wood chemical composition. Trait measurement costs are a major contributor to overall breeding costs. In many cases, breeding and testing populations are already large, sufficiently large to support selection for additional traits. Costs of maintaining and manipulating these populations must be borne even if breeding efforts continue to involve only traits emphasized to date. Thus, adding a trait would not increase routine breeding costs per se to significant extents, but overall costs would increase in proportion to those of assessing the new trait. Costs of measuring lignin and cellulose contents have dropped dramatically in recent years, but all types of specialists, especially breeders, would prefer that they be even lower. Continued emphasis on method development and technology transfer is necessary to reduce measurement costs to acceptable levels.

Most interviewees expressed concern about unintended consequences of reducing lignin. How much can it be reduced without disturbing growth and/or increasing susceptibility to biotic and abiotic stresses? In switchgrass (Panicum virgatum), e.g., breeding to reduce lignin content, i.e., increase ruminant digestibility, has been observed to decrease not only biomass but also winter hardiness (Vogel 12/07).

Many breeders expressed equal or greater interest in adding other traits, i.e., specific gravity and cell wall thickness. This stems primarily from the impact of these traits on solid wood product quality as well as pulp production and paper quality. Indeed, improving these traits in desired directions, especially specific gravity, may pay greater dividends than reducing lignin content. Information on genetic parameters is more abundant and reliable, prospects for gain are considerable, and measurement costs are lower. In addition, such improvement could facilitate ethanol production by virtue of raising overall dry wood substance production and, potentially, the proportion of cellulose to lignin.

On the whole, breeders viewed modifying lignin and cellulose contents worthy, attainable goals and would add the traits to improvement programs if more and better genetic data and appropriate incentives were available. On the economic front, few breeders doubt value of the traits. Indeed, those working with pulp and paper companies believe that even a single percentage point reduction in lignin content would have great value and look forward to learning how that value will affect what they can afford to spend on trait measurement and breeding.

\section{SUMMARY AND CONCLUSIONS}

In the past, U.S. poplar breeders, by and large, focused on adaptability, growth, and pest/stress resistance, with lesser emphasis on other traits, especially wood properties. Much the same holds for projects supported in whole or part by the Bioenergy Feedstock Development Program. Gains have been substantial, and further improvement is anticipated(Tuskan 1998, Wright and Tuskan 1998).

Over the long run, however, productivity is but one element in the overall matrix needed to reduce ethanol production costs. Wood chemical and physical properties clearly affect process yields and efficiencies. Viewed in this more inclusive context, biofuels and related poplar breeding programs seem sufficiently mature to have more specific breeding objectives. One example of movement toward these ends is that taken by Eucalyptus breeders in Portugal (Borralho et al. 1993). Briefly stated, breeding efforts should be designed to reduce costs, promote efficiencies, and increase profitabilities at all points having leverage in the growing, harvesting, transportation, and processing chain. 
Some steps in this direction have been taken in the United States, primarily by southern pine breeders. Some years ago, gains likely to result from including specific gravity in efforts to improve dry wood weight were considered marginal (Bridgwater et al. 1983). This outlook has since changed dramatically because of increased concern over higher proportions of juvenile wood, possible declines in specific gravity caused by continued selection for growth, greater appreciation for impacts of wood properties on processes and products, and heightened awareness of trait economic values. Working with specialists familiar with the entire chain of pulp manufacture, Lowe et al. (1999) developed a selection index that incorporates genetic parameters for growth and specific gravity along with economic values associated with costs of growing, harvesting, hauling, and processing.

That similar tactics will prove useful in improving energy crops seems clear. Poplars can be bred and manipulated with relative ease, are propagated and planted clonally, and have short harvest rotations. Needed only are better information on genetic parameters and economic weights associated with the impact of various traits on the many steps in the processing chain.

So, where do prospects for modifying feedstock quality via classical selection and breeding fit within these contexts? Information on the genetics of lignin content are not especially abundant, but most breeders argue that a reasonable debate of feasibility is possible. Genetic variation is small compared to many other traits. Ranges extend from a low of 2-3 percentage points to highs of 9-10 percentage points. Heritability estimates vary, but generally are moderate to high. Correlations among ages are not viewed as restrictive, especially for short-rotation hardwoods. Genotype X environment interactions have been observed but generally are small. Correlations with growth and other currently important traits tend toward the negative, but typically are weak and variable.

Knowledge concerning the genetics of SGR is extremely limited, although some phenotypic observations suggest that lowering lignin content would raise SGR. Efforts to modify this characteristic are best deferred until more information is available not only about its genetics but also about its relevance to processing.

The story for cellulose content is similar to that for lignin, except that genetic data are even less abundant. Heritability estimates are lower and inheritance patterns seem less additive than for lignin content. On the positive side, variation is greater, and the trait tends to have a positive correlation with specific gravity.

Measurement costs, a major factor in overall costs of breeding, should no longer be viewed as an obstacle. Costs have declined greatly as a result of recent advances in measurement technology. Many breeders, however, seem unaware of these changes, and improved communication and technology transfer should diminish their concerns and sharpen their interest in breeding for reduced lignin and/or increased cellulose contents.

Economic returns from modifying feedstock quality obtained via classical selection and breeding, though uncertain, could be substantial. Even minor reductions in lignin quantity, e.g., would reduce costs of pulping and papermaking. Impacts on ethanol production probably would be lower but still substantial. Nonetheless, breeders are unlikely to add the trait to their programs until economic outcomes are quantified in forms suitable for inclusion in selection indices. Moreover, breeders are not likely to seek additional work unless appropriate incentives are provided.

Against this background, lignin content seems a trait for which improvement can be attained via classical selection and breeding. Additional information on genetic 
parameters, however, are needed. Costs of adding the trait would not be great, provided that measurement costs are reduced to acceptable levels. The limited genetic variability would restrict gain per unit time, but even nominal reductions could be profitable. More and better information on economic implications are therefore a must.

Given present knowns and unknowns, direct improvement of cellulose content seems more problematic.

As an alternative, breeding efforts might be deployed more lucratively on other traits, deemed likely to decrease lignin and increase cellulose content indirectly. Selection for increased specific gravity, e.g., stands to raise dry wood productivity.

Positive impacts would accrue in many parts of the growing, processing, and manufacturing chain, e.g., in harvesting and transportation, as well as in processing, e.g., potentially increased cellulose content and commensurately lower lignin content. This tack seems especially attractive in that it would increase desirability of poplar wood to a wider array of customers, thereby assuring growers access to several markets.
As an example, the bottom portion of trees could be used to manufacture high-value solid wood products (see Coproducts) with residues from that part and the remainder of the tree being chipped for alcohol production. From the standpoint of ethanol production alone, classical selection and breeding for dry wood production, coupled with genetic transformation to alter lignin content, seems the optimal and most feasible route.

When considered alone, modification of feedstock quality, particularly reduced lignin content, via classical selection and breeding appears feasible. Progress would be slow but steady, and the approach is more likely to prove socially acceptable than genetic transformation and more likely to yield near-term returns than marker-aided selection. Provision of additional information on genetic parameters and economic values as well as construction of effective selection indices would hasten involvement of poplar breeders. 


\section{GENETIC MARKERS AND MAPS}

Most economically important traits in forest trees vary quantitatively, rather than qualitatively, and are subject to considerable environmental influence. Tree breeders therefore estimate heritabilities, variance components, and breeding values by statistical analyses of measurements collected from large numbers of offspring in multiple, often widely separated field trials over years or decades. Decisions typically are made at rotation age, or at best midrotation. Techniques permitting evaluation at a quarter- or half-rotation have enhanced gain per unit time, but the process still requires several years, even in short-rotation species. Generation intervals of 3-10 years further lengthen the process. Research that shortens breeding cycles will increase efficiency and economic return.

In recent times, advances in molecular biology research have yielded techniques for development of genetic maps and markers. Discovery of markers that are correlated with useful traits hold much promise for improving efficiency throughout the breeding cycle (Dinus and Tuskan 1997). They theoretically could be used to select promising parents for breeding and/or valuable offspring for propagation and planting at very early ages and without lengthy field testing. Markers could also be used for fingerprinting, i.e., for confirming identity of species, hybrids, and individuals.

\section{GENETIC MARKERS}

Restriction fragment length polymorphisms (RFLP) were the first such markers developed and applied. Since then, the field has grown tremendously, and an array of marker types and techniques have become available, largely due to application of polymerase chain reaction (PCR) technology. According to Cervera et al. (1997), ideal markers have codominant inheritance (homo- and hetero-zygotic states), are selectively neutral, and are evenly distributed across genomes. The most useful techniques were described as those capable of detecting many polymorphic loci with multiple alleles, and that are highly reproducible as well as easy, fast, and inexpensive to apply.

RFLP markers are based on polymorphisms observed after DNA digestion with restriction enzymes, are codominant, distinguish multiple alleles, and can be applied across laboratories. Analyses, however, are difficult and labor intensive. They also require large quantities of DNA, and depend on availability of useful probes. Relative to other markers, RFLP are of moderate to high cost. They have been used to generate genome maps, examine architecture of traits, monitor inheritance, track introgression in backcrossing, study genetic diversity, and detect genetic homologies among species. Random amplified polymorphic DNAs (RAPD) markers are generated by PCR amplification using arbitrary, short primers to detect single nucleotide changes, deletions, and insertions. RAPD technology does not require sophisticated probes, works with small DNA amounts, can be automated, and is rather simple, fast, and inexpensive. On the down side, RAPD generally are dominant and do not differentiate between homo- and heterozygous loci. Having only dominant markers is a serious drawback in research on species as heterozygous as forest trees. In addition, reproducibility is difficult, and they tend to be laboratory specific. Even so, RAPD have been used to produce genetic maps, demonstrate correlations with important 
traits, estimate genetic variation, examine taxonomic relationships, and identify useful genes.

Simple Sequence Repeats (SSR) or microsatellites yield high levels of polymorphisms; allelic variants are abundant and widely dispersed across plant genomes. SSR markers are codominant, and provide more information than those produced by most other techniques. Another advantage, according to some investigators, is that SSR can be transferred among genotypes within species and sometimes among species. The technique, however, requires moderate amounts of DNA and some initial DNA sequence information. As a result, SSR costs are greater than for most other techniques, even though licensing fees are limited to only the PCR portion of the procedure. SSR markers have been used for mapping genomes, identifying clones and varieties, and analyzing genetic variation.

Amplified Fragment Length Polymorphism (AFLP) technology is as reliable as that for RFLP, requires simple primers, yields large numbers of mainly dominant polymorphisms, and is highly reproducible. Large numbers of loci can be examined in individual assays, but many investigators argue that the technology is difficult to apply, less easily automated, and more expensive than RAPD technology. Nonetheless, AFLP have been used to map genomes, document variability, confirm clonal identities, and identify genes associated with traits of interest. Many such applications have been made in support of poplar breeding and research programs (Cervera et al. 1997).

Other PCR-based techniques are being developed to detect multiple, codominant alleles. Some examples are Sequence Tagged Site (STS), Single Strand Conformation Polymorphisms (SSCP), Expressed Sequence Tagged Polymorphisms (ESTP), and Sequence Characterized Amplified Region (SCAR).
Large-scale research undertakings, such as the Human Genome Project, are yielding ever more sophisticated and high-throughput techniques. Single Nucleotide Polymorphisms (SNP) yield large numbers of markers, and so-called Gene Chips are facilitating automation and lowering costs while maximizing genetic information (Sederoff 09/29). These newer techniques require specific sequence data, a considerable expense, and are therefore not yet widely used in research on forest trees. Sequence data is becoming available, and these and even newer techniques will be used more widely.

The foregoing should illustrate that useful marker technologies are available, and that improvements in utility and cost can be expected. Utilization depends on tree species and application. Consideration must also be given to needs for technical personnel and facilities as well as to costs of licensing specific technologies, equipment, and reagents. The following describes the state-of-the-art for short-rotation hardwoods. Discussion centers on poplars but also incorporates results from the considerable literature on Eucalyptus and softwoods to fill gaps and reinforce critical points.

\section{GENETIC MAPS}

Construction of genetic maps for commercially important tree species, hardwoods and softwoods, is under way in a number of laboratories around the world. In general terms, the goal of map construction is to organize segregation data for individual genetic markers such that their location in the genome and linkage to one another can be understood. In addition, genetic maps provide information on how markers are distributed across genomes and extent of genome coverage. As concerns poplars, Cervera et al. (1997) indicated that two maps had been published and that at least five others were being developed at time of 
their review. Some investigators had rather specific uses in mind. As an example, early interests of European laboratories centered on identifying and mapping markers for disease resistance, e.g., Goue-Mourier et al. (1996). Of special relevance to the present report are mapping efforts of the University of Washington/Washington State University Poplar Molecular Genetics Cooperative (Bradshaw et al. 1994, Bradshaw 1996). The map is based on a three-generation Populus trichocarpa X P. deltoides pedigree. Clones of the grandparents, parents, and offspring are growing at three locations, two of which are dramatically differing environments. Initially, the map was assembled using RAPD, RFLP, and STS markers, but the latest version includes SSR markers as well. The map is of sufficient size and scope to permit identification of simple and complex genetic elements and is being used to analyze genetics of phenology, crown architecture, tree form, growth, wood quality, disease resistance, and ease of vegetative propagation, as well as to quantify genotype $\mathrm{X}$ interactions and age-age relationships.

\section{RESEARCH, DEVELOPMENT, AND APPLICATION}

Use of marker technologies in selection and breeding can be viewed as falling into several categories (Cervera et al. 1997). Short-term uses include investigating taxonomic relationships, quantifying genetic variability, and identifying genotypes as well as selecting for simple traits. Some examples of longer term applications are dissecting complex traits, integrating traits in crossing and backcrossing, selecting parents, early selection, and identifying genes as well as genetic homologies among poplar and other species.

\section{Taxonomy, Variability, and Identity}

The genus Populus represents a rich resource, with over 30 species spread across the Northern Hemisphere. Despite much research on morphological differences, crossability patterns, and other differentiating factors, taxonomic relationships and patterns of genetic variability remain unclear. Marker techniques have been and are being employed to better delineate species, characterize variability, document relatedness, and clarify hybridity (Tuskan et al. 1996, Cervera et al. 1997). Such research underscores the tremendous variability accessible to breeders via interspecific hybridization and backcrossing (Stettler et al. 1996). Markers are also being used to identify poplar genotypes, thereby facilitating execution and management of breeding programs. University of Washington/Washington State University Poplar Molecular Genetics Cooperative members, e.g., can have 10 clones fingerprinted without charge annually (Bradshaw 09/17). Since poplars are dioecious, markers for gender determination would also be helpful to breeders. Knowing gender early in the lives of prospective parents would ensure balanced numbers of males and females and reduce costs by eliminating need to preserve large numbers of unknown sex. A useful gender marker has been found in Salix viminalis (Alstrom-Rapaport et al. 1998), but further research on poplars is needed. Marker technology should also be extended to monitor potential for gene flow from transgenic trees to natural populations.

\section{Markers for Simple, Qualitative Traits}

Utility of genetic markers in breeding derives from their correlations to valuable traits. In theory, much time and expense 
could be saved by screening offspring from desirable parents for a particular marker at an early age, and then field testing only those having the marker in question. If correlations were particularly strong, offspring thus selected could be moved directly into production. In either event, years of testing large numbers of individuals with uncertain value could be avoided. Selection and breeding for simply or qualitatively inherited disease resistance exemplifies this application. Disease resistance not only is important but also is difficult and expensive to assess. Reliability of nursery and field trials can vary widely as a result of environmental and biotic factors and should, therefore, be large, replicated over several environments, and repeated in time. Though more precise, laboratory tests are costly. To offset these difficulties, considerable research has been done on markers, and several useful ones have been identified in poplars (Cervera et al. 1996, Newcombe et al. 1996). Some investigators hesitate to use such markers, as they may not work in populations other than those in which they were identified (Bradshaw 09/17). Reluctance also stems from concern that they are associated primarily with qualitative resistance which could be overcome by shifts in pathogen virulence. Development and application costs are also a concern.

\section{Markers for Complex, Quantitative Traits}

Simple Mendelian inheritance of important traits is rare in forest trees. Most traits show continuous or quantitative variation, a phenomenon assumed to result from combined effects of numerous genes, each having small effects. The same or similar outcomes, however, may also be caused by small numbers of genes having large effects, with/without remainder of the overall influence caused by others with small effects. Systems involving few genes with large effects, or groups of tightly linked genes whatever the magnitude of individual effects, are termed quantitative trait loci (QTL). Markers for QTL allow description of quantitative traits in terms of gene number, allelic variation, magnitude of effect, and mode of gene action. Recent research affirms not only that many quantitative traits are controlled, at least in part, by QTL but also that useful markers can be identified (Sewell and Neale In press).

Identification and mapping of markers associated with QTL involves assay of a segregating population produced by crossing individuals that are genetically distinct for the trait in question. Statistical analyses are then used to identify correlations between phenotypes for the traits and inheritance of individual markers in the segregating offspring. If a genetic map has also been constructed, estimates can be made of the extent to which markers cover the genome and positions of markers relative to one another within linkage groups. QTL detection depends primarily upon presence of QTL in pedigrees, how much a QTL affects the trait in question, and size of the segregating population. Trait heritability also is important; QTL are most likely to found for traits controlled by small numbers of genes with moderate to large effects and not greatly influenced by the environment.

Though simple in concept, QTL analyses and application can be difficult and costly. Consideration must be given to potential for variation in phenotypic trait expression caused by genetic background, tree age, and different environments. Reliability also demands that traits be measured on and genetically assayed in large numbers of individuals, preferably 100 or more (Bradshaw 1996). At least two shortcuts can be used to minimize this number. As one example, QTL having major effects can be identified by restricting genetic assays to individuals having extreme 
phenotypes. In clonally propagated species, multiple ramets can be assessed to increase precision of phenotypic measurements without raising numbers of genetic assays (Bradshaw and Foster 1992). This tactic is useful for traits having low heritabilities, and can also be used to quantify effects of epistasis and genotype $\mathrm{X}$ environmental interactions.

QTL analyses in short-rotation hardwoods indicate that large proportions of variation in many quantitative traits may be controlled by small numbers of genes with large effects (Bradshaw and Stettler 1995, Grattapaglia et al. 1995). Bradshaw and Stettler (1995) found that variable but small numbers of QTL explained substantial proportions of phenotypic and genetic variation in poplar phenology, stem form, and growth. Stem volume at age two was largely controlled by four QTL, which explained 49 and $86 \%$ of phenotypic and genetic variance, respectively. Three of the four exhibited dominance and the other appeared overdominant. Alleles from Populus trichocarpa were associated with increased height, while those from $P$. deltoides contributed to diameter. The authors attributed heterosis for F1 volume growth to complementary action of these dominant alleles. For some traits, QTL effects varied with family background, age, and environment, suggesting that markers then available could not be extended to other families, ages, or environments. Given these findings and reliance of many poplar breeders on heterosis from interspecific hybridization, Bradshaw (1996) speculated that clones could be designed and bred to incorporate QTL for wood quality from $P$. deltoides, tree form from $P$. trichocarpa, early spring growth from $P$. maximowiczii, and disease resistance from $P$. nigra.

Recently, Bradshaw and coworkers expanded their QTL research to include poplar wood properties. Although such properties are heritable (see Classical Selection and Breeding), Tuskan et al. (1999b) found no QTL markers for specific gravity or lignin, cellulose, and hemicellulose contents. They hypothesized that the genetic model used for QTL analyses was inadequate because each parental species was represented by a single marker, whereas each could be contributing several alleles. Results are being reevaluated with an improved model and additional SSR markers.

Mention must be made that the poplar research described above required 10 or more years and hundreds of thousands of dollars. Identifying QTL and their markers is expensive, and yet additional funding is needed for further development and transfer of technology to breeders. Moreover, uncertainties persist as to QTL stability over families and populations other than those used in development to date and across the range of tree ages and environments typical of those used in short-rotation forestry.

Heterosis, as noted above, is often an objective of poplar breeders. Since testing for heterosis is expensive, Eucalyptus breeders have evaluated genetic markers as a shortcut. They assumed that heterosis results from heterozygosity, and hypothesized that the greater the differences between parents at neutral loci, the more likelihood of obtaining heterosis. Using offspring from crosses among E. globulus provenances and RAPD markers, Vaillancourt et al. (1995) found that genetic distances between parents did not predict heterosis. The authors advanced several explanations for the negative outcome, including the possibility that heterosis springs not from heterozygosity but rather from a few QTL with multiple alleles. They also suggested that their dominant RAPD markers yielded insufficient genetic information. Verhagen et al. (1995) used RAPD markers to quantify genetic distances between E. urophylla and E. grandis parents mated to produce interspecific hybrids. Numerous markers were identified; most showed considerable genetic distances 
between the species, but none predicted hybrid vigor. Tuskan (01/07) indicated that agronomic crop breeders now hold that heterosis results from heterotic regions in genomes, and that genetic distances among parents are not useful for predicting heterosis. More research is needed if marker technologies are to facilitate this valuable poplar breeding method.

Various marker technologies are being developed to enhance efficiency of the aggressive Eucalyptus breeding program at Aracruz S.A., Brazil (see Classical Selection and Breeding). Bertolucci et al. (1995) have identified QTL explaining significant proportions of phenotypic variance in wood density. QTL influence on screened pulp yields, a proxy for holocellulose content, however, was considerably lower. Outcomes paralleled those from traditional variance analyses, i.e., wood density has a higher heritability than pulp yield. The authors plan to include marker information in selection indices to improve accuracy of and reduce generation intervals.

Also working with Eucalyptus species and hybrids in Brazil, Grattapaglia et al. (1995) concluded that pedigrees, crosses between inbred lines, so useful for marker detection in agronomic crops are not available in trees. They contended that those common in tree breeding, i.e., twogeneration pedigrees of large full- or halfsib families from crosses among heterozygous, outbreeding parents, would be viable substitutes. Accordingly, they evaluated efficacy of RAPD markers for generating genetic maps as well as detecting QTL and markers for selection. Phenotypic and genetic data were obtained from field trials of a full-sib family derived from crossing two E. grandis and E. urophylla parents and a half-sib family from the same E. grandis parent. Full-sib assays revealed a number of QTL that explained meaningful proportions of phenotypic variance in micropropagation, rooting, and coppicing abilities. Overlapping of QTL effects across species was detected as was evidence that some QTL affected more than one trait, implying that genes controlling individual traits either had pleiotrophic effects or were linked. In half-sib trials, 12 QTL explained substantial phenotypic variation in diameter growth, specific gravity, and bark weight at 6.5 years of age. Only one, however, exerted influence on pulp yield, an expected outcome given its low heritability. The authors concluded that QTL useful for tree breeders can be detected in almost any type of family, but that size of QTL effect and interactions among QTL were limiting factors. Plans were outlined for evaluating QTL stability over genetic backgrounds, ages, and environments.

According to Grattapaglia (in press), SSR mapping could unify maps across species and resolve the question of stability across families. Toward these ends, Brondani et al. (1998) presented an SSR map for E. grandis and E. urophylla, claiming transportability across species as well as discovery of high levels of heterozygosity and allelic variability. Nonetheless, further research on this key issue is essential, lest current and future marker technologies remain restricted to application in the species, populations, and pedigrees in which they were developed. Until this is achieved, maps and markers will have to be generated for each family in a breeding program. Also critical is research on applicability across ages and environments as well as on economic feasibility.

Williams and Neale (1992) argued that specific gravity was an ideal trait for testing efficacy of marker-aided selection. The trait is important to most breeders and growers. Since correlations with growth are weakly negative, continued selection for growth could lower specific gravity to unacceptable levels. The trait has high heritablility, strong juvenile/mature relationships, and little genotype $\mathrm{X}$ 
environment interaction. Also, measurement is expensive; sample collection and assay costs are more than an order of magnitude greater than those of measuring growth. The authors projected that marker-aided selection could cut four years off the Pinus taeda breeding cycle.

Accordingly, efforts were launched to discover QTL and markers for specific gravity using a three-generation Pinus taeda pedigree. Early research revealed several QTL associated with the trait (Groover et al. 1994, Knott et al. 1997). Two exhibited genotype $\mathrm{X}$ interactions, and the strongest explained only $8 \%$ of phenotypic variation. In continuing research, Tuskan et al. (1999a) found two QTL that explained roughly $10 \%$ of variation in early and late wood lignin content. Two others accounted for approximately $13 \%$ of variation in earlywood glucan content. Yet others were associated with hemicellulose content. Sewell et al. (1998) confirmed the glucan results, and reported that several QTL accounted for $22 \%$ of specific gravity variation.

Using the same pedigree, Sewell et al. (in press) recently detected nine unique QTL associated with specific gravity and accounting for $5.4-15.7 \%$ of phenotypic variation. These proportions were considered small and probably overestimated as a result of pedigree size and nature. Several others were identified that were associated with specific gravity of individual annual rings. Most were specific to either earlywood or latewood. A number of QTL, particularly for earlywood specific gravity, were associated with the trait in all annual rings, inferring consistent influence across growing seasons, and therefore likely to have sound value for breeders. Some, however, appeared influential only in early or later years, suggesting that further analyses of temporal variation could help explain developmental factors affecting juvenile to mature wood transition. Evidence was also presented that indicated most QTL exhibited additive gene action, a finding in line with its high heritability. Tuskan et al. (1999a) and Sewell et al. (in press) concluded that, if verified in another pedigree, their QTL explained sufficient phenotypic variation to warrant testing marker-aided selection as an aid to breeding for improved wood properties. Sewell et al. (in press) indicated that verification experiments are in progress.

\section{PROBLEMS AND PROSPECTS}

Various marker technologies have been and/or are being utilized in tree breeding. Several are helping to manage and execute breeding and production programs, e.g., university/ industry/ government cooperatives help poplar breeders determine and maintain clonal identity. Substantial progress has also been made in map construction and QTL identification, especially in Pinus taeda, Eucalyptus species, and poplars.

As concerns marker-aided selection, however, more questions than answers are apparent. Challenges to application in tree breeding include not only those facing agronomic crop breeders but also others unique to trees (Strauss et al. 1992).

Linkage equilibrium and high heterozygosity are common. Most important traits are quantitative and have modest heritabilities, weak age/age correlations, and often substantial genotype $\mathrm{X}$ environment interactions. Finally, most tree breeding enterprises are designed to accumulate favorable alleles via recurrent mating and selection in large populations.

High heterozygosity has several implications for marker development and application, not least of which is the impact of differing genetic backgrounds. The danger is that QTL expression in offspring of a cross may differ dramatically from that in offspring derived from crossing one of the same parents with yet others. Problems of variable responses have been noted even 
in highly domesticated crops. Beavis et al. (1991), e.g., reported that few QTL associated with Zea mays height growth were common across $\mathrm{F} 2$ populations produced from inbred lines. In a recent review, Sewell and Neale (in press) acknowledge problems posed by inconsistency across genetic backgrounds and stress that QTL must be verified in diverse genetic backgrounds before they can be used effectively by breeders. They argued, however, that techniques for QTL detection have improved and can now be extended to developing consensus maps for families, populations, and species. As evidence, they cited recent research on Eucalyptus species showing that QTL for similar traits map to the same linkage groups and that QTL for correlated traits map to similar locations in different genetic backgrounds.

Inconsistencies across ages also are a concern. As noted above, Bradshaw and Stettler (1995) reported variation in strength of QTL associations with age in poplar. On the other hand, Campinhos et al. (1996) reported that QTL expression in Eucalyptus grandis was stable at early ages. Some age/age interaction is expected, in that many traits, e.g., height growth, exhibit weak phenotypic and genetic correlations among ages. Such interactions may not be all that problematical in short-rotation forestry, but the issue should be clarified if breeders are to develop confidence in marker applications. In connection with inconsistencies among both ages and environments, Sewell and Neale (in press) note that the more sophisticated marker technologies now available can and will be used not only to clarify such issues but also to capitalize on them. That is, QTL analyses can be used for so-called trait dissection and characterization of genetic architecture. As an example, they cited recent research on QTL affecting specific gravity in Pinus taeda (Sewell et al. in press). A number of QTL were detected that explained substantial phenotypic variation in early versus late wood and that affected juvenile wood but not mature wood. The authors argued that further QTL analyses will contribute toward understanding of factors and processes underlying development both within and across growing seasons. They also acknowledged the need for accelerated research on genotype $\mathrm{X}$ environment interactions. In the meantime, any QTL consistent across not only genetic backgrounds but also ages and environments should be tested for use by breeders.

Sewell and Neale (in press) further contend that one of the most helpful outcomes of marker research and development will be minimizing the genetic distance between markers and QTL and eventually identifying candidate or potential candidate genes. The gene itself could then be used as a marker, thereby increasing probability of identifying individuals possessing it, even if from different genetic backgrounds, ages, and/or environments. This would also permit studying not only the genetics but also the biochemistry and physiology of the trait and perhaps allow isolation of the gene for transfer into other desirable trees. The authors described several approaches for achieving these ends. Given the size and complexity of most tree genomes, they recommended a combination of techniques, since gene discovery methods used in agriculture will not be effective in trees. Along these lines, Sterky et al. (1998) isolated numerous Expressed Sequence Tags from poplars, a number of which appeared to be involved in lignin and cellulose biosynthesis. Research on this front seems of high priority, since it will benefit both classical selection and breeding and genetic transformation.

Costs of developing and applying marker technologies must also be addressed. Assembling pedigrees required for genetic analyses of trees is costly, and much marker research has been done on a retrospective 
basis, often with pedigrees not all that well suited. In consequence, relatively small ranges of genetic backgrounds have been used, and the precision with which QTL number and effect has been restricted. Grattapaglia (09/08) has argued that proportions of phenotypic variation explained by QTL, as given in the literature, are overestimated. Sewell and Neale (in press) agreed, and also suggested that QTL numbers have been underestimated. Remedying these problems could be costly, as larger and more suitable pedigrees will be needed. In sexually propagated species, every tree used to measure phenotypic variation represents a unique genotype to analyze. Problems can be somewhat muted in clonally propagated species, e.g., poplars, since precision of phenotypic measurements can be increased by assaying ramets within clones. Since numbers of genetic analyses do not increase in parallel, precision can be raised without large cost increases. In addition, clonal tests can be designed to permit assay of genotype $\mathrm{X}$ environment and planting year interactions as well as epistatic effects (Bradshaw and Foster 1992). In any event, either better technologies that make use of available pedigrees must be developed or greater financial resources must be allocated to assemble pedigrees of appropriate nature and size. Building this infrastructure could divert funding from breeding unless additional resources are made available. Returns on investment in marker technologies have also been questioned. To address this issue, van Buijtenen (1997) performed a series of simulation analyses in which classical selection and several forms of marker selection for wood properties were compared for a typical Pinus taeda breeding program. Costs of marker use were $\$ 100,000$ per year for 15 years. Comparing costs and returns from classical, marker only, and marker index selection showed that classical selection gave the highest benefit/cost ratios. Marker index selection produced greater genetic gains, but high costs reduced its benefit/cost ratio. Deploying funds required for marker application to raise breeding population size and selection intensity gave a benefit/cost ratio exceeding that for marker index selection. Comparisons of using markers for early selection to classical selection showed that the latter approach gave greater gains but a lower benefit/cost ratio regardless of heritability. Nonetheless, marker only selection had to provide at least a 15 year time advantage to produce equal or better discounted benefits and a 25- to 30-year advantage to yield a higher benefit/cost ratio. Simulations for traits especially expensive to measure, e.g., fiber dimensions, indicated that marker only selection was advantageous. Though not performed, simulations for wood chemical properties, e.g., lignin content, conceivably could yield similar outcomes. For clonally propagated species, marker only selection was profitable by virtue of saving time and closely matching wood properties to products.

Along similar lines, Johnson et al. (unpublished) simulated feasibility of using marker-aided selection to improve growth, tree form, and specific gravity of

Pseudotsuga menziesii. Assumptions included trait heritabilities of $0.25,0.25$, and 0.45 , respectively, 50 -year rotations, and restriction of markers to use within families for the foreseeable future. Results indicated that gains from marker-aided selection relative to those from classical selection varied with family size, magnitude of QTL effects, and land area over which improved materials were planted. Imprecise measurement of QTL as a result of limited family sizes was a particular concern. Windows of profitability were so narrow that the authors argued that more and better markers must be available to estimate rates of return with reasonable accuracy. They 
also advocated increases in family sizes, even if future research reduces marker costs.

In addressing rates of return in clonally propagated species, Teasdale (1995) projected that profitability of marker applications would vary directly with the land area over which improved clones are planted and with their useful lifetimes.

As noted above, technical difficulties, e.g., restriction to use only for within-family selection, suggest that marker-aided selection is not yet sufficiently developed for use in tree breeding. The foregoing further indicates that factors affecting economic returns, e.g., costs of development and application, time savings and genetic gains, clone/family numbers and useful lifetimes, and extent of use, also figure in determining utility. All considerations, technical and economic, indicate that efficiency of marker-aided selection must increase substantially before it will be accepted and applied by tree breeders.

\section{PERSONAL COMMUNICATIONS}

Views concerning utility of genetic marker and map technologies for improving feedstock quality varied widely. Most genetic transformation specialists were not familiar with applications beyond their own needs and were insensitive to cost. They also regarded, as feasible, using markers to verify fidelity of cell and tissue cultures, confirming presence of transgenes, and monitoring potential flow of transgenes into natural populations. Such applications are not unlike those in agronomic crops

Breeders, in contrast, focused on marker-aided selection and were quite to the point in their remarks. Most argued that the technology is not ready, and that serious efforts to undertake application, except in certain situations, would be premature.

Routine use awaits markers that are generalizable across pedigrees, environments, ages, and generations. Most foresaw first utilization in poplar breeding programs involving interspecific hybridization and intensive within-family selection. Marker specialists viewed poplars as a "test bed," but some cautioned that commercial use would not be profitable unless large numbers of clones are planted over large land areas.

As for immediate application, most interviewees agreed that markers are useful for confirming clone identities and safeguarding proprietary germ plasm. Several breeding and marker specialists from large forest products firms also indicated that markers are or are about to be used to facilitate selection for certain "niche" traits, e.g., disease resistance and high value wood properties that are costly to measure.

Breeders, interestingly, were much more sensitive about costs of marker development and application than about those for transformation. Marker costs were seen as large relative to prospective returns, and concern was expressed about reallocation of resources from breeding to marker projects. In contrast, transformation was viewed as more complementary to, than competitive with, breeding. Marker specialists generally acknowledged the need for new techniques that would cheapen both research and application and emphasized approaches that would facilitate generalization. Imprecise QTL estimates were acknowledged; marker specialists are seeking inexpensive solutions.

Industrial marker specialists, by and large, were optimistic and expected that marker-aided selection would be applied within five years, that cheaper and better techniques will become available, and that competition among emerging service laboratories will help reduce costs.

Given a choice, many interviewees would prefer to channel research from markers to identifying specific genes. Breeders view such research as providing means for achieving accurate and efficient selection across genetic backgrounds, ages, 
environments, and generations. Marker specialists agreed but foresaw other meaningful uses as well.

A few marker specialists, who were alert to recent research on transformation and mutants, viewed lignin biosynthesis as highly variable and plastic. They volunteered the view that markers can be found for and used to alter lignin content.

Breeding and marker specialists agreed that verification of marker utility is essential. Tests in different pedigrees are satisfactory for the near-term, but most would prefer results from trials spanning multiple pedigrees, ages, and environments.

Regardless of employer, breeders viewed markers as best applied by university or other cooperative and/or private service laboratory firms. Only those representing the largest private companies foresaw their organizations performing such activities. Marker specialists agreed with the service laboratory, cooperative or private, approach. The few transformation specialists who had thought about technology transfer had similar views.

On average, interviewees regarded genetic marker and map technologies as having limited practical value in the immediate future. Several short-term marker uses were viewed as viable, but marker-aided selection was considered a technology of the future.

\section{SUMMARY AND CONCLUSIONS}

In recent decades, diverse technologies have been developed for detecting and characterizing genetic variability in forest trees. Various DNA markers are being used to help manage breeding and production populations; future research should extend such markers to gender determination and monitoring gene flow from transgenic trees to natural populations. Investigations in poplar and other short-rotation species have also provided useful information about genomic structure and organization. Technologies and genetic maps now available or being developed will facilitate detection of QTL, set the stage for marker-aided selection, and foster study and isolation of specific genes.

Deciding whether and which marker technologies to use depends not only on advantages and disadvantages of individual approaches but also on costs of adding the effort relative to economic returns received from increases in genetic gain per unit time. In the near term, marker-aided selection could prove useful for improving traits that are simply inherited, have high value, and are difficult to measure, e.g., disease resistance. Over the longer term, however, advocates must demonstrate that markeraided selection increases accuracy of selection for quantitative traits and saves time and money. Decisions seem best made on a case by case basis, i.e., marker-aided selection will prove profitable only for certain kinds of traits and under specific conditions (Sewell and Neale, in press).

On the one hand, marker-aided selection does not seem useful for traits that can be improved with ease via classical selection and breeding, e.g., volume growth in Eucalyptus species. Genetic variation is abundant, heritabilities are high, juvenile/mature correlations are strong, and rotations are short. In this instance, substantial gain can be achieved via classical breeding without great expense. Since time savings will be small, markeraided selection, given its cost, is unlikely to produce acceptable returns.

On the other hand, high cost of marker technologies may be justified for traits that are difficult or expensive to measure and have high value or contribute greatly to cost control, even if they have high heritabilities, strong age/age correlations, little genotype $\mathrm{X}$ environment interaction. Time savings, under these circumstances, would be a bonus if accuracy and cost of selection were improved. Outcomes would be especially 
favorable if economic impacts of improvements on the many steps in production and manufacturing were known and could be used to construct selection indices.

Unfavorable returns can be expected from marker applications to traits having low heritabilities and/or low value. QTL analyses, however, could dissect such traits into their components, some of which could have higher heritabilities. Markers could then be used for those components provided gains in value and time were useful.

Where do feedstock quality traits, e.g., lignin content, lie on the gradient between these extremes? Genetic information for lignin content is not especially abundant (see Classical Selection and Breeding) but seems sufficient for debate of feasibility. Variation in the trait is small, ranging from 2-10 percentage points. Relatively few heritability estimates are available, but are mostly modest to high. Little information is available on other genetic parameters, but age/age correlations probably are adequate, especially in shortrotation hardwoods. Small genotype X environment interactions have been observed. Correlations with growth tend to be negative, though weak and variable.

Lignin content has been expensive to measure. Sample collection costs are on a par with those for other wood properties, e.g., specific gravity, but traditional laboratory assays cost several hundreds of dollars per sample (see Measurement of Feedstock Quality). Costs have fallen as a result of advances in instrumentation and methods; costs for pyMBMS assays are $\$ 15$ per sample. Value is difficult to pinpoint. Even a single percentage point reduction in lignin content would yield substantial costs savings in the paper industry. Impacts on ethanol processing, though uncertain, should be meaningful.

Against this background, lignin content seems a trait for which marker-aided selection could be justified. Additional information on classical genetic parameters, QTL number and strength, and economic impact would facilitate decisions. Although lagging behind that on some other wood properties, e.g., specific gravity, QTL research is under way in poplars, and definitive data should be available in a year or so. Similar prognostications could be shared for cellulose and hemicellulose contents and specific gravity, but one example should illustrate the point. Suffice it to say that QTL have been found for cellulose content in Pinus taeda and Eucalyptus species, although represented by a proxy, pulp yield, in the latter case. Research on QTL for specific gravity is well-advanced in Pinus taeda, and markers already are being deployed in Eucalyptus breeding.

Of immediate need then is continued research on poplar QTL identification and dissection. Future experiments seem best one with diverse genetic backgrounds and large sample sizes, with clonal replication across multiple locations. Attention should also be given to developing consensus maps so as to ensure information transfer across families, populations, and species. Investigations to identify candidate genes, and elucidate the basis of heterosis are also critical.

Financial requirements for developing genetic markers equate essentially to the infrastructure of a full-fledged breeding program. Additional costs will be incurred for implementation and monitoring. Proving benefits of such investments will be difficult. Expectations should not be overstated, and hard experimental data must be accumulated on realized biological outcomes and economic gains. Validation of marker research and application should have high priority and preferably involve paired comparison trials spanning most or all of a harvest rotation.

Even faster development of marker and mapping technology should occur in the 
future as spin-offs from the Human Genome and Arabidopsis thaliana projects. Spread of these technologies could be accompanied by creation of more numerous and sophisticated university/industry cooperatives and/or privately owned service laboratories. Such organizations, at first, probably will focus on aiding breeders with program execution and management. With time, however, they could expand to applying maps and markers to help breeders with selection of new parents and early selection within families. If these activities prove profitable, more sophisticated applications could follow. Fostering expansion of such enterprises could prove worthwhile.

Finally, many classical and molecular geneticists use poplars as model species. This virtually ensures rapid progress, and the BFDP can look forward to exploiting the outcomes, particularly if it provides judicious, balanced support to the most critical research, development, and technology transfer endeavors.

\section{GENETIC TRANSFORMATION}

Numerous analysts have argued that modifying lignin and cellulose contents via genetic transformation is both desirable and possible. As long ago as 1988, altering softwood lignin to be more like that of hardwoods was estimated as saving \$U.S. 6 billion in pulping chemical costs alone (Timmis and Trotter 1989). Reduced lignin content was projected to yield similar savings. Lower lignin and/or higher cellulose contents are also likely to improve ethanol production efficiency. No wonder that a host of investigators in the United States and offshore are pursuing aggressive research on this front.

Genetic transformation can bring a variety of benefits to tree improvement. Should genes for a desired trait not exist in the species of interest, they can be procured from other organisms and transferred into trees slated for breeding and/or planting. Thus, several organizations are inserting genes from hardwoods into softwoods so that both syringyl and guaiacyl lignin (Fig. 2) will be synthesized, the goal, as noted above, being improved softwood pulping efficiency. Transformation can also be used to alter expression of native genes, i.e., to increase or decrease activity of desirable genes. This involves isolating the genetic system in question, copying genes and controlling elements (promoters), synthesizing genetic constructs that enhance (sense) or obstruct (antisense) expression of native genes, and then inserting appropriate constructs into otherwise improved trees. Accordingly, antisense constructs are being used to suppress expression of genes affecting lignin biosynthesis, and sense constructs to enhance cellulose biosynthesis. By virtue of cosuppression, sense constructs are sometimes used to reduce activity of target genes. Not to be overlooked is that transformation can save time via bypassing the sexual cycle and often long generation intervals. Gene transfer should, thus, be viewed as complementing, rather than competing with, classical selection and breeding.

Ideally, traits considered for change via transformation should have high economic value, be modified such that improvement is large relative to that attainable by other methods, be the product of a reasonably well understood 


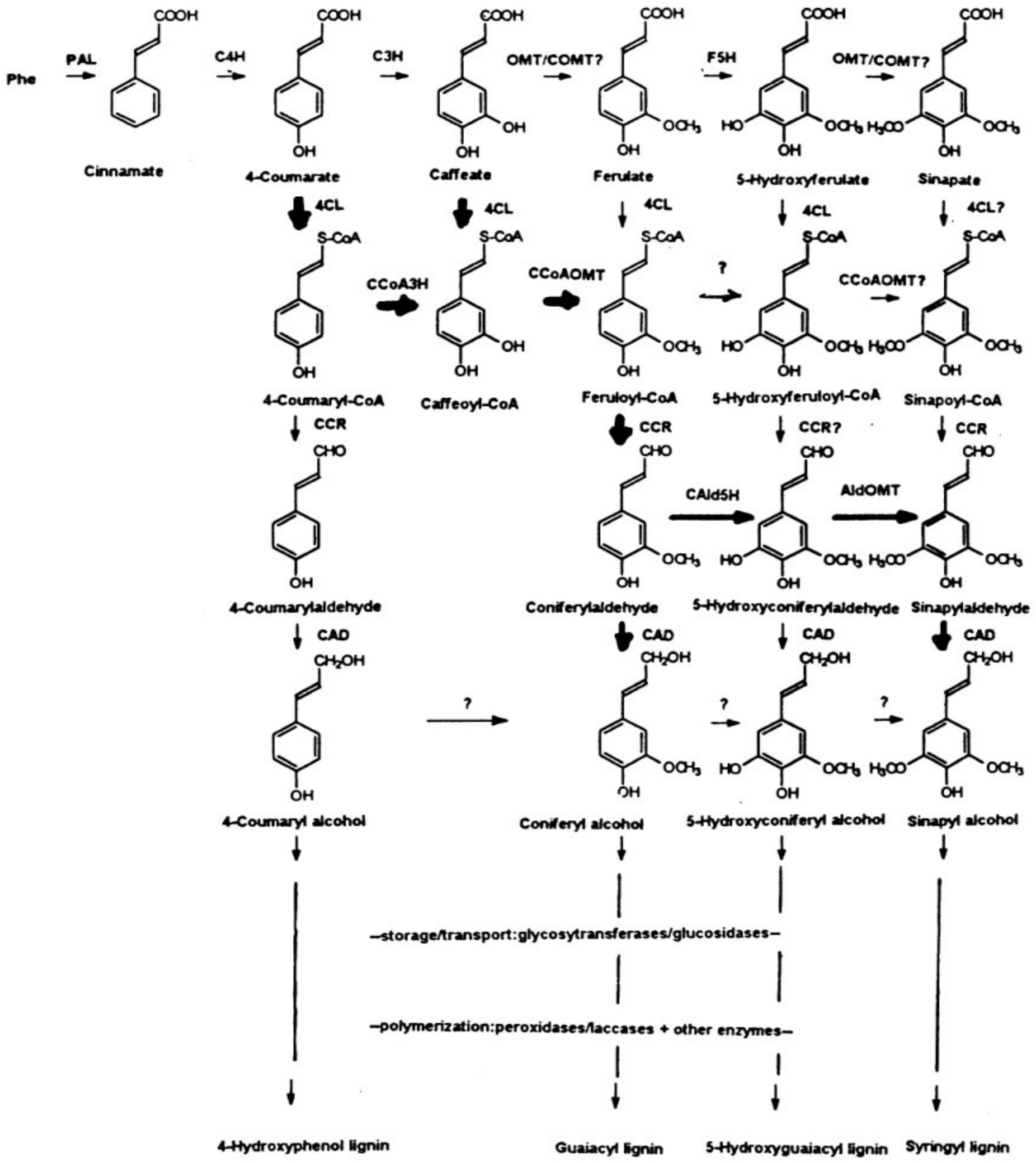

Fig. 2. Generalized diagram of the hardwood lignin biosynthetic pathway. Enzymes are indicated for each step: PAL, phenylalanine ammonia lyase; $\mathrm{C} 4 \mathrm{H}$, cinnamate 4-hydroxylase; $\mathrm{C} 3 \mathrm{H}$, 4coumarate 3-hydroxylase; OMT, O-methyltransferase; COMT, caffeate O-methyltransferase; F5H, ferulate 5-hydroxylase; 4C1, 4-coumarate CoA ligase; $\mathrm{CCoA3H}$, coumaroyl CoA 3-hydroxylase; CCoAOMT, caffeoyl CoA O-methyltransferase; CCR, cinnamoyl-CoA reductase, CAld5H, coniferyl aldehyde 5hydroxylase; AldOMT, 5-hydroxyconiferyl aldehyde O-methyltransferase; CAD, cinnamyl alcohol dehydrogenase; glucosyltransferases; glocosidases; peroxidases; laccases; and other oxidases. Adapted from Campbell and Sederoff (1996) and Hu et al. (1999). Cofactors are not shown in order to simplify the diagram. Uncertain enzymatic steps are labeled with question marks. Bold arrows indicate the primary path for biosynthesis of the syringyl and guaiacyl residues that predominate in hardwood lignin as advanced by Li et al. (2000). 
biochemical pathway, and be controlled by one or a family of related genes. Moreover, modification should not adversely affect survival and growth. Lignin content meets these criteria rather well, although information is limited on survival and growth. Moreover, lignin cannot be converted directly to fermentable sugars and has few uses beyond process fuel. As a result, advocates of genetically modifying feedstock quality generally have favored reducing lignin content. Raising carbohydrate content was once considered difficult (Dinus et al.1990). Biosynthetic pathways were viewed as more complicated, with greater potential for adverse side effects, than that for lignin. This view has changed; research on modifying cellulose biosynthesis is aggressive, and results are also included.

Several methods are used for poplar transformation, and a number of genes have been inserted and expressed in various species and hybrids. The first such achievement involved a gene conferring herbicide tolerance (Fillatti et al. 1987). Indeed, poplars are now regarded as a model system for transformation as a result of the ease with which many can be manipulated in and regenerated from cell and tissue cultures (Chandler 1995, Dinus and Tuskan 1997). Improvements in transformation efficiency and extension to wider arrays of genotypes, however, remain important research needs (Han et al. 1996, Dinus and Tuskan 1997). Transforming and regenerating the many genotypes used in commercial breeding and planting programs represents a formidable task.

Against this background, the following highlights the lignin biosynthetic pathway possible points for intervention via transformation, and the outcome of attempts to change lignin quantity and composition. Information from recent attempts to modify cellulose biosynthesis is also presented. Findings from poplar research are emphasized, but some results from other species are also included. Thus, the following sections are highly targeted and should not be considered an exhaustive summary of old and new literature on modification of lignin in multiple plant species.

\section{LIGNIN BIOSYNTHESIS}

The lignin biosynthetic pathway (Fig. 2) is fairly complicated but short. Basic features have been known for some years (Campbell and Sederoff 1996). Changes in our understanding of it have been frequent, especially in recent times, as genetic transformation has become a major investigative tool. Phenylalanine, an aromatic amino acid produced via the well documented shikimic acid pathway, generally is regarded as the starting point for lignin biosynthesis. This compound is deaminated by the enzyme, phenylalanine ammonia lyase (PAL) (Fig. 2).

In a series of subsequent reactions, aromatic nuclei are hydroxylated first at $\mathrm{C} 4$ (4-coumarate) and then at C3 (caffeate). As outlined in Fig. 2, 4-coumarate and caffeate are then further transformed to give the 4-hydroxyphenyl monomeric precursors in grasses and hardwoods as well as the guaiacyl monomers in all higher plants.

Hardwood lignin contains both syringyl and guaiacyl monomers. Formation of syringyl monomers involves C5 hydroxylation and subsequent methylation (Fig. 2). The means by which 4-coumarate, caffeate, and/or ferulate are converted to syringyl intermediates, however, has been the subject of much debate and research in recent years. Enzymes implicated at one time or another in these interconversions include: O-methyltransferases (OMT), ferulate 5-hydroxylase ( $\mathrm{F} 5 \mathrm{H})$, caffeate O-methyltransferase (COMT), 4-coumarate CoA ligase (4CL), coumaroyl CoA 3-hydroxylase (CCoA3H), caffeoyl CoA O-methyltransferase (CCoAOMT), and 
cinnamoyl-CoA reductase (CCR). Recent findings (Hu et al. 1999, Li et al. 1999, Li et al. 2000) indicate, however, that hydroxylation and methylation occur at other than the acid precursor level in hardwoods, with transformations occurring as indicated by the heavy arrows in Fig. 2 . Different enzymes and routes may be involved in other angiosperms, e.g., an Arabidopsis thaliana mutant deficient in F5H has been found to produce virtually no syringyl monomers (Marita et al. 1999). The final step in monomer synthesis involves conversion of coniferyl and sinapyl aldehydes to their respective acids by cinnamyl alcohol dehydrogenase (CAD).

Once the primary precursors of hardwood lignin, coniferyl and sinapyl alcohols, are synthesized, they must be moved to appropriate sites for polymerization or transported to storage sites and then remobilized. Most investigators agree that the alcohols are glycosylated to coniferin and syringin for transport and remobilization. Glucosyltransferases and glucosidases are involved in these functions, but their roles are not well understood (Campbell and Sederoff 1996). Given further information, modifying these functions, however, would seem rather precise ways to reduce lignification.

According to older reports, the last step catalyzed by enzymes in lignin biosynthesis is dehydrogenation of synapyl and coniferyl alcohols to their phenoxy radicals by peroxidases. The radicals are thought to then couple "randomly" without aid of other enzymes. Much controversy exists in this area, as some investigators argue that laccases (Dean and Eriksson 1992) and/or other oxidases (Savidge and Udagama-Randeniya 1992) are also involved, either alone or with peroxidases. Some investigators further contend that polymerization is not "random" but occurs in accordance with a template established by cell wall proteins, other spatial and temporal factors, and interactions with enzymes affecting linkages to carbohydrates. Just how polymerization occurs remains conjectural. Nature and frequency of linkages to carbohydrates are likewise uncertain.

Scientists investigating prospects for modification via genetic transformation have concluded that lignin quantity/quality can be regulated by levels and proportions of precursors available for polymerization. Decisions on intervention are made against this background, with consideration given to other factors as well. For example, intervention using antisense constructs to reduce PAL activity would seem certain to reduce precursor abundance, but intervening this early in the pathway could also affect synthesis of other metabolites. Actually, steps through formation of the thioesters via $4 \mathrm{CL}$ action are known to provide intermediates for biosynthesis of flavonoids and other compounds that function in other essential metabolic processes. In consequence, most attempts to modify hardwood lignin biosynthesis have focused on enzymes involved in transformations later in the pathway. Suppressing CAD activity, e.g., has been viewed a potent means for reducing coniferyl alcohol supplies with minimal side effects. Another possibility is regulation of lignin quality by using antisense and sense constructs to increase synthesis of sinapyl at expense of coniferyl alcohol. This approach could be used to raise SGR, should this characteristic be found to improve ethanol conversion efficiency.

\section{LIGNIN: RESULTS FROM TRANSFORMATION RESEARCH}

Findings from genetic modifications such as those mentioned above as well as others are discussed below, with emphasis on intervention at points not likely to affect functions other than lignification. Results are summarized, primarily for poplars, by 
enzyme, in order from beginning to end of the biosynthetic pathway. Some experiments have produced results useful for application in ethanol conversion, while others have yielded unexpected outcomes. All, however, have expanded our knowledge of the lignin biosynthetic pathway.

\section{PAL}

Some research on manipulating this enzyme in trees was performed in early years. Most investigators, however, switched emphasis to other enzymes due to concern over pleiotrophic effects. In addition, many different forms of PAL exist, and determining which is active when and where is difficult (Campbell and Sederoff 1996). Bate et al. (1994), however, used antisense constructs to modify PAL activity levels in tobacco (Nicotiana tabacum). Plants with varying degrees of PAL activity were produced. Even at severe levels of suppression, phenotypes and flavonoid synthesis were normal. Lignin quantity, however, was reduced, presumably by the flavonoid pathway being more competitive than that leading to formation of lignin precursors. Sewalt et al. (1997b) found that PAL suppression reduced lignin content in tobacco but also noted increased SGR.

\section{OMT/COMT}

Enzymes of this type are thought to catalyze the ortho-methylation of caffeate to ferulate and 5-hydroxyferulate to sinapate in some plants and have also been implicated in methylation of other intermediates (Fig. 2). Dwivedi et al. (1994) transferred antisense constructs for an OMT specific to Populus tremuloides into tobacco. OMT activity was suppressed in stems by an average of $29 \%$, and syringyl content was somewhat reduced. Using sense and antisense constructs of varying lengths, Rossitza et al. (1995) transformed tobacco and evaluated responses in terms of lignin content and composition. Response varied with construct length; constructs equal to full length of the gene were most effective. Insertions proved stable and were transmitted to offspring as a Mendelian trait. Sense constructs increased OMT activity by 170 to $370 \%$ of nontransformed controls but did not affect lignin quantitatively or qualitatively. Few instances of cosuppression were observed. Antisense constructs suppressed OMT activity but did not affect lignin content. Composition, however, was modified; SGR were lowered and an unusual lignin precursor, 5hydroxyconiferyl alcohol, was produced. The authors inferred that lignin content would be reduced only when antisense constructs reduced OMT activity by $80 \%$ or more. They added that the OMT catalyst used in their research did not methylate caffeate efficiently and suggested that COMT would be more effective.

Doorsselaere et al. (1995) produced four hydrid poplar clones with reduced COMT activity via transformation with an antisense COMT construct. None exhibited reduced lignin content. Severely suppressed clones, however, had lower SGR, a result of decreased syringyl and increased guaiacyl contents. In addition, a novel lignin monomer, 5-hydroxyguaiacyl, was produced, presumably the same compound described by Rossitza et al. (1995) in tobacco. Xylem was rose colored, a phenotype resembling those of bmr3 and 4 maize mutants. The authors expressed concern that several COMT forms exist in trees, with temporal and spatial expression varying in unknown ways, and noted that COMT antisense constructs have reduced lignin content in very few plant species.

After transforming (Populus tremuloides) with a COMT sense construct, Tsai et al. (1998) found increased activity in leaves, but lower activity (cosuppression) in stems. Lignin content was not affected, but SGR were decreased, red coloration was induced, and synthesis of a 5-hydroxyl 
guaiacyl monomer, as noted above, was observed. The red xylem coloration was attributed to incorporation of coniferyl aldehyde in the lignin polymer.

Lapierre et al. (1999) analyzed the same or similar hybrid poplars described by Doorsselaere et al. (1995) after two growing seasons. Lignin content of transgenic trees did not differ from controls, but an increased frequency of guaiacyl units as well as C5-C5 biphenyl linkages was found. The lignin was more condensed and proved more resistant to kraft pulping. The authors speculated that intervention at other points in the pathway, e.g., suppression of 4CL activity, might be more productive. Boerjan et al. (1999) confirmed these results and indicated that field trials are being continued.

Osakabe et al. (1999) addressed the question of how guaiacyl intermediates are hydroxylated and methylated to form syringyl monomers in hardwoods. They provided evidence explaining some of the aforementioned outcomes and clarifying other questions about the pathway. According to these authors, the main route to synthesis of syringyl units does not involve conversion of ferulate to 5hydroxyferulate, followed by methylation to sinapate and reduction to sinapyl alcohol. Instead, the preferred route occurs later in the pathway (Fig. 2), with coniferyl aldehyde being converted by coniferyl aldehyde 5-hydroxylase (CAld5H) to 5-hydroxyconiferyl aldehyde which is then methylated by COMT to form sinapyl aldehyde. Li et al. (2000) confirmed these findings, and further demonstrated that a specific enzyme, 5-hydroxyconiferyl aldehyde O-methyltransferase (AldOMT), rather than COMT, is responsible for methylation.

\section{CL}

In angiosperms, this enzyme catalyzes conversion of 4-coumarate, caffeate, ferulate, 5-hydroxyferulate, and sinapate acids to their respective thioesters. In early research with Pinus taeda, Voo et al. (1995) disclosed evidence that 4CL had the same form in normal and compression wood and suggested that only one gene was involved. Zhang and Chiang (1997) subsequently suggested that two nonalleleic genes were responsible for $4 \mathrm{CL}$ production and that at least one of them was expressed in developing xylem. They further noted that substrate specificity and activity of one enzyme form varied between normal and compression wood.

Hu et al. (1998) discovered two functionally and structurally distinct $4 \mathrm{Cl}$ genes, Pt4CL1 and Pt 4CL2, in aspen. Pt4CL2 was expressed in leaf and stem epidermal layers, suggesting involvement in synthesis of intermediates for production of flavonoids and phenolics, i.e., functions other than wood formation. In contrast, Pt4CL1 activity was specific to lignifying xylem tissues, implying more or less specific involvement in lignin biosynthesis. Pt4CL1 was considered a good target for genetic modification.

Transformation with antisense constructs for Pt4CL1 yielded aspen trees with up to $45 \%$ reduction in lignin content and $15 \%$ increase in cellulose content relative to controls (Hu et al. 1999). Neither lignin properties nor structure were changed, as is so often observed when other enzymes are manipulated. Also important were changes in hemicellulose composition. Xylose contents were unchanged, but those of arabinose (C5), galactose (C6), and rhamnose (C6) were increased, and the galactose/arabinose ratio was increased. Transgenic plants are growing faster than controls but are morphologically and anatomically normal. Cellulose synthase transcription was not increased; the higher cellulose content may have resulted from diversion of carbon to cellulose synthesis in compensation for reduced structural support associated with less lignin. Yet other 
factors could be involved, and causes for elevated cellulose synthesis are being investigated. The trees have been moved to field trials after several years of growth in greenhouse.

Echoing these findings, Douglas et al. (1999) identified four 4CL gene family members in Populus trichocarpa and its hybrid with Populus deltoides. One gene, 4CL3, is expressed in developing xylem and considered similar to the Pt4CL1 gene discovered by Hu et al. (1998) and used by $\mathrm{Hu}$ et al. (1999) to create transgenic aspen with reduced lignin content. Another gene, 4CL4, was expressed mainly in leaves and appeared related to the Pt4CL2 gene found by Hu et al. (1998). Substrate affinities of enzymes synthesized by 4CL3 and 4CL4 were similar to those reported by $\mathrm{Hu}$ et al. (1998) for Pt4CL1 and Pt4CL2, respectively. Transformation with antisense constructs yielded plants with reduced 4CL activities but that otherwise appear normal. At time of this writing, lignin quantity and composition had not been assayed (Douglas 10/13).

Manipulation of 4CL genes such as those reported by Hu et al. (1999) holds great promise for ethanol production. Especially noteworthy is that manipulating this enzyme resulted in the first significant reduction in lignin content, and that reduction was achieved without adverse effects on lignin composition and structure or tree growth. Said manipulation also increased cellulose/lignin ratios, a clear benefit to ethanol processing efficiency. Effects on hemicellulose composition are also of value, and further research on this aspect seems imperative. Finally, the results open the door to research on using 4CL genes in combination with others affecting lignin and/or cellulose biosynthesis.

\section{CCoAOMT}

This enzyme is thought to participate in conversion of caffeoyl-CoA to feruloyl-
CoA and, perhaps, 5-hydroxyferuloyl-CoA to sinapoyl-CoA. Few reports were found on modification of CCoAOMT activity in trees, but several European laboratories are contemplating or have initiated research. Boudet and Grima-Pettenati (1996) called for investigation of suppressed CCoAOMT activity alone and in combination with suppression of other genes so as to tailor lignins to specific manufacturing ends. Baucher et al. (1997) indicated that experiments with transgenic trees are in progress.

\section{CCR}

Research on this enzyme in poplars and other trees has been limited until recent years. A gene coding for CCR was isolated and cloned from Eucalyptus gunni by Lacombe et al. (1997). Expression was observed in differentiating stem and root xylem, but some expression was also found in leaves, suggesting that CCR reactions contribute to formation of products in addition to lignin.

Piquemal et al. (1998) transformed tobacco with an antisense construct for the CCR Eucalyptus gunni gene mentioned above. Lignin content and composition, growth, and other phenotypic characteristics were examined in progeny of the original transformants. Most offspring had orangebrown lignin that differed not only from controls but also from plants transformed with CAD antisense constructs. Coloration was attributed to accumulation of ferulate, sinapate, and acetosyringone in cell walls. Increased SGR were also found, primarily caused by decreased guaiacyl content. Plants with severely suppressed CCR activity had a roughly $50 \%$ reduction in lignin content, but also had reduced growth, collapsed vessels, and abnormal leaves. Those with less severely suppressed activity had normal lignin content and growth. Their lignin, however, contained fewer $\beta$-O4 linkages and slightly more carbon-carbon 
bonds than that in controls. Boerjan et al. (1999) reported ongoing evaluation of transgenic poplars with modified CCR levels. In yet more recent research on tobacco, Pilate (10/18) found reduced lignin content and higher SGR levels without growth abnormalities and plans to extend his investigations to poplar.

\section{CAD}

The earliest research on CAD manipulation was performed in tobacco and softwoods. In recent times, considerable research has been done with poplars. Given the sizable backlog of information, much of which is very similar, the following discussion is limited to poplars plus a few supporting tobacco reports.

In an early report on tobacco, Halpin et al. (1994) effectively set the stage for what has been found in poplar investigations. Transformation with CAD antisense constructs had little or no effect on lignin content but did cause several changes in composition. Transgenic plants incorporated fewer cinnamyl alcohols and more cinnamyl aldehydes into lignin than did controls, and exhibited a red-brown coloration in xylem tissues similar to that of bmr maize mutants. The modified lignin also proved more easily extracted by alkaline pulping chemicals. Transformants were otherwise normal in appearance. Boudet and Grima-Pettenati (1996) obtained similar findings in poplar. Baucher et al. $(1996,1997)$ confirmed these early results and further found that SGR were not changed, and that a number of free phenolic compounds, e.g., vanillin and syringaldehyde, accumulated in cell walls of transgenic poplars. Along similar lines, analyses of tobacco plants transformed with CAD antisense constructs confirmed increased frequency of aldehydes as opposed to alcohols in as well as less condensation of lignin (Stewart et al. 1997). These phenomena explain the greater ease with which such lignin is extracted by alkaline pulping chemicals. Changes of this type, however, are not likely to have favorable effects on ethanol conversion processes, which employ acid rather than alkaline environments. Actually, such modifications, especially those adding free compounds to cell walls, could indeed be detrimental.

Lapierre et al. (1999) provided additional data on changes in poplar lignin composition caused by CAD antisense constructs. Lignin content of 2-year-old trees was only slightly lower than that of controls. Frequency of guaiacyl units and labile ether bonds were similar to those in controls, but proportions of free phenolic groups were higher. The red-brown coloration noted above was ascribed to syringaldehyde contents greater than those of controls.

In a somewhat more positive vein, Pilate et al. (1999) inserted a CAD antisense construct into a different poplar genotype than that used in most of the aforementioned research by Baucher et al. (1996) and Lapierre et al. (1999). CAD activity was suppressed to a far greater extent, a residual activity of 2 to $3 \%$ as opposed to $20 \%$.

Noteworthy also were a 10 to $15 \%$ reduction in lignin content, lower SGR, and incorporation of various atypical compounds in the lignin.

To further complicate the CAD picture, a recent report on feeding radioactive precursors to shoots of three hardwood species suggests that syringyl alcohol can be produced by enzymatic conversion of coniferyl alcohol (Fukushima et al. 1999). Confirmation of this route plus identification of the enzyme and gene could prove useful. Knowledge of lignin biosynthesis has been enlarged greatly in recent years, but much clearly remains to be understood. 


\section{Glucosyltransferases and Glucosidases}

As described in a previous section, storage and transport of lignin monomers are required to ensure that polymerization occurs in appropriate places and times. The directed literature search conducted for this report did not yield much information on manipulation of these functions in trees. This outcome should not be interpreted to mean that advances have not occurred; personal communications suggest that information is fast becoming sufficient to support research on genetic modification.

Douglas (10/13) has identified a betaglucosidase as a potential target for manipulation. The enzyme is expressed in cell walls of Picea glauca and ostensibly is involved in removing glucose from coniferin, thereby remobilizing coniferyl alcohol for polymerization. The gene was identified, and antisense constructs were used to transform Picea glauca somatic embryos. Transgenic trees have been recovered and are being grown to sizes sufficient to determine if intervention affects lignification. Savidge and Forster (1998) have identified a coniferyl alcohol glucosyltransferase in conifers. Activity is tied to the annual cycle of cambial growth and dormancy, and also parallels seasonal variation in endogenous coniferin levels. Attempts to modify its activity are under way at the University of New Brunswick (Savidge 09/23). These seem fruitful areas of research, since intervention should have minimal side effects.

\section{Peroxidases, Laccases, and Other Oxidases}

These enzymes, or at least some of their numerous forms, presumably are involved in lignin polymerization. Boudet et al. (1995) indicated that early modifications of peroxidase activity gave variable results. Indeed, many such efforts did not affect lignification but instead produced numerous pleiotrophic effects (Campbell and Sederoff 1996). This should not be surprising given the many different forms of this enzyme. Nevertheless, research continues, at least in several European laboratories. According to Baucher et al. (1997), several xylem specific genes coding for peroxidases presumably involved in lignification have been cloned for research on transformation.

Potential roles of laccases in lignification were reviewed by Dean and Ericksson (1992), who advocated isolation and manipulation of laccase genes. Laccases have been associated with lignification in several tree species, but evidence for substantial involvement is conflicting (Boudet et al. 1995). Laccases appear to be present in the right places at the right times (Dean 09/24), but their role may be more that of controlling free radical production than being the driving force behind lignification per se.

Research on genetic modification nevertheless has been considerable. A laccase preparation was isolated from Acer pseudoplatanus that oxidized p-coumaryl, coniferyl, and sinapyl alcohols to insoluble polymers in vitro (Stejiades et al. 1992). Further evaluation led Stejiades et al. (1993) to propose that laccase catalyzes formation of oligomers from monomer precursors, and that peroxidases convert the oligomers to highly condensed lignin polymers. In subsequent research, full or partial copies of genes for laccases were isolated from Acer pseudoplatanus and Liriodendron tulipifera. These were used to synthesize sense and antisense constructs of varying sizes and inserted into Liriodendron tulipifera somatic embryos (Merkle 09/23). Sense constructs increased laccase activity in and lignin content of transgenic seedlings over those of controls. Transformants exhibited short, bushy phenotypes. Antisense constructs did not reduce lignin content, perhaps because only constructs of limited length were available. A laccase associated 
with lignification has also been identified in Pinus taeda (Bao et al. 1993). More recently, Grima-Pettenati et al. (1996) identified an enzyme, presumably a laccase, in poplar. The authors indicated that they were testing its specificity and intended to isolate and clone the gene for eventual use in antisense transformation.

On other fronts, Savidge and Udagama-Randeniya (1992) identified a coniferyl alcohol oxidase in several conifers, and have since characterized its properties (Udagama-Randeniya and Savidge 1995). Activity is localized in lignifying tissues (Savidge et al. 1998, Savidge 09/23). The authors recommended using transformation with antisense constructs for it, peroxidase, and laccase to clarify roles of these enzymes in lignification. Findings have been confirmed by a Scottish laboratory, where the research is being expanded (McDougall 1998). Against this background, modification of storage, transport, and polymerization functions seems risky unless and until roles of enzymes are clarified.

\section{CONTROL OF GENE EXPRESSION}

In some reports cited above, spatial and temporal specificities of elements controlling gene expression were not always clear. Most investigators employed constitutive promoters, e.g., the CV35S promoter or a strengthened version thereof. This seems appropriate in early phases of research; the tactic enhances probabilities of observable expression. Research must progress beyond this proof-of-principle phase, however, if outcomes are to have practical utility. In a few cases, investigators had identified and isolated xylem specific promoters, and fused them to sense/antisense for purposes of transformation.

Fortunately, a number of laboratories are devoting substantial resources to identifying promoters and to discerning mechanisms of genetic control. Examples bearing on lignin and cellulose biosynthesis include extensive resources committed to developing xylem specific promoters by Sederoff (09/29) and Loopstra (11/19). Scientists in some forest products firms hinted that promoters have high priority in their research. Nevertheless, earlier calls by Boudet et al. (1995) and Campbell and Sederoff (1996) for accelerated research on tissue and developmental stage specific promoters is well worth echoing and reinforcing.

\section{MUTANTS AFFECTING LIGNIN}

Mutants affecting lignin quantity/quality have been discovered in corn (Zea mays) and a number of other species including, most recently, Pinus taeda. The bmr mutants in corn are being used to create hybrid varieties with reduced lignin content (Keeling 09/03). All four (bmr1-4) are recessive and cause some reduction in lignin quantity. Some also affect lignin composition; bmr2, e.g., increases syringyl content in addition to lowering lignin content. Similar mutants have been found in other grain crops (Campbell and Sederoff 1996), and a mutant deficient in $\mathrm{F} 5 \mathrm{H}$ activity is known in Arabidopsis thaliana.

The null CAD mutant recently discovered in Pinus taeda (MacKay et al. 1997) is the first discovered in trees. The recessive cad-n1 allele is inherited as a simple Mendelian trait, and effectively eliminates CAD activity in the homozygous state. Unlike most transgenic trees with reduced CAD activity, cad-n1 homozygotes have lignin content slightly lower than heterozygotes or homozygous dominants (homozygous mutant $=28.9 \%$, wildtype $=$ $31.7 \%)$. Lignin composition is also affected (Ralph et al. 1997); large amounts of CAD substrate, coniferyl aldehyde, and several derivatives accumulate as free compounds in cell walls, and a novel monomer, 
dihydroconiferyl alcohol, polymerizes in lieu of coniferyl alcohol. Homozygous cadn1 plants have brownish xylem, resembling that of bmr corn mutants and some poplars containing CAD antisense constructs. Even though growth is slow, vascular function and mechanical support apparently are preserved.

These several examples serve to suggest that similar or perhaps more useful mutants can be found in poplars. Sensitive analytical methods and gene discovery techniques should help in these regards. Should useful poplar mutants be discovered, they could be used in breeding and/or transformation and perhaps as markers for early selection of low lignin parents. Finally, mutants in other species, if deemed useful, can be used to identify and isolate heterologous loci in trees.

\section{CELLULOSE: RESULTS FROM TRANSFORMATION RESEARCH}

In an earlier review of prospects for modifying feedstock quality, Dinus et al. (1990) suggested that information then available on biochemistry and genetics of enzymes catalyzing the many steps in cellulose synthesis was viewed as insufficient to support modification via genetic transformation, even though some enzymes seemed lucrative targets for intervention. Advances since that time, fortunately, have been phenomenal (Delmer and Amor 1995, Arioli et al. 1998, Delmer 1999).

Accordingly, progress has also occurred in genetic modification. A family of cellulose synthase genes have been identified in and isolated from poplar (Loopstra 1999, 11/19). Said genes have been sequenced and the sequences recorded in the Plant Gene Register. A cellulose synthase gene (celA) is being investigated by an Australian laboratory, and patents sought for its application (Ellis 09/30). Transgenic Populus tremuloides trees containing a sense construct of a celA homolog have been produced at Michigan Technological University (Chiang 09/15). The trees, whose phenotypes are normal, are growing faster than controls in greenhouse. A patent application has been filed, and publication in the scientific literature can be expected in the near future. An Israeli firm, CBD Technologies, Inc., has inserted a cellulose binding gene into several crops, including poplar trees. The firm claims transgenic trees grow faster and produce more cellulose than controls and expects to market the technology within five years.

Research on enzymes associated with UDPglucose processing has also proved lucrative. A gene for UDP-

pyrophosphorylase has been cloned, and sense constructs inserted in tobacco plants. Heightened enzyme activity and cellulose synthesis were reported (Xue et al. 1997). Analyses indicate a 30\% enhancement of cellulose content and a $20 \%$ increase in biomass (Ellis and Sutton 09/30). Research currently is focused on an improved sense construct and transformation of hybrid poplar.

In view of these findings, prospects for increasing cellulose content via genetic transformation seem almost as good as those for reducing lignin. With few exceptions, genetic interventions in lignin biosynthesis have modified composition, and changes reported to date are not likely to facilitate conversion of woody feedstocks to ethanol. The few reported reductions, however, are significant, and modifying both lignin and cellulose traits simultaneously could yield truly outstanding results.

\section{SOCIAL ACCEPTABILITY}

A major obstacle to commercialization of transgenic trees is concern over consequences of their release into the environment. This stems mainly from the possibility that transformants will spread so-called foreign genes to natural 
populations (Harcourt et al. 1995, Meilan and Strauss 1997). To minimize or avoid such risks, transgenic trees may have to be rendered sexually sterile, and noteworthy progress is being made on this front. Within 5 to 10 years, suitable genetic constructs should become available that induce stable, male and female sterility in poplars (Strauss 10/01). Placing such constructs in commercially valuable clones should facilitate obtaining regulatory approval for testing and use, should reduce any adverse environmental impacts of widespread planting, and could also yield important side benefits. That is, eliminating reproductive structures could channel larger quantities of energy, water, and nutrients to growth and wood production. Not to be overlooked, of course, is that use of sterile, genetically engineered trees is also likely to pay yet additional dividends by reducing environmental impacts, regardless of product, of growing, harvesting, and processing,

\section{PERSONAL COMMUNICATIONS}

When asked if feedstock quality can be improved via genetic transformation, specialists in both classical and molecular aspects of forest tree breeding answered clearly in the affirmative. Differences in opinion mainly concerned timing and social acceptance. Molecular geneticists, as expected, were decidedly more positive. Transgenic trees with altered lignin have been produced; the technology works and commercialization is only a matter of time, perhaps 5 to 10 years. Others, mainly breeders, were less sanguine, saying that transgenic trees could be available in the aforementioned timeframe, but that another 10 or more years would be required to verify stability and check for side effects before they could be planted and grown on a large scale. A few individuals argued that classical selection and breeding could turn one or possibly two generations in the same time and have clones with improved specific gravity, increased dry wood substance, and possibly lower lignin content in plantations, and perhaps in processing facilities.

Many specialists expressed concern about mechanisms for commercialization. Organizations engaged in transformation research patent their creations, and licensing fees or royalties are a worrisome issue. Large forest products companies have the wherewithal to license technology and transform their own breeding or production populations and/or fund joint ventures with molecular research organizations, but not all breeders and growers, including some larger, research-shy companies, have such latitude. This seems especially true for the numerous small enterprises most likely to grow biomass feedstock. To foster timely, efficient technology transfer, the BFDP may need to support independent laboratories that can license genetic constructs and effect transformation for cooperatives of small breeders, nurseries, and/or growers. Interviewees of all types and affiliations expressed concern about the "law of unintended consequences." To what extent can lignin be reduced? How will reducing lignin content affect growth, development, and resistance to stress/pests? As noted in a preceding section, caution seems necessary in that switchgrass lines bred for lower lignin content tend to also be less winter hardy (Vogel 12/07). Quick and easy answers, of course, are not available. Only time will tell. To answer these questions, future research should include well-designed, long-term field trials.

The biggest doubt expressed by practically all interviewees centered on acceptance of transgenic trees by a skeptical public. Even the most positive molecular geneticists were concerned that products of their long labors would not be used beyond research and education. A few argued, however, that trees genetically engineered to have low lignin content pose far less of a threat, if any, to the environment than those 
modified with genes for enhanced pest resistance. Regardless, most favored incorporation of sexual sterility to minimize risk and raise probability of public acceptance. Breeders, however, would prefer that they be able to switch sterility on and off so as to facilitate breeding as well as provide for safety in plantations. Favored also were responsible and transparent testing along with clear and consistent communication of risks, cost, and benefits. Support of research on these fronts seems imperative.

On the whole, interviewees considered modification of lignin/cellulose contents via genetic transformation a plus. Regardless of product, even minor improvement (lower lignin/higher cellulose) was viewed as having substantial financial benefit, provided the technology is accepted by the public. If naught else, transformation research has enlarged and will continue to enhance knowledge of cell wall biosynthesis, and improved understanding can only help all involved parties, present and future.

\section{SUMMARY AND CONCLUSIONS}

Genetic manipulation of enzymes involved in lignin biosynthesis has produced meaningful, if somewhat confusing, results. Substantial reductions in lignin content have been caused by suppression of 4CL, but such results have been the exception rather than the rule. Not to be overlooked, however, is the favorable impact of $4 \mathrm{CL}$ suppression on cellulose content and hemicellulose composition as well as lignin content.

Most interventions in lignin biosynthesis have provoked changes in composition. For example, suppression of COMT/OMT activities had little or no effect on quantity, while reducing SGR and increasing proportions of other constituents incorporated into lignin or lodged as free compounds in cell walls. To date,
CoAOMT manipulation has not provoked changes in either quantity or quality, but further research may be warranted. Interventions affecting CCR activity produced little or no impact on quantity, but yielded lignin with higher SGR, lower guaiacyl content, and less condensation. Some unusual compounds also resulted, and severe suppression caused abnormal growth. Suppression of CAD activity reduced quantity to some extent, while either not altering or slightly reducing SGR. The most frequent outcomes, however, were increased synthesis and incorporation of aldehydes in lignin as well as accumulation of atypical free compounds in cell walls.

Most such qualitative changes should ease alkaline pulping for papermaking, but views as to benefits for ethanol conversion generally are negative (Dimmel 09/23, Northey 10/06, Lewis 10/09). The value of higher SGR is questionable (Chiang, 12/07, Sewalt 12/07). More problematic, however, is increased quantities of aldehydes and phenolic groups in lignin as well as free compounds in cell walls (Chiang 12/07, Sewalt $12 / 07$ and 03/02). Such changes are likely detrimental to ethanol processing, since they could increase lignin degradation products in processing streams, complicate separation of lignin residues, and raise concentrations of undesirable chromophores in effluents. Changes in composition that are more desirable may include a more condensed structure, more acid-resistant composition, and fewer covalent linkages between lignin and hemicelluloses.

Modifications reported to date have not caused such changes, and much research on modifying composition remains to be done. In the meantime, attention should be focused on reducing lignin content, and the most feasible approach involves manipulation of 4CL activity. Increased emphasis on intervention in cellulose synthesis also is warranted.

Other important areas requiring additional research include: extent to which 
lignin/cellulose contents can be modified without adversely affecting growth, development, and stress/pest tolerance; identity and nature of rate limiting steps in all aspects of biosynthesis; nature and extent of genetic control in tissues and developmental stages; and enzymes/genes governing transport, storage, and polymerization.

On the development and technology transfer fronts, attention must be given to improving transformation efficiency, ensuring that transgenic trees can be used safely, moderating impacts of patents and licensing agreements, and founding/developing independent laboratories. Addressing these issues will ensure that useful and safe transgenic trees will be available to the variety of growers likely to become engaged in biomass production. Balanced support is necessary to ensure that dividends from this exciting and rapidly evolving technology are realized in a reasonable timeframe.

In sum, progress has been substantial. Lignin quantity can and has been reduced via genetic transformation. Changes in composition, though of questionable value, have also been achieved, and significant inroads have been made in terms of increasing cellulose content. Simultaneous intervention to reduce lignin and increase cellulose content could yield spectacular results. In consequence, the issue of feasibility has become one of when, rather than if transgenic trees will be available commercially, and 5 to 10 years, excluding additional time for testing of stability and safety, is a reasonable estimate.

\section{COPRODUCTS}

Much interest has been expressed in raising value of poplar feedstocks by modifying them to produce high-value coproducts as well as to raise efficiency of ethanol conversion. Thinking along these lines ranges from solid wood products through pulping chemicals and wood preservatives to enzymes and even pharmaceuticals. Past problems with xylose conversion prompted much research on manufacturing biodegradable plastics. Considerable effort has also been expended on developing uses for lignin, the least useful biomass constituent.

Coproducts could add to overall feedstock value, and trees conceivably can be modified to yield desirable materials. Vogel and other workshop participants (12/07) emphasized, however, that genetically modifying any plant to yield two or more products, e.g., ethanol and pharmaceuticals, would be difficult.
Individuals active in poplar modification confirmed this view, especially as concerns breeding. They nevertheless suggested that some traits favoring ethanol production, e.g., less lignin and more cellulose, are compatible with those needed for manufacturing value-added solid wood products. Accordingly, some poplar growers are considering changing breeding and cultural practices to produce largediameter logs from the lower 8-16 ft of stems for manufacture of molding and trim, veneer for reconstituted wood panels, and similar products. Remainder of the tree and manufacturing residues would be available for ethanol production. This seems the best approach to serious development of poplar coproducts for the near term.

The forest products industry is also investigating genetic transformation as a means to induce/enhance synthesis of pulping and other chemicals. Peter (10/18) 
and coworkers at the Institute of Paper Science and Technology have inserted a genetic construct stimulating anthroquinone synthesis in a model poplar clone. Outcomes are uncertain; practical utility requires synthesis levels 1000 -fold those in controls. An alternative is more modestly enhanced synthesis of this or other extractives compounds for use as natural wood preservatives.

Sterols derived from extractives are used to manufacture a so-called cholesterollowering margarine by Raisio Group, Finland, in collaboration with Johnson and Johnson, Inc. Similar products are marketed by Unilever N.V., The Netherlands. Such uses underscore the utility of trees as sources of valuable chemicals, but this particular market is well supplied by pulping by-products.

Given the fair quantity of alcohol, fatty acids, and waxes in poplar extractives, some potential exists for increasing synthesis and/or modifying chain lengths, changing double numbers and positions, or introducing desirable functional groups. Topfer et al. (1995) outlined advances in modifying oil crops to produce new oils and polymers, and argued that markets for nonfood products from plants could grow from an estimated $\$ 15$ million at time of their analysis to $\$ 320$ million by 2005 .

Modifications to synthesize pharmaceuticals seem best attempted in leafy, herbaceous crops, e.g., corn. Several firms, e.g., the Monsanto division of Pharmacia Corp. have been active on this front, but research by this organization has slowed in recent times. Production of human mucosal antibodies in corn by EPIcyte Pharmaceutical Corp., however, provides a successful example.

Lignin, currently, is most often used as fuel. The paper industry generates substantial proportions of process heat, steam, and electricity by combustion of lignin residues and simultaneously recovers most of its inorganic pulping chemicals.
Lignins, at least lignosulfonates, have some more valuable uses, e.g., animal feed and fertilizer binders, concrete modifiers, and adhesive extenders. Existing markers, however, are small; worldwide consumption of lignosulfonates totals only a million tons per year (Northey 10/06, Rasmussen 10/08). Research on lignin-based adhesives is aggressive, but they are not commercially practical at present. Lastly, markets for lignin coproducts would be swamped if the Organosolv or similar pulping processes are adopted on a large scale.

Several markets exist for xylose, e.g., animal feed and fertilizer binders, furfural, and an artificial sweetener, Xylitol. Only modest increases in demand, however, are expected over the near-term (Rasmussen 10/08, Jeffries 10/14). For the longer term, Cargill Dow Polymers Inc., a joint venture by Cargill Corp. and Dow Chemical Inc., plans to produce biodegradable polylactide plastic from xylose. A plant designed to produce 300 million lbs per year for textile and food packaging applications is slated for startup in 2002. Previous attempts to develop this product were limited by undesirable polymer properties and high costs. The firms claim to have far less expensive technology, and consider their product not only biodegradable but also performance and price competitive. Dow is also investing in alliances with biotechnology firms to acquire genes for producing industrial and agricultural chemicals.

In a related vein, Pharmacia Corp. has investigated transforming plants, e.g., corn, to synthesize polyhydroxyalkanoate, a biodegradable plastic, in vivo. The project has been shelved by the company but is being pursued by British and Swiss universities. Other organizations have used transformation to induce plant synthesis of yet other biodegradable polymers, e.g., polyhydroxybutyrate and polyhydroxyvalerate. 
Competition in polymers continues not only from the petrochemical industry but also from biological sources, i.e., bacterial synthesis. DuPont Co. is developing a genetically engineered bacterial system to produce a compound used to manufacture polyester. At least one competitor, Royal Dutch Petroleum Co., has developed an inexpensive chemical synthesis, and its products are expected to reach markets several years before the biobased version. Nonetheless, DuPont has committed to derive $25 \%$ of its annual revenues from renewable resources by 2010 . Transgenic crops figure heavily in its plans for producing polymers and specialty chemicals. Critics argue, however, that a generation, rather than 10 years, will pass before renewable resources replace a significant share of petrochemical products. Consensus estimates suggest that petroleum production will not peak until 2020, and that supplies will last for another 40-160 years. Fifty or more years could transpire before needs for plant-based products become critical.

Taking a different and potentially more lucrative tack, ethanol conversion could be improved by not only genetically modifying feedstock quality and quantity of trees but also by transforming them to synthesize processing enzymes. As noted in preceding sections, large enzyme quantities are needed and some feedstock constituents, especially lignin, inhibit enzymatic activity and hamper recycling. Against this background and high enzyme costs, transforming otherwise modified plants to produce cellulases and hemicellulases seems a lucrative exception to otherwise dim prospects for coproducts. As an example, Ziegler et al. (in press) transformed Arabidopsis thaliana with constructs designed to over-express synthesis of a thermostable endoglucanase. High levels of active enzyme accumulated in and could be extracted from intercellular spaces of leaf tissue. Other phenotypic characteristics appeared normal, an expected outcome since optimal temperatures for activity of the enzyme are well above normal growing temperatures. The authors indicated that other laboratories have obtained promising results with additional enzymes in different plants. Long-term potential of this approach warrants expansion to research on poplar wood and/or bark.

In sum, genetic modification of poplars to yield many high-value coproducts, e.g., pharmaceuticals, does not seem viable. Such efforts would entail trying to serve two or more markets with widely different requirements, and interfere with modifications to improve feedstock quality/quantity. No doubt, byproducts and residues, e.g., xylose, will be used, even absent genetic modification, to produce biobased polymers in regions where feedstock supplies are large and demand for such materials is high. On the positive side, modifications compatible with ethanol conversion seem lucrative, especially over the near-term. A prime example is changing breeding and cultural practices to produce value-added solid wood products. For the longer term, accelerated research on modifications to produce ethanol processing enzymes in trees seems warranted.

\section{WORKSHOP}

In accordance with the plan for preparation of this report, a workshop was organized and conducted in December 1999 by the author with assistance from Ms. L. L.
Wright of the Bioenergy Feedstock

Development Program.

Purposes and scope were: (1) Review and refine this report and a parallel one on 
switchgrass (Panicum virgatum); (2) Debate advantages and disadvantages of the several approaches to improving feedstock quality; and (3) Develop consensus positions on feasibilities of modification and recommendations for future research, development, and technology transfer. Findings and conclusions from the workshop were used to revise the aforementioned reports, recommend future program directions, and prepare a "technology roadmap" for use in program planning.

$$
\text { Participants and their affiliations }
$$
were:

Report Authors: Ronald J. Dinus, Consultant - Poplar Genetics \& Wood Quality; Ken P. Vogel, USDA/ARS Switchgrass Genetics.

Poplar Team: Vincent L. Chiang, MI Tech Univ. - Genetic Transformation; Peggy
Payne, Boise Cascade Corp. - Genetics \& Breeding; Mitchell M. Sewell, Consultant Genetic Markers.

Switchgrass Team: Robert V. Conger, Univ. of TN - Genetic Transformation; Hans G.

Jung, USDA/ARS - Cell Wall Biochemistry.

Independent Consultants: Clint C.S. Chapple, Purdue Univ. - Biochemistry; Vincent J.H. Sewalt, Pioneer Hybrid International Inc. - Genetic Transformation.

U. S. Department of Energy: Janet Cushman, Lynn L. Wright, and Jerry A. Tuskan - Bioenergy Feedstock Development Program, Oak Ridge National Laboratory; John Ferrell - Office of Fuels Development, Sarah Sprague - Office of Transportation Technologies; Steve Thomas - National Renewable Energy Laboratory; Gregory Dilworth - Energy Biosciences.

\section{ACKNOWLEDGMENTS}

The author expresses appreciation to the numerous individuals who gave freely of their time and thoughts during background research for this report. Much appreciated also is the kind help of Janet Cushman, Steve Thomas, Jerry Tuskan, and Lynn Wright with workshop organization and report preparation. Special thanks are given to Vincent Chiang, Peggy Payne, Vincent Sewalt, and Mitch Sewell for their thoughtful comments during the workshop and revision of the report, and to Linda Belles Dinus and Polly Henry for assistance with report preparation and editing.

\section{PERSONAL COMMUNICATIONS}

Beatson, Roger - Univ. of British Columbia, Dept. of Wood Science, 08/02/99

Bradshaw, Toby - University of Washington, 09/17/99

Chiang, Vincent - Michigan Technological University, 09/15 and 12/07/99

Dean, Jeff - University of Georgia, 09/24/99

Dimmell, Don - Institute of Paper Science and Technology, 09/23/99 
Douglas, Carl - Univ. of British Columbia, Dept. of Botany, 10/13/99

Ellis, Dave and Ben Sutton - BC Research, 09/30/99

Glassner, David - National Renewable Energy Laboratory, 09/01/99

Hettenhaus, Jim - Chief Executive Assistance, Charlotte, NC, 09/01/99

Jeffries, Tom - USDA For. Serv., Forest Products Laboratory, 10/14/99

Keeling, Peter - ExSeed Genetics, 09/03/99

Lewis, Norm - Washington State University, 10/09/99

Loopstra, Carol - Texas A \& M, 10/19/99

Merkle, Scott - University of Georgia, 09/23/99

Northey, Bob - University of Washington, 10/06/99

Peter, Gary - Institute of Paper Science and Technology, 10/18/99

Pilate, Gilles - Unite Amelioration, Genetique, Physiologie Forestieres, 10/18/99

Purnell, Bob - International Paper Co., 10/18/99

Rasmussen, Glenn - Ligno-Tech USA, Inc., 10/08/99

Rousseau, Randy - Westvaco Corp., 09/29/99

Ruth, Mark - National Renewable Energy Laboratory, 08/18/99

Savidge, Rod - University of New Brunswick, 09/23/99

Sederoff, Ron - North Carolina State Univ., 09/29/99

Sewelt, Vincent - Pioneer Hybrid International, Inc., 12/07 and 03/02/99

Strauss, Steve - Oregon State University, 10/01/99

Thomas, Steven - National Renewable Energy Laboratory, 10/08/99

Tuskan, Jerry - Bioenergy Feedstock Development Program, 08/01 and 01/07/99

Vogel, Ken - USDA/ARS, University of Nebraska, 12/07/99

\section{LITERATURE CITED}

Alstrom-Rapaport, C., Lascoux, M., Wang Y. C., and Tuskan, G. A. 1998. Identification of a RAPD marker linked to sex determination in the basket willow (Salix viminalis L.). $J$. Hered. 89:44-49.

Arioli, T., Peng L.C., Betzner A. S., Burn, J., Wittke W., Herth W., Camilleri C., Hofte H., Plazinski J., Birch R., Cork A., Glover J., Redmond J., and Williamson, R.E. 1998. Molecular analysis of cellulose biosynthesis in Arabidopsis. Science. 279:717-20.

Bao, W., O'Malley, D. M., Whetten, R. and Sederoff, R. R. 1993. A laccase associated with lignification in loblolly pine xylem. Science: 260:672-674.

Bate, N. J., Orr, J., Ni, W., Meromi, A., Nadler-Hasa, T., Doerner, P. W., Dixon, R. A., Lam, C. J. and Elkind, Y. 1994. Quantitative relationship between phenylalanine ammonia-lyase levels and phenylpropanoid accumulation in transgenic tobacco identifies a ratedetermining step in natural product synthesis. Proc. Natl. Acad. Sci. USA. 91(15-16): 7608-7612.

Baucher, M., Chabbert, B., Pilate, G., vanDoorsselaere, J., Tollier M.-T., Petit-Conil M., Cornu, D., Monties, B., vanMontagu, M., Inze, D., Jouanin, L., and Boerjan, W. 1996. Red xylem and higher lignin extractability by down-regulating a cinnamyl alcohol dehydrogenase in poplar. Plant Physiol. 112:1479-1490.

Baucher, M., Christensen, J. H., vanDoorsselaere, J., Meyermans, H., Chen, C., Burggraeve, B., Leple J-C., Pilate, G., Petit-Conil, M., Jouanin, L., Chabbert, B., Monties, B., vanMontagu, M., and Boerjan, W. 1997. Molecular tools to study lignin biosynthesis in poplar. 
Mededelingen Faculteit Landbouwkundige en Toegepaste Biologische Wetenschappen Universiteit Gent. 62:(4AB):103-1411.

Beavis, W. D., Grant, D., Albertsen, M., and Fincher, R. 1991. Quantitative trait loci for plant height in four maize populations and their associations with qualitative genetic loci. Theor. Appl. Genet. 83:141-145.

Bertolucci, F. L. G., Demuner, B. J., Garcia, S. L. R., and Ikemori, Y. K. 1995. Increasing fiber yield and quality at Aracruz. In: Eucalpyt plantations: Improving fibre yield and quality. Proc. CRC-IUFRO Conf., 19-24 Feb. pp. 33-34. Potts, B. M., Borralho, N. M. G., Reid, J. B., Cromer, R. N., Tibbits, W. N., and Raymond, C. A., Eds. CRC for Temperate Hardwood Forestry. Hobart, Australia.

Boerjan, W., Meyermans, H., Chen, C., Christensen, J., Leple, J.-C., Pilate, G., Lapierre, C., Pollet, B., Jouanin, L., Baucher, M., vanDoorsselaere, J., Petit-Conil, M., and vanMontagu, M. 1999. Improved wood quality for the pulp and paper industry by genetic engineering of lignin biosynthesis. Abstr. In: Program International Poplar Symposium II. p. 18. Sept 13-17, 1999. Orleans, FR.

Borralho, N. M. G., Cotterill, P. P, and Kanowski, P. J. 1993. Breeding objectives for pulp production of Eucalyptus globulus under different industrial cost structures. Can. J. For. Res. 23:648-656.

Boudet, A. M., Lapierre, C., and Grima-Pettenati, J. 1995. Biochemistry and molecular biology of lignification. New Phytol. 129:203-236.

Boudet, A. M. and Grima-Pettenati, J. 1996. Lignin genetic engineering. Mol. Breeding. 2(1):25-39.

Bradshaw, J. D., Jr. Molecular genetics of Populus. 1996. In: Biology of Populus and Its Implications for Management and Conservation. pp. 183-199. Stettler, R. F., Bradshaw, H. D. Jr., Heilman P. E., and Hinckley, T. M., Eds. NRC-CNRC Research Press, Ottawa, Canada.

Bradshaw, J. D., Jr. and Foster, G. S. 1992. Marker-aided selection and propagation systems in trees: Advantages of cloning for studying quantitative inheritance. Can. J. For. Res. 22(7):1044-1049.

Bradshaw, J .D., Jr. and Stettler, R. F. 1995. Molecular genetics of growth and development in Populus. IV. Mapping QTLs with large effects on growth, form and phenology traits in a forest tree. Genetics. 139:963-973.

Bradshaw, J. D., Jr., Villar, M., Watson, N. D., Otto, K. G., Stewart, S., and Stettler, R. F. 1994. Molecular genetics of growth and development in Populus. III. A genetic linkage map of a hybrid poplar composed of RFLP, STS, and RAPD markers. Theor. Appl. Genet. 89:67-178.

Brondani, R. P. V., Brondani, C., Tarchini, R., and Grattapaglia, D. 1998. Development and mapping of microsatellite based markers in Eucalyptus. Theor. Appl. Genet. 97:816-827.

Bridgwater, F. E., Talbert, J. T., and Jahromi, S. 1983. Index selection for increased dry weight in a young loblolly pine populations. Silvae Genetica. 32:5-6.

Campbell, M. M. and Sederoff, R. R. 1996. Variation in lignin content and composition: Mechanism of control and implications for the genetic improvement of plants. Plant Physiol. 110:3-13.

Campinhos, I. N., Grattapaglia, D., Bertolucci, F. L., and Alfenas, A. 1996. Stability of expression of QTL alleles controlling growth across variable genetic backgrounds in Eucalyptus grandis. In: Proc. International IUFRO on Eucalyptus Genetics and Silviculture. Vol. 2. pp. 186-191. Salvador, Brazil. 
Cervera, M. T., Gusmao, J., Steenackers, M., Peleman, J., Storm., V., VanderBroeck, A., Van Montagu, M., and Boerjan, W. 1996. Identification of AFLP molecular markers for resistance against Melampsora larici-populina in Populus. Theor. Appl. Genet. 93:733-737.

Cervera, M. T., Villar, M., Faivre-Rampant, P., Goue, M.-C., vanMontagu, M., and Boerjan, W. 1997. Application of molecular marker technologies in Populus breeding. In:

Micropropagation, Genetic Engineering and Molecular Biology of Populus. pp. 101-115. Klopfenstein, N .B., Chun, Y. W., Kim, M.-S., and Ahuja, M. R., Eds. USDA. Forest Service Gen.Tech.Rep. RM-GTR-297.

Chandler, S.F. 1995. Commercialisation of genetically engineered trees. In: Eucalypt plantations: Improving fibre yield and quality. Proc. CAC-IUFRO Conf., 19-24 Feb. pp. 381-385. Potts, B. M., Borralho, N. M .G., Reid, J. B., Cromer, R. N., Tibbits, W. N., and Raymond, C. A., Eds. CAC for Temperate Hardwood Forestry. Hobart, Australia.

Clarke, C. R. E. and Wessels, A. E. 1995. Variation and measurement of pulp properties in Eucalypts. In: Eucalypt plantations: Improving fibre yield and quality. Proc. CAC-IUFRO Conf., 19-24 Feb. pp. 93-100. Potts, B. M., Borralho, N. M. G., Reid, J. B., Cromer, R. N., Tibbits, W. N., and Raymond, C. A., Eds. CAC for Temperate Hardwood Forestry. Hobart, Australia.

Costa E Silva, J., Wellendorf, H., and Pereira, H. 1998. Clonal variation in wood quality and growth in young Sitka spruce (Picea sitchensis Bong.) Carr.): Estimation of quantitative genetic parameters and index selection for improved pulpwood. Silvae Genetica. 47(1):20-33.

Crocker, T. P. 1995. Middle range infrared diffuse reflectance method for measuring lignin content of Pinus taeda increment cores. Independent Student Research Project Report, Institute of Paper Science and Technology, Atlanta, GA.

Davis, M., Megraw, R., Sewell, M., Evans., Neale, D., West, D., Elam, C., Wislogel, A., Wheeler, N., Jech, K., Tuskan, G., and Dinus, R. 1999. Application of pyrolysis molecular beam mass spectrometry for the determination of loblolly pine and hybrid poplar cell wall composition. In: Proc. 1999 TAPPI Pulping Conference. 3:1077-1081. TAPPI Press, Atlanta, GA.

Dean, J. and Ericksson, K. E. Biotechnological modification of lignin structure and composition in forest trees. 1992. Holzforschung. 46:135-147.

Delmer, D. P. and Amor, Y. 1995. Cellulose biosynthesis. Plant Cell. 7:987-1000.

Delmer, D. P. 1999. Cellulose biosynthesis: Exciting times for a difficult field of study. Annu. Rev. Plant Physiol. Plant Mol. Biol. 50:245-76.

Dinus, R. J. 1999. Unpublished data. Wood chemistry analyses of hybrid poplar and loblolly pine standards. US Dept. of Energy, ORNL-ESD-BFDP, Consulting Contract No. 54XTA060V.

Dinus, R. J. and Tuskan, G. A. 1997. Integration of molecular and classical genetics: A synergistic approach to tree improvement. In: Micropropagation, Genetic Engineering and Molecular Biology of Populus. pp. 220-235. Klopfenstein, N. B., Chun, Y. W., Kim, M.-S., and Ahuja, M. R., Eds. USDA. Forest Service Gen.Tech.Rep. RM-GTR-297.

Dinus, R. J. and Welt, T. 1997. Tailoring fiber properties to paper manufacture: Recent developments. TAPPI J. 80(4):127-139.

Dinus, R. J., Dimmel, D. R., Feirer, R. P., Johnson, M. A., and Malcolm, E. W. 1990. Modifying woody plants for efficient conversion to liquid and gaseous fuels. US Dept. of Energy, Oak Ridge National Laboratory, Environmental Sciences Division, Biomass Production Program Report No. ORNL/Sub/88-SC006/1. 
Donaldson, L. A. 1995. Cell wall fracture properties in relation to lignin distribution and cell differentiation among three genetic groups of radiata pine. Wood Sci. and Tech. 29(1):51-63.

Donaldson, L. A., Croucher, M., and Uprichard, J. M. 1997. Clonal variation of wood chemistry variables in radiata pine (Pinus radiata (D. Don)) wood. Holzsforschung. 51:537-542.

Doorsselaere, J. van, Baucher, M., Chognot, E., Chabbert, B., Tollier, M. T., Petit-Conil, M., Leple, J. C., Pilate, G., Cornu, D., and Monties, B. 1995. A novel lignin in poplar trees with a reduced caffeic acid/5-hydroxyferulic acid O-methyltransferase activity. Plant J. 8(6):855-864.

Douglas, C. J., Cukovic, D., Pereira, J., and Allina, S. 1999. The Populus 4-coumarate:CoA ligase (4CL) gene family and antisense suppression of 4CL activity in transgenic Populus clone. INRA 717-1B4. Abstr. In: Program International Poplar Symposium II. p. 28. Sept 13-17, 1999. Orleans, FR.

Dwivedi, U. N., Campbell, W .H., Yu, J., Datla, R. S. S. Bugos, R. C., Chaing, V. L., and Podila, G. K. 1994. Modification of lignin biosynthesis in transgenic Nicotiana through expression of an antisense O-methyltransferase gene from Populus. Plant Mol. Biol. 26(1):61-71.

Evans, R. and Downes, G. 1995. Recent developments in automated wood quality assessment. In: Eucalypt plantations: Improving fibre yield and quality. Proc. CRC-IUFRO Conf., 19-24 Feb. pp. 93-100. Potts, B. M., Borralho, N. M. G., Reid, J.B., Cromer, R. N., Tibbits, W. N., and Raymond, C. A., Eds. CRC for Temperate Hardwood Forestry. Hobart, Australia.

Ferreira, M. and Santos, P. E. T. 1995. Eucalypts wood traits for species/provenances/plus trees and clones planted in Brazil--A review applied to genetic improvement for pulp production. In: Eucalypt plantations: Improving fibre yield and quality. Proc. CRCIUFRO Conf., 19-24 Feb. pp. 258-260. Potts, B. M., Borralho, N. M. G., Reid, J. B., Cromer, R. N., Tibbits, W. N., and Raymond, C. A., Eds. CRC for Temperate Hardwood Forestry. Hobart, Australia.

Fillatti, J. J., Sellmer, J., McCown, B., Haissig, B., and Comai, L. 1987. Agrobacterium mediated transformation and regeneration of Populus. Mol. Gen. Genet., 106:192-199.

Fukushima, K., Chen, F., and Yasuda, S. 1999. A novel biosynthetic pathway that regulates the ratio of syringyl to guaiacyl residues in lignin. Abstr. In: Program International Poplar Symposium II. p. 35. Sept 13-17, 1999. Orleans, FR.

Goldstein, I. S. and Easter, J. M. 1992. An improved process for converting cellulose to ethanol. TAPPI J. 75(8):135-140.

Goue-Mourier, M. C., Faivre-Rampant, P., LeGuerroue, B., Lefevre, F. and Villar, M. Molecular and genetic approaches to rust resistance (Melampsora sp.) in poplar (Populus sp.). 1996. In: Somatic Cell Genetics and Molecular Genetics of Trees. pp. 249-254. Kluwer Academic Publishers. The Netherlands.

Grado, S. C. and Chandra, M. J. 1998. A factorial design analysis of a biomass to ethanol production system. Biomass and Bioenergy. 15(2):115-124.

Grattapaglia, D. (In press) Molecular breeding of Eucalyptus: State of the art, applications and technical challenges. In: Molecular Markers and Genome mapping in Woody Plants. Jain, S. M. Ed. Kluwer Academic Publishing. The Netherlands.

Grattapaglia, D., Bertolucci, F. L., and Sederoff, R. 1995. Genetic mapping of QTL's controlling vegetative propagation in Eucalyptus grandis and Eucalyptus urophylla using a pseudotestcross strategy and RAPD markers. Theor. Appl. Genet. 90:933-947. 
Greaves, B. L., Borralho, N. M. G., Raymond, C. A., and Farrington, A. 1996. Use of Pilodyn for the indirect selection of basic density in Eucaluptus nitens. Can. J. For. Res. 26:1643-1650.

Gregg, D. and Saddler, J. N. 1995. Bioconversion of lignocellulose residue to ethanol: Process flowsheet development. Biomass and Bioenergy. 9(1-5):287-302.

Gregg, D. and Saddler, J. N. 1996. A techno-economic assessment of the pretreatment and fractionation steps of a biomass-to-ethanol process. Appl. Biochem. Biotech. 57/58:711-726.

Gregg, D., Boussaid, A., and Saddler, J. N. 1998. Techno-economic evaluations of a generic wood-to-ethanol process: Effect of increased cellulose yields and enzyme recycle. Bioresource Tech. 63:7-12.

Grima-Pettenati, J., Goffner, D., Teulieres, C., and Boudet, A. M. 1996. Lignification genes in Eucayptus: Characterization, expression and subsequent use for lignin manipulation. In: Proceedings somatic cell genetics and molecular genetics of trees. September 26-30, 1995, pp. 137-145. Ahuja, M. R., Boerjan,W., and Neale, D. B., Eds. Gent, Belgium.

Grover, A. T., Devey, M. E., Fiddler, T. A., Lee, J. M., Megraw, R. A. , Mitchell-Olds, T., Sherman, B. K., Vujcic, S. L., Williams, C. G., and Neale, D. B. 1994. Identification of quantitative trait loci influencing wood specific gravity in an outbred pedigree of loblolly pine. Genetics. 138:1293-1300.

Halpin, C., Knight, M. E., Foxon, G. A., Campbell, M. M., Boudet, A. M., Boon, J. J., Chabbert, B., Tollier, M-T., and Schuch, W. 1994. Manipulation of lignin quality by downregulaton of cinnamyl alcohol dehydrogenase. Plant J. 6(3): 339-350.

Han, K.-H., Gordon, M. P., and Strauss, S. H. 1996. Cellular and molecular biology of Agrobacterium-mediated transformation of plants and its application to genetic transformation of Populus. In: Biology of Populus and Its Implications for Management and Conservation. pp. 201-222. Stettler, R. F., Bradshaw, H. D. Jr., Heilman P. E., and Hinckley, T. M., Eds. NRC-CNRC Research Press, Ottawa, Canada.

Harcourt, R., Kyozuka, J., Zhu, X., Southerton, S. Llewellyn, D., Dennis, E., and Peacock, J. 1995. Genetic engineering for sterility in temperate plantation Eucalypts. In: Eucalypt plantations: Improving fibre yield and quality. Proc. CRC-IUFRO Conf., 19-24 Feb. pp. 403-405. Potts, B. M., Borralho, N. M. G., Reid, J. B., Cromer, R. N., Tibbits, W. N., and Raymond, C. A., Eds. CRC for Temperate Hardwood Forestry. Hobart, Australia.

Herpka, I. 1990. Importance of wood basic density for maximization of total yield in short rotation poplar biomass production. In: Improvement of feedstock quality. Proc. Second IEA/BA Task V Workshop. pp. 49-85. Toronto, Canada.

Hu, W.-J., Akiyoshi, K., Tsai, C.-J., Lung, J., Osakabe, K., Ebinuma, H., and Chiang, V. L. 1998. Compartmentalized expression of two structurally and functionally distinct 4coumarate:CoA ligase genes in aspen (Populus tremuloides). Proc. Natl. Acad. Sci. USA. 9:5407-5412.

Hu, W.-J., Harding, S.A., Lung, J., Popko, J. L., Ralph, J., Stokke, D. D., Tsai, C. J., and Chiang, V. L. 1999. Repression of lignin biosynthesis promotes cellulose accumulation and growth in transgenic trees. Nature Biotech. 17:808-812.

Jett, J. B., Weir, R. J., and Barker, J. A. 1977. The inheritance of cellulose in loblolly pine. TAPPI For. Biol. Comm. Meet. Madison, WI, 4pp. TAPPI Press, Atlanta, GA.

Johnson, G. R., Wheeler, N. C., and Strauss, S. H. 1999. (unpublished) Economic feasibility of marker aided selection in Douglas fir. U.S.D.A. For. Ser., Corvallis, OR.

Knott, S. A., Neale, D. B., Sewell, M. M., and Haley, C. S. 1997. Multiple marker mapping of quantitative trait loci in an outbred pedigree of loblolly pine. Theor. Appl. Genet. 94: 810-820. 
LaCombe, E., Hawkins, S., van Doorselaere, J., Piquemal, J., Goffener, D., Poeyslomenge, O., Boudet, A.-M., and Grima-Pettenati, J. 1997. Cinnamoyl CoA reductase, the first committed enzyme of the lignin branch biosynthetic pathway: Cloning, expression, and phytogenetic relationships. Plant J. 11(3):429-441.

Lapierre, C., Pollet, B., Petit-Conil, M., Toval, G., Romero, J., Pilate, G., Leple J.-C., Boergan, W., Ferret, V., DeNadai, V., and Jouanin, L. 1999. Structural alternations of lignins in transgenic poplars with depressed cinnamyl alcohol dehydrogenase of caffeic acid Omethyltansferase activity have an opposite impact on the efficacy of industrial kraft pulping. Plant Physiol. 119(1):153-163.

Li, L., Osakabe, Y., Joshi, C. P., and Chaing, V. L. 1999. Secondary xylem-specific expression of caffeoyl-coenzyme A 3-O methyltransferase plays an important role in the methylation pathway associated with lignin biosynthesis in loblolly pine. Plant Mol. Biol. 40:555-565.

Li, L., Popko, J. L., Umezawa, T., and Chiang, V. L. 2000. 5-hydroxyconiferyl aldehyde modulates enzymatic methylation for syringyl monolignol formation, a new view of monolignol biosynthesis in angiosperms. J. Biol. Chem. 275(9):6537-6545.

Loopstra, C., Eun-Gyu, N., Zho, Y., Puryear, J., Hongyan, W., and Pawlak, D. 1998. Regulation and function of xylem-specific genes in forest trees. Abstr. In: 1998 Information Exchange Group-40 Workshop: Wood and wood fibers: Properties and genetic improvement. Institute of Paper Science and Technology, July 19-22, 1998. Atlanta, GA.

Lowe, W. J. and Byram, T. W. 1995. Heritability estimates for loblolly pine wood specific gravity based on control-pollinated genetic tests. In: Proc. 23rd Southern Forest Tree Improvement Conf., pp. 204-209. Asheville, N.C.

Lowe, W. J., Byram, T. D., and Bridgwater, F. E. 1999. Selecting loblolly pine parents for seed orchards to minimize the cost of producing pulp. For. Sci. 45(2):213-216.

MacKay, J. J., O’Malley, D. M., Presnell, T., Booder, F. L., Campbell, M. M., Whetten, R. M., and Sederoff, R. R. 1997. Inheritance, gene expression, and lignin characterization in a mutant pine deficient in cinnamyl alcohol dehydrogenase. Proc. Natl. Acad. Sci. USA. 94(15):8255-8260.

Malan, F. S. and Arbuthnot, A. L. 1995. The inter-relationships between density and fibre properties of South African grown Eucalyptus grandis. In: Eucalypt plantations: Improving fibre yield and quality. Proc. CRC-IUFRO Conf., 19-24 Feb. pp. 116-120. Potts, B. M., Borralho, N. M. G., Reid, J. B., Cromer, R. N., Tibbits, W. N., and Raymond, C. A., Eds. CRC for Temperate Hardwood Forestry. Hobart, Australia.

Marita, J. M., Ralph, J., Hatfield, R. D., and Chapple, C. 1999. NMR characterization of lignins in Arabidopsis altered in the activity of ferulate 5-hydroxylase. Proc. Natl. Acad. Sci. USA. 96(22): $12328-12332$.

McDougall, G .J. 1998. Purification of coniferyl alcohol oxidase from lignifying xylem of Sitka spruce using immobilised metal affinity chromatography. J. Plant Physiol. 153:539-544.

McLaughlin, S. B., Samson, R., Bransby, D., and Wiselogel, A. 1996. Evaluating physical, chemical, and energetic properties of perennial grasses as biofuels. In: Bioenergy '96 Seventh National Bioenergy Conf.: Partnerships to develop and apply biomass technologies, Sept. 15-20, 1996. pp. 1-8. Nashville, TN.

Meilan, R. and Strauss, S. H. 1997. Poplar genetically engineered for reproductive sterility and accelerated flowering. In: Micropropagation, Genetic Engineering and Molecular Biology of Populus. pp. 212-219. Klopfenstein, N. B., Chun, Y. W., Kim, M.-S., and Ahuja, M. R., Eds. USDA. Forest Service Gen.Tech.Rep. RM-GTR-297.

Michell, A .J. and Schimleck, L. R.1995. Infrared spectroscopy - A rapid means of assessing pulping qualities of Eucalypt woods? In: Eucalypt plantations: Improving fibre yield and 
quality. Proc. CRC-IUFRO Conf., 19-24 Feb. pp. 121-123. Potts, B. M., Borralho, N. M. G., Reid, J. B., Cromer, R. N., Tibbits, W. N., and Raymond, C. A., Eds. CRC for Temperate Hardwood Forestry. Hobart, Australia.

Newcombe, G., Bradshaw, H. D., Castagner, G. A., and Stettler, R. F. 1996. A major gene for resistance to Melampsora medusae f.sp deltoidae in a hybrid poplar pedigree.

Phytopathology. 86:87-94.

Osakabe, K., Tsao, C. C., Li, L., Popko, J. S., Umezawa, T., Carraway, D. T., Smeltzer, R. H., Joshi, C. P., and Chiang, V. L. 1999. Coniferyl aldehyde 5-hydroxylation and methylation direct syringyl lignin biosynthesis in angiosperms. Proc. Natl. Acad. Sci. USA 96:8955-8960.

Olson, J. R., Jourdain, C. R., and Rousseau, R. J. 1985. Selection for cellulose content, specific gravity and volume in young Populus deltoides clones. Can. Jour. For. Res. 15:393-396.

Pilate, G., Leple, J. C., Noel., N., deNadai, V., Jouanin, L., Pollet, B., Mila., I., Vallet., C., and Lapierre, C. 1999. Abstr. In: Program International Poplar Symposium II. Sept 13-17, 1999. p. 71. Orleans, FR.

Piquemal, J., Lapiere, C., Myton, K., O’Connell, A., Schuch, W., Grima-Pettenati, J., and Boudet, A.-M. 1998. Down-regulation of cinnamoyl-CoA reductase induces significant changes of lignin profiles in transgenic tobacco plants. Plant Jour. 113(1):71-83.

Potts, B. M., Borralho, N. M. G., Reid, J. B., Cromer, R. N., Tibbits, W. N., and Raymond, C. A. (eds). 1995. Eucalypt plantations: Improving fibre yield and quality. Proc. CRC-IUFRO Conf., 19-24 Feb. CRC for Temperate Hardwood Forestry. Hobart, Australia.

Ralph, J., MacKay, J., Hatfield, R., O’Malley, D. M., Whetten, R. W., and Sederoff, R. R. 1977. Abnormal lignin in a loblolly pine mutant. Science. 277:235-239.

Raymond, C. A. Genetic control of wood and fibre traits in Eucalypts. 1995. In: Eucalypt plantations: Improving fibre yield and quality. Proc. CRC-IUFRO Conf., 19-24 Feb. pp. 49-52. Potts, B. M., Borralho, N. M. G., Reid, J. B., Cromer, R. N., Tibbits, W. N., and Raymond, C. A., (eds.). CRC for Temperate Hardwood Forestry. Hobart, Australia.

Rossitza, A., Favel, N., Martz, F., Chabbot, B., Tollier, M.-T., Montier, B., Fritig, B., and Legrand, M. 1995. Altered lignin composition in transgenic tobacco expressing Omethyltransferase sequences in sense and antisense orientation. Plant Jour. 8(4):465-477.

Savidge, R. A. 1986. Prospects for genetic manipulation of wood quality. In: New ways in forest genetics, Part 2 Proc. 20th meeting Canadian tree improvement association. Quebec City, Quebec, Canada, Aug. 19-22, 1985, pp. 159-165. Caron, F., Corriveau, A. G., and Boyle, F. J. B. Eds. Canadian Forestry Service, Ottawa, Canada.

Savidge, R. A. and Udagama-Randeniya, P. 1992. Cell wall-bound coniferyl alcohol oxidase associated with lignification in conifers. Phytochemistry. 31:2959-2966.

Savidge, R. A. and Forster, H. 1998. Seasonal activity of uridine 5'-diphosphoglucose: coniferyl alcohol glucosyltransferase in relation to cambial growth and dormancy in conifers. Can. $J$. Bot. 76:486-493.

Savidge, R. A., Udagama-Randeniya, P., Xu, Y., Leinhos, V., and Forster, H. 1998. In: Proc. Am Chem. Soc. Symposium, Series 697, Lignin and lignan biosynthesis. pp. 109-130. Lewis, N. G. and Sarkanen, S., Eds. Am. Chem. Soc.

Schimleck, L.R. and Michell, A. J. 1998. Determination of within-tree variation of kraft pulp yield using near-infrared spectroscopy. TAPPI J. 81(5):229-236.

Sewalt, V. J. H., Glasser, W. G., and Beauchemin, K .A.. 1997a. Lignin impact of fiber degradation. 3. Reversal of inhibition of enzymatic hydrolysis by chemical modification of lignin and by additives. J. Agric. Food Chem. 45(5):1823-1828. 
Sewalt, V. J. H., Ni, W., Jung, H. G., and Dixon, R. A. 1997b. Lignin impact on fiber degradation: Increased enzymatic digestibility of genetically engineered tobacco (Nicotiana tabacum) stems reduced in lignin content. J. Agric. Food Chem. 45(5): 1977-1983.

Sewell, M. M. and Neale, D. B. (In press) Mapping quantitative traits in forest trees. In: Molecular Biology of Woody Plants. Jain, S. M and Minocha, S. C., Eds. Kluwer Academic Publishers. The Netherlands.

Sewell, M. M., Bassoni, D. L., Megraw, R. A., Wheeler, N. C., and Neale, D. B. (In press) Identification of QTLs influencing wood property traits in loblolly pine (Pinus taeda L.). I. Physical wood properties: wood specific gravity, volume percentage of latewood and microfibril angle. Theor. Appl. Genet.

Sewell, M., Bassoni, D., Neale, D., Megraw, R., Elam, C., Davis, M., Wheeler, N., and Tuskan, G. Mapping quantitative traits for wood properties in loblolly pine. Abstr. In: 1998 Information Exchange Group-40 Workshop: Wood and wood fibers: Properties and genetic improvement. Institute of Paper Science and Technology, July 19-22, 1998. Atlanta, GA.

Sterjiades, R., Dean, J. F. D., and Eriksson, K.-E. L. 1992. Laccase from sycamore maple (Acer pseudoplatanus) polymerizes monolignols. Plnt. Physiol. 99:1162-1168.

Sterjiades, R., Dean, J. F. D., Gamble, G., Himmelsback, D. S., and Eriksson, K.-E. L. 1993. Extracellular laccases and peroxidases from sycamore maple (Acer pseudoplatanus) cellsuspension cultures. Reactions with monolignols and lignin model compounds. Planta. 190:75-87.

Sterky, F., Regan, S., Karlsson, J., Hertzberg, M., Rohde, A., Holmberg, A., Amini, B., Bhalerao, R., Larsson, M., Villarroel, R., vanMontagu, M., Sandberg, G., Olsson, O., Teeri, T. T., Boerjan, W., Gustafsson, P., Uhlen, M., Sundberg, B., and Lundberg, J. 1998. Gene discovery in the wood-forming tissues of poplar: analysis of 5692 expressed sequence tags. Proc. Natl. Acad. Sci. USA. 95(22):13330-13335.

Stettler, R. F., Zsuffa, L., and Wu, R. The role of hybridization in the genetic manipulation of Populus. 1996. In: Biology of Populus and Its Implications for Management and Conservation. pp. 87-112. Stettler, R. F., Bradshaw, H. D. Jr., Heilman P. E., and Hinckley, T. M., Eds. NRC-CNRC Research Press, Ottawa, Canada.

Stewart, D., Yahiaoui, N., McDougall, G., Myton, G., Marque, C., Boudet, A., and Haigh, J. 1997. Fourier-transform infrared and Raman spectroscopic evidence for the incorporation of cinnamaldehydes into the lignin of transgenic tobacco (Nicotiana tabacum L.) plants with reduced expression of cinnamyl alcohol dehydrogenase. Planta. 201:311-318.

Strauss, S. H., Lande, R., and Namkoong, G. Limitations of molecular-marker aided selection in forest tree breeding. 1992. Can J. For. Res. 22(7):1050-1061.

Teasdale, R.D. Optimising the application of biotechnology to forest plantations. In: Eucalypt plantations: Improving fibre yield and quality. Proc. CRC-IUFRO Conf., 19-24 Feb. pp. 427-430. Potts, B. M., Borralho, N. M. G., Reid, J. B., Cromer, R. N., Tibbits, W. N., and Raymond, C. A., Eds. CRC for Temperate Hardwood Forestry. Hobart, Australia.

Tibbits, W. and Hodge, G. 1998. Genetic parameters and breeding value predictions for Eucalyptus nitens. Wood fiber production traits. For. Sci. 44(4):587-598.

Timmis, R. and Trotter, P. C. 1989. Biological and economic feasibility of genetically engineered trees for lignin properties and carbon allocation. In: Proc. International workshop on applications of biotechnology in forestry and horticulture. pp. 349-367. Plenum Press, NY.

Topfer, R., Martini, N., and Schell, J. 1995. Modification of plant lipid synthesis. Science. 268(5211):681-686. 
Tsai, C.-J., Popko, J. L., Mielke, M. R., Hu, W.-J., Podila, G. K. and Chiang, V. L. 1998. Suppression of O-methyltransferase gene by homologous sense transgene in quaking aspen causes red-brown wood phenotypes. Plant Physiol. 117:101-112.

Tuskan, G.A. 1998. Short-rotation woody crop supply systems in the United States: What do we know and what do we need to know? Biomass and Bioenergy. 14(4):307-315.

Tuskan, G. A., West, D. C., Davis, M., and Bradshaw, H. D. 1999b. Variation in cellwall properties in Populus. Abstr. In: Proc. International Poplar Symposium II. Sept 13-17, 1999. p. 93. Orleans, FR.

Tuskan, G. A., Francis, K. E., Russ, S. L., Romme, W. H., and Turner, M. G. 1996. RAPD markers reveal diversity within and among clonal and seedling stands of aspen in Yellowstone National Park, U. S. A. Can. J. For. Res. 26:2088-2098.

Tuskan, G., West, D., Bradshaw, H. D., Neale, D., Sewell, M., Wheeler, N., Megraw, B., Jech, K., Wiselogel, A., Evans, R., Elam, C., Davis, M., and Dinus, R. 1999a. Two highthroughput techniques for determining wood properties as part of a molecular genetics analysis of hybrid poplar and loblolly pine. Appl. Biochem. Biotech. 77-79:55-65.

Udagama-Randeniya, P. V. and Savidge, R. A. 1995. Coniferyl alcohol oxidase - a catechol oxidase. Trees. 10:102-107.

Vaillancourt, R. E., Potts., B. M., Watson, M., Volker, P. W., Hodge, G. R., Reid, J. B., and West, A. K. Prediction of heterosis using RAPD generated genetic distances between Eucalyptus globulus trees. 1995. In: Eucalypt plantations: Improving fibre yield and quality. Proc. CRC-IUFRO Conf., 19-24 Feb. pp. 455-456. Potts, B. M., Borralho, N. M. G., Reid, J. B., Cromer, R. N., Tibbits, W. N., and Raymond, C. A., Eds. CRC for Temperate Hardwood Forestry, Hobart, Australia.

van Buijtenen, J. P. 1997. Marker aided selection for wood quality. In: Timber management toward wood quality and end-product value. CTIA/IUFRO International Wood Quality Workshop. Aug. 18-22, 1997. VI:11-17. Quebec City, Quebec, Canada.

Verhagen, D., Kremer, A., and Vigneron, Ph. 1995. In: Eucalypt plantations: Improving fibre yield and quality. Proc. CRC-IUFRO Conf., 19-24 Feb. pp. 434-437. Potts, B. M., Borralho, N. M. G., Reid, J. B., Cromer, R. N., Tibbits, W. N., and Raymond, C. A., Eds. CRC for Temperate Hardwood Forestry. Hobart, Australia.

Voo, K. S., Whetten, R. W., O’Malley, D. M., and Sederoff, R. R. 1995. 4-coumarate: coenzyme A ligase from loblolly pine xylem. Isolation, characterization, and complementary DNA cloning. Plant Physiol. 108(1):85-97.

Williams, C. G. and Neale, D. B. 1992. Conifer wood quality and marker-aided selection: A case study. Can. J. For. Res. 22:1009-1017.

Wooley, R., Ruth, M., Sheehan, J., and Ibsen, K. 1999. Lignocellulosic biomass to ethanol process design and economics utilizing co-current dilute acid prehydrolysis and enzymatic hydrolysis current and futuristic scenarios. US Dept. of Energy, National Renewable Energy Laboratory Tech. Rpt. NREL/TP-580-26157.

Wright, J. A., Birkett, M. D., and Gambino, M. J. T. 1990. Prediction of pulp yield and cellulose content from wood samples using near infrared reflectance spectroscopy. TAPPI J. 73(3):164-166.

Wright, L. L. and Tuskan, G. A. 1997. Strategy, results, and directions for woody crop research funded by the US Department of Energy. In: Proc. 1997 TAPPI Pulping Conf. pp. 791-799. TAPPI Press, Atlanta, GA.

Wyman, C. E. 1993. An overview of ethanol production for transportation fuels. In: Proc. of the First Biomass Conf. of the America: Energy, Environment, agriculture, and industry. August 30-September 2, 1993. pp. 1010-1031. Burlington, VT. 
Xue, B., Ellis, D., Newton, D., Gawley, B., Gibbert, M., and Sutton, B. 1997. Alternating carbon allocation to increase cellulose biosynthesis and plant production. Plant Physiol. 114(suppl 3):300.

Yanchuck, A. D., Dancik, B. P., and Micko, M. M. 1983. Intraclonal variation in wood density of trembling aspen in Alberta. Wood and Fiber Sci. 15(4):387-394.

Zhang, X. H. and Chiang, V. L. 1997. Molecular cloning of 4-coumarate:coenzyme A ligase in loblolly pine and the roles of this enzyme in the biosynthesis of lignin in compression wood. Plant Physiol. 113(1):65-74.

Ziegler, M. ., Thomas, S., and Danna, K. J. In press. Accumulation of a thermostable endo-1,4- $\beta$ D glucanase in the aopoplast of Arabidopsis thaliana leaves. Molecular Breeding.

Zobel, B. J., Campinhos, Jr., E., and Ikemori, Y. 1983. Selecting and breeding for desirable wood. TAPPI J. 66(1):70-74.

Zobel, B. J. and Jett, J. B. 1995. Genetics of wood production. Springer-Verlag, Berlin, Germany.

Zobel, B. J. and van Buijtenen, J. P. 1989. Wood variation: Its causes and control. SpringerVerlag, Berlin, Germany. 
ORNL/Sub/99-4500007253/99

\section{INTERNAL DISTRIBUTION}

1-50. L. L. Wright

51. Central Research Library

52-54. ESD Library (3 copies)
54-55. Laboratory Records-OSTI (2 copies)

56. Laboratory Records-RC

\section{EXTERNAL DISTRIBUTION}

57. Tom Blush, Westvaco, Forest Science Laboratory, P.O. Box 1950, Summerville, SC 29484

58. Bruce Bongarten, University of Georgia, School of Forest Resources, Athens, GA 30602

59. Toby Bradshaw, University of Washington, Ctr. for Urban Horticulture GF-15, Seattle, WA 98195

60. Daniel Carraway, International Paper Company, 719 Southlands Road, Bainbridge, GA 31717

61. Clint Chapple, Purdue University, Department of Biochemistry, West Lafayette, IN 47907-1153

62. Vincent Chiang, Michigan Technological University, School of Forestry \& Wood Products, Houghton, MI 49931

63. Bob Conger, University of Tennessee, Dept. of Plant and Soil Science, Knoxville, TN 37901-1071

64. Jeff Dean, University of Georgia, School of Forest Resources, Athens, GA 30602

65. Gregory Dilworth, U.S. Department of Energy, Energy Biosciences, Germantown, MD 20876

66. Don Dimmell, Institute of Paper Science and Technology, 500 19th Street, N.W., Atlanta, GA 30318-7754

67-76. Ronald Dinus, 2490 Goshen Road, Bellingham, WA 98226-9556

77. David Ellis, Cellfor, Inc., 3650 Westbrook Mall, Vancouver, B.C. V6S 2L2, Canada

78. John Ferrell, U.S. Dept. of Energy, Office of Fuels Development, 1000 Independence Avenue, EE-31/MS:6B-025 FORS, Washington, DC 20585

79. Sam Foster, USDA Forest Service, Southern Forest Experiment Station, Alabama A\&M University, Box 1387, Normal, AL 35762

80. Peter Gary, Institute of Paper Science and Technology, 500 10th Street, N.W., Atlanta, GA 30318-9754

81. David Glassner, 15305 Minetonka Blvd., Minetonka, MN 53345

82. Dario Grattapaglia, Laboratorio de Genetica de Plantas, EMBRAPA Recursos Geneticos e Biotecnologia, SAIN Parque Rural CP 02372, Basilia 70770-900, Brazil

83. Richard Hall, Iowa State University, Forestry Dept., 251 Bessey Hall, Ames, IA 50011

84. James Hettenhaus, Chief Executive Assistance, 3211 Trefoil Drive, Charlotte, NC 28226 
85. Thomas Jeffries, University of Wisconsin, Forest Products Laboratory, One Gifford Pinchot Drive, Madison, WI 53705

86. Randy Johnson, Forestry Sciences Laboratory, 3200 Jefferson Way, Corvallis, OR 97331-4401

87. Hans Jung, University of Minnesota, USDA-ARS, 411 Borlaug Hall, 1991

Upper Buford Circle, St Paul, MN 55108

88. Peter Keeling, ExSeed Genetics, L.L.C., Food Science Building, Iowa State University, Ames, IA 50011-1061

89. R. Kellison, Champion International, 1316 Dixie Trail, Raleigh NC 27607

90. Sam Land, Jr., Mississippi State University, Dept. of Forestry, P.O. Drawer FR 900, America's Center Bldg., Mississippi State, MS 39762

91. Carol Loopstra, Texas A\&M University, Mail Stop 2135, College Station, TX 77843

92. William Lowe, Texas Forest Service, Forest Science Lab, College Station, TX 77843

93. Ruth Mark, National Renewable Energy Laboratory, 1617 Cole Blvd., Golden, CO 80401

94. Scott Merkle, University of Georgia, School of Forest Resources, Athens, GA 30602

95. Charles H. Michler, Purdue University, Department of Forestry, West Lafayette, IN 47907

96. David Neale, Institute of Forest Genetics, Environmental Horticulture, University of California, Davis, CA 95616

97. Dana Nelson, International Paper Company, 719 Southlands Road, Bainbridge, GA 31717

98. Bob Northey, University of Washington, Department of Forest Resources, Box 352100, Seattle, WA 98195

99. Peggy Payne, Boise Cascade Fiber Farm, P.O. Box 500, Highway 12, Wallula, WA 99363

100. Gary Peters, IPST, 500 10th Street, N.W., Atlanta, GA 30318

101. Gilles Pilate, Station d'Amelioration des Arbres Forestiers, Institut National de la Recherches Agronomique (INRA), F-45160 Ardon, France

102. Robert Purnell, International Paper, Route 1, Box 421, Bainbridge GA 31717

103. John Ralph, USDA-ARS, 1925 Linden, Madison, WI 53706

104. Glen Rasmussen, E13273 E. Tower Road, Ringle, WI 54471

105. Don Rockwood, University of Florida, School of Forest Res. \& Conservation, 118 Newins-Ziegler Hall, Gainesville, FL 32611

106. Randy Rousseau, Westvaco, Central Forest Research, P.O. Box 458, Wickliffe, KY 42087

107. Rod Savidge, University of New Brunswick, P. O. Box 44555, Fredericton, New Brunswick E3B 6C2, Canada

108. Ron Sederoff, North Carolina State University, Dept. of Forestry, 6113 Jordan Hall, Box 8008, Raleigh, NC 27695-8008

109. $\quad$ Mitchell Sewell, 910 Woodvine Road, Asheville, NC 28803

110. Vincent Sewalt, Pioneer Hybrid International, 7300 N.W. 62nd Avenue, Johnston, IA 50131 
111. Sarah Sprague, U.S. Dept. of Energy, Office of Fuels Development, EE-31, 1000 Independence Avenue, Washington, DC 20585

112. Brian Stanton, James River Corp., Northwest Fiber Supply Division, 349 N.W. 7th Ave., Camas, WA 98607

113. Reinhard Stettler, 2133 E. Shelby St., Seattle, WA 98112

114. Michael Stine, Louisiana State University, School of Forestory, Baton Rouge, LA 70803

115. Steve Strauss, Oregon State University, Dept. of Forest Science, FSL 020, Corvallis OR 97331-7501

116. Ben Sutton, Cellfor, Inc., 3650 Westbrook Mall, Vancouver, B.C. V6S 2L2, Canada

117. Steven Thomas, National Renewable Energy Laboratory, 1617 Cole Blvd., Golden, CO 80401

118. Hans Van Buitjtenen, Texas Forest Service, Texas A\&M University ,Forest Genetics Lab, College Station, TX 77843

119. Ken Vogel, University of Nebraska, USDA-ARS, 344 Keim Hall, P.O. Box 830937, Lincoln, NE 68583-0937 
Lynn L. Wright (50 copies)

1059, MS-6422

ESD Library (3 copies)

1505, MS-6035

Laboratory Records-RC

$4500 N$, MS-6285

Bruce Bongarten

University of Georgia

School of Forest Resources

Athens, GA 30602

Daniel Carraway

International Paper Company

719 Southlands Road

Bainbridge, GA 31717
Central Research Library

4500N, MS-6191

Laboratory Records-OSTI ( 2 copies)

$4500 \mathrm{~N}, \mathrm{MS}-6285$

Tom Blush

Westvaco

Forest Science Laboratory

P.O. Box 1950

Summerville, SC 29484

Toby Bradshaw

University of Washington

Ctr. for Urban Horticulture GF-15

Seattle, WA 98195

Clint Chapple

Purdue University

Department of Biochemistry

West Lafayette, IN 47907-1153 
Vincent Chiang

Michigan Technological University

School of Forestry \& Wood Products

Houghton, MI 49931

Jeff Dean

University of Georgia

School of Forest Resources

Athens, GA 30602

Don Dimmell

Institute of Paper Science and Technology 500 19th Street, N.W.

Atlanta, GA 30318-7754

David Ellis

Cellfor, Inc.

3650 Westbrook Mall

Vancouver, B.C.

V6S 2L2, Canada

Sam Foster

USDA Forest Service

Southern Forest Experiment Station

Alabama A\&M University

Box 1387

Normal, AL 35762
Bob Conger

University of Tennessee

Dept. of Plant and Soil Science

Knoxville, TN 37901-1071

Gregory Dilworth

U.S. Department of Energy

Energy Biosciences

Germantown, MD 20876

Ronald Dinus

2490 Goshen Road

Bellingham, WA 98226-9556

John Ferrell

U.S. Dept. of Energy

Office of Fuels Development

1000 Independence Avenue

EE-31/MS:6B-025 FORS

Washington, DC 20585

Peter Gary

Institute of Paper Science and Technology

500 10th Street, N.W.

Atlanta, GA 30318-9754 
David Glassner

15305 Minetonka Blvd.

Minetonka, MN 53345

Richard Hall

Iowa State University, Forestry Dept.

251 Bessey Hall

Ames, IA 50011

Thomas Jeffries

University of Wisconsin

Forest Products Laboratory

One Gifford Pinchot Drive

Madison, WI 53705

Hans Jung

University of Minnesota

USDA-ARS

411 Borlaug Hall

1991 Upper Buford Circle

St Paul, MN 55108

R. Kellison

Champion International

1316 Dixie Trail

Raleigh NC 27607
Dario Grattapaglia

Laboratorio de Genetica de Plantas

EMBRAPA Recursos Geneticos e Biotecnologia

SAIN Parque Rural CP 02372

Basilia 70770-900, Brazil

James Hettenhaus

Chief Executive Assistance

3211 Trefoil Drive

Charlotte, NC 28226

Randy Johnson

Forestry Sciences Laboratory

3200 Jefferson Way

Corvallis, OR 97331-4401
Peter Keeling

ExSeed Genetics, L.L.C.

Food Science Building

Iowa State University

Ames, IA 50011-1061
Sam Land, Jr.

Mississippi State University

Dept. of Forestry

P.O. Drawer FR 900

America's Center Bldg.

Mississippi State, MS 39762 
Carol Loopstra

Texas A\&M University

Mail Stop 2135

College Station, TX 77843
William Lowe

Texas Forest Service

Forest Science Lab

College Station, TX 77843
Ruth Mark

National Renewable Energy Laboratory 1617 Cole Blvd.

Golden, CO 80401
Scott Merkle

University of Georgia

School of Forest Resources

Athens, GA 30602

David Neale

Institute of Forest Genetics

Environmental Horticulture

University of California

Davis, CA 95616
Dana Nelson

International Paper Company

719 Southlands Road

Bainbridge, GA 31717
Bob Northey

University of Washington

Department of Forest Resources

Box 352100

Seattle, WA 98195
Peggy Payne

Boise Cascade Fiber Farm

P.O. Box 500, Highway 12

Wallula, WA 99363
Gary Peters

IPST

500 10th Street, N.W.

Atlanta, GA 30318 
Gilles Pilate

Station d'Amelioration des Arbres Forestiers

Institut National de la Recherches Agronomique (INRA)

F-45160 Ardon, France
Robert Purnell

International Paper

Route 1, Box 421

Bainbridge GA 31717
John Ralph

USDA-ARS

1925 Linden

Madison, WI 53706
Glen Rasmussen

E13273 E. Tower Road

Ringle, WI 54471
Don Rockwood

University of Florida

School of Forest Res. \& Conservation

118 Newins-Ziegler Hall

Gainesville, FL 32611
Randy Rousseau

Westvaco

Central Forest Research

P.O. Box 458

Wickliffe, KY 42087
Rod Savidge

University of New Brunswick

P. O. Box 44555

Fredericton, New Brunswick

E3B 6C2, Canada
Ron Sederoff

North Carolina State University

Dept. of Forestry

6113 Jordan Hall, Box 8008

Raleigh, NC 27695-8008
Mitchell Sewell

910 Woodvine Road

Asheville, NC 28803
Vincent Sewalt

Pioneer Hybrid International

7300 N.W. 62nd Avenue

Johnston ,IA 50131 
Sarah Sprague

U.S. Dept. of Energy

Office of Fuels Development, EE-31

1000 Independence Avenue, Washington, DC 20585

Reinhard Stettler

2133 E. Shelby St.

Seattle, WA 98112

Steve Strauss

Oregon State University

Dept. of Forest Science, FSL 020

Corvallis OR 97331-7501

Steven Thomas

National Renewable Energy Laboratory

1617 Cole Blvd.

Golden, CO 80401
Brian Stanton

James River Corp.

Northwest Fiber Supply Division

349 N.W. 7th Ave.

Camas, WA 98607

Michael Stine

Louisiana State University

School of Forestory

Baton Rouge, LA 70803
Ben Sutton

Cellfor, Inc.

3650 Westbrook Mall

Vancouver, B.C.

V6S 2L2, Canada
Hans Van Buitjtenen

Texas Forest Service

Texas A\&M University

Forest Genetics Lab

College Station, TX 77843

Ken Vogel

University of Nebraska

USDA-ARS

344 Keim Hall

P.O. Box 830937

Lincoln, NE 68583-0937 